University of Tennessee Health Science Center UTHSC Digital Commons

\title{
Factors Influencing Topotecan CNS Penetration in Mouse Models
}

Jun Shen

University of Tennessee Health Science Center

Follow this and additional works at: https://dc.uthsc.edu/dissertations

Part of the Neurosciences Commons

\section{Recommended Citation}

Shen, Jun , "Factors Influencing Topotecan CNS Penetration in Mouse Models" (2008). Theses and Dissertations (ETD). Paper 241. http://dx.doi.org/10.21007/etd.cghs.2008.0288.

This Dissertation is brought to you for free and open access by the College of Graduate Health Sciences at UTHSC Digital Commons. It has been accepted for inclusion in Theses and Dissertations (ETD) by an authorized administrator of UTHSC Digital Commons. For more information, please contact jwelch30@uthsc.edu. 


\title{
Factors Influencing Topotecan CNS Penetration in Mouse Models
}

\begin{abstract}
Camptothecin analogs such as topotecan are currently tested in clinical trials for brain tumors. However the clinical outcome is far below the expectations, which are derived from the promising preclinical studies. This discrepancy could be partially attributed to the presence of two barrier systems in the brain, the blood brain barrier (BBB) and blood cerebrospinal fluid (CSF) barrier (BCB). Further investigations have demonstrated multiple ATP-binding cassette $(A B C)$ transporters are present at the two barriers. Camptothecin analogs are well known substrates for several of the transporters. This work defined the role of two ABC transporters, Bcrp1 and P-gp in determination of topotecan CNS penetration in mouse models by using cerebral microdialysis. Microdialysis sampling of drugs in ventricular cerebrospinal fluid (vCSF) provides insight into drug penetration into the brain across the blood CSF barrier (BCB); however, this method has been reported primarily in larger animal species. The initial studies were designed to improve our existing surgical technique to implant a microdialysis cannula into the lateral ventricle to sample vCSF. The modifications done in these studies consisted of changes in the stereotaxic coordinates and insertion of the cannula at a $20^{\circ}$ angle. Exact placement of the probe was confirmed using ultrasound (US), micro-computed tomography (CT), and histologic review of serial paraffin sections. Studies of topotecan CSF penetration in the FVB mouse using this modified approach determined that the VCSF to plasma AUC ratio of unbound topotecan lactone was greater than that previously reported using previous surgical technique. Results of studies from this lab have shown that topotecan has differential CNS penetration, and that VCSF topotecan penetration is greater than penetration into brain parenchymal extracellular fluid (ECF). We hypothesize Bcrp1 and P-gp together play a major role in regulation of topotecan CNS penetration. To further characterize the topotecan CNS transport mechanisms, topotecan penetration in two CNS compartments, vCSF and brain parenchymal ECF were determined in several knockout mouse models (Bcrp1(-/-), Mdr1a/b(-/-) and Bcrp1/Mdr1a/b(-/-) by adapting an intracerebral microdialysis technique. After an intravenous (i.v.) bolus dose of $4 \mathrm{mg} / \mathrm{kg}$ topotecan, unbound topotecan lactone vCSF penetration was defined by a vCSF to plasma area under the curve ratio (AUCu,vCSF/ AUCu,plasma). The topotecan vCSF penetration (AUCu,ECF/AUCu,plasma) was significantly decreased in all three knockout models compared with wild type. The penetration into the brain parenchyma, defined by the ECF to plasma AUC ratio, was not changed in Bcrp1(-/-) but significantly increased in Bcrp1/Mdr1a/b(/-). Pre-treatment with the EGFR tyrosine kinase inhibitor gefitinib $(200 \mathrm{mg} / \mathrm{kg})$ to inhibit Bcrp1 and P-gp transporters significantly decreased topotecan VCSF penetration but increased ECF penetration in FVB wild type. The topotecan lactone to carboxylate AUC ratio in vCSF (AUClactone,CSF/AUCcarboxylate,CSF) was calculated in FVB wild type and three knockout models. Interestingly, a preferential disposition of topotecan lactone over carboxylate was apparent in vCSF of wild type, Bcrp1(-/-) and Mdr1a/b(-/-) but not in Bcrp1/Mdr1a/b(-/-). These studies are the first to carefully assess the contribution of efflux transporters to topotecan penetration into brain ECF and vCSF, and to assess the importance of lactone compared with carboxylate. Whereas these studies have all been conducted in a non-tumor bearing model, we evaluated the effect of the presence of a primary CNS tumor on topotecan CNS penetration. An orthotopic human glioblastoma (luciferase labeled U-87) mouse model was constructed. Preliminary intratumoral microdialysis studies have not found different topotecan disposition in tumor from normal brain. However, the model might be biased due to the position of tumor inoculation and will require further study for clarification. In conclusion, our findings suggest ABC transporters, Bcrp1 and P-gp, play a major role in topotecan CNS penetration by enhancing topotecan penetration into vCSF and restricting penetration into ECF. This insight can be translated in clinical studies to improve topotecan CNS penetration.
\end{abstract}

Document Type

Dissertation 


\section{Degree Name}

Doctor of Philosophy (PhD)

\section{Program}

Interdisciplinary Program

\section{Research Advisor}

Clinton Stewart, Pharm.D.

\section{Keywords}

Blood brain barrier, Blood CSF barrier, CNS, Topotecan, Pharmacokinetics, ABC-transporters, Microdialysis, Mouse tumor model

\section{Subject Categories}

Medical Sciences | Medicine and Health Sciences | Neurosciences 


\title{
FACTORS INFLUENCING TOPOTECAN CNS PENETRATION IN MOUSE MODELS
}

\author{
A Dissertation \\ Presented for \\ The Graduate Studies Council \\ The University of Tennessee \\ Health Science Center
}

In Partial Fulfillment

Of the Requirements for the Degree

Doctor of Philosophy

From The University of Tennessee

By

Jun Shen

May 2008 
Chapter 2 (C) 2008 by John Wiley \& Sons, Inc.

All other material (C) 2008 by Jun Shen. 


\section{Acknowledgements}

I would like to express my appreciation to my mentor Dr. Clinton F. Stewart for his constant guidance, encouragement and inspiration. My sincere gratitude is also extended to my committee members, Dr. Christopher M. Waters, Dr. Alex Sparreboom, Dr. Bernd Meibohm, and Dr. Shannon G. Matta for their support, constructive opinions and suggestions through this project.

I would also like to extend my appreciation to the lab supervisors Charles Fraga, Laura Miller, and co-workers Dr. Yanli Zhuang, Dr. Feng Bai, Dr. Jiaowang Dong, Dr. John C. Panetta, Dr. Stacy Throm, Dr. Paula Schaiquevich, K. Elaine Hubbard, Henry G. Wynn, Fan Zhang, Thandranese Owens, Dr. Angel M. Carcaboso and Dr. Christopher Calabrese, Melissa Johnson, John Killmar, Dr. Kelli Body, June Bursi, Dr. Richard J. Rahija from Animal Resources Center, Dr. Thomas Sims from Department of Surgery and Dr. M. Beth McCarville from Department of Diagonostic Imaging for their help through the project. 


\begin{abstract}
Camptothecin analogs such as topotecan are currently tested in clinical trials for brain tumors. However the clinical outcome is far below the expectations, which are derived from the promising preclinical studies. This discrepancy could be partially attributed to the presence of two barrier systems in the brain, the blood brain barrier (BBB) and blood cerebrospinal fluid (CSF) barrier (BCB). Further investigations have demonstrated multiple ATP-binding cassette $(\mathrm{ABC})$ transporters are present at the two barriers. Camptothecin analogs are well known substrates for several of the transporters. This work defined the role of two ABC transporters, Bcrp1 and P-gp in determination of topotecan CNS penetration in mouse models by using cerebral microdialysis.

Microdialysis sampling of drugs in ventricular cerebrospinal fluid (vCSF) provides insight into drug penetration into the brain across the blood CSF barrier (BCB); however, this method has been reported primarily in larger animal species. The initial studies were designed to improve our existing surgical technique to implant a microdialysis cannula into the lateral ventricle to sample vCSF. The modifications done in these studies consisted of changes in the stereotaxic coordinates and insertion of the cannula at a $20^{\circ}$ angle. Exact placement of the probe was confirmed using ultrasound (US), micro-computed tomography (CT), and histologic review of serial paraffin sections. Studies of topotecan CSF penetration in the FVB mouse using this modified approach determined that the $\mathrm{vCSF}$ to plasma AUC ratio of unbound topotecan lactone was greater than that previously reported using previous surgical technique.
\end{abstract}

Results of studies from this lab have shown that topotecan has differential CNS penetration, and that vCSF topotecan penetration is greater than penetration into brain parenchymal extracellular fluid (ECF). We hypothesize Bcrp1 and P-gp together play a major role in regulation of topotecan CNS penetration. To further characterize the topotecan CNS transport mechanisms, topotecan penetration in two CNS compartments, vCSF and brain parenchymal ECF were determined in several knockout mouse models $\left(\right.$ Bcrp $^{(-/)}, M d r l a / b^{(--)}$and Bcrp $\left.1 / M d r l a / b^{(-/)}\right)$by adapting an intracerebral microdialysis technique. After an intravenous (i.v.) bolus dose of $4 \mathrm{mg} / \mathrm{kg}$ topotecan, unbound topotecan lactone vCSF penetration was defined by a vCSF to plasma area under the curve ratio $\left(\mathrm{AUC}_{\mathrm{u}, \mathrm{vCSF}} / \mathrm{AUC}_{\mathrm{u} \text {,plasma }}\right)$. The topotecan $\mathrm{vCSF}$ penetration $\left(\mathrm{AUC}_{\mathrm{u}, \mathrm{ECF}} / \mathrm{AUC}_{\mathrm{u} \text {,plasma }}\right)$ was significantly decreased in all three knockout models compared with wild type. The penetration into the brain parenchyma, defined by the ECF to plasma AUC ratio, was not changed in $\mathrm{Bcrp}^{(--)}$but significantly increased in Bcrp $1 / M d r l a / b^{(-/)}$. Pre-treatment with the EGFR tyrosine kinase inhibitor gefitinib (200 $\mathrm{mg} / \mathrm{kg}$ ) to inhibit Bcrp1 and P-gp transporters significantly decreased topotecan vCSF penetration but increased ECF penetration in FVB wild type. The topotecan lactone to carboxylate $\mathrm{AUC}$ ratio in $\mathrm{VCSF}\left(\mathrm{AUC}_{\text {lactone, } \mathrm{CSF}} / \mathrm{AUC}_{\text {carboxylate, } \mathrm{CSF}}\right.$ ) was calculated in $\mathrm{FVB}$ wild type and three knockout models. Interestingly, a preferential disposition of topotecan lactone over carboxylate was apparent in vCSF of wild type, $B \operatorname{crp}^{(-/)}$and Mdrla/b $/^{(-/)}$but not in Bcrpl/Mdrla/ $b^{(--)}$. These studies are the first to carefully assess the 
contribution of efflux transporters to topotecan penetration into brain ECF and vCSF, and to assess the importance of lactone compared with carboxylate.

Whereas these studies have all been conducted in a non-tumor bearing model, we evaluated the effect of the presence of a primary CNS tumor on topotecan CNS penetration. An orthotopic human glioblastoma (luciferase labeled U-87) mouse model was constructed. Preliminary intratumoral microdialysis studies have not found different topotecan disposition in tumor from normal brain. However, the model might be biased due to the position of tumor inoculation and will require further study for clarification.

In conclusion, our findings suggest $\mathrm{ABC}$ transporters, Bcrp1 and P-gp, play a major role in topotecan CNS penetration by enhancing topotecan penetration into vCSF and restricting penetration into ECF. This insight can be translated in clinical studies to improve topotecan CNS penetration. 


\section{Table of Contents}

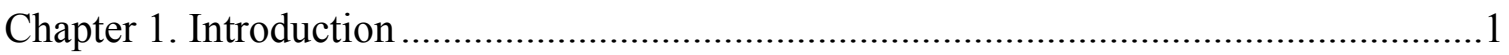

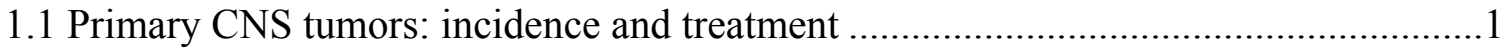

1.1.1 Incidence of primary CNS tumors ……………............................................

1.1.2 Treatment for primary CNS tumors in children...............................................

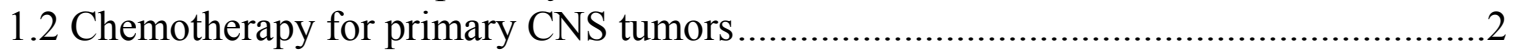

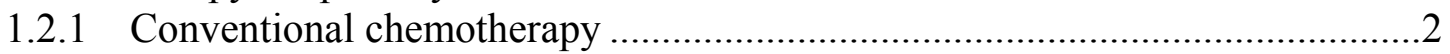

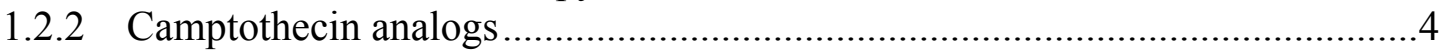

1.3 Blood brain barrier (BBB) and blood CSF barrier (BCB) .......................................

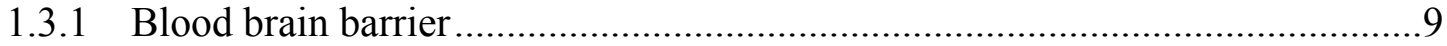

1.3.2 Blood cerebrospinal fluid barrier ............................................................11

1.4 The effect of $\mathrm{ABC}$ transporters on camptothecin analogs CNS penetration ...............12

1.4.1 The role of $\mathrm{ABC}$ transporters in vitro studies ..............................................14

1.4.2 The utilization of targeted mutation mouse models of $\mathrm{ABC}$ transporters .......15

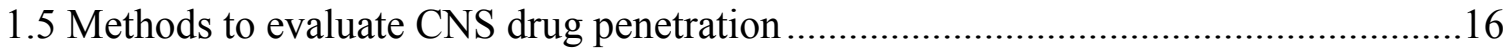

1.5.1 Homogenization and autoradiography method..............................................16

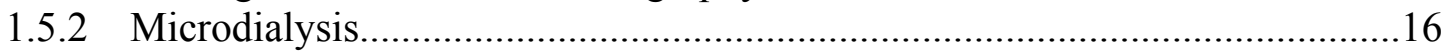

1.5.3 Pharmacokinetic considerations................................................................... 18

1.5.4 Modulation of $\mathrm{ABC}$ transporters by 4-anilinoquinazolines............................20

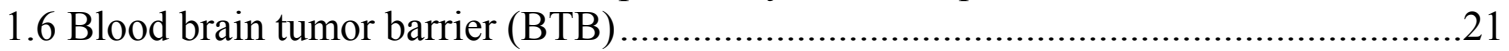

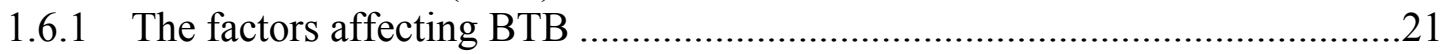

1.6.2 Early studies on drug penetration in the brain tumors ....................................24

1.6.3 In vivo microdialysis studies on drug penetration through BTB .....................25

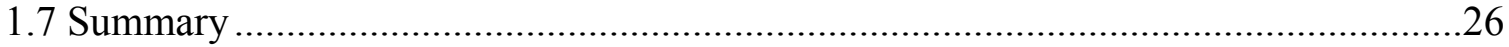

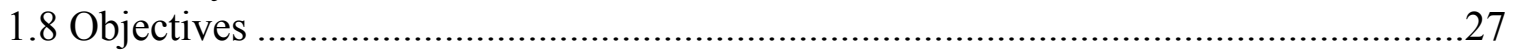

Chapter 2. A Modified Surgical Procedure for Microdialysis Probe Implantation in the Lateral Ventricle of an FVB Mouse....................................................28

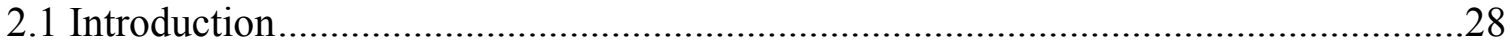

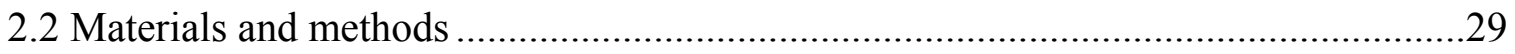

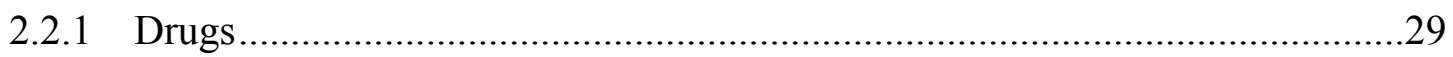

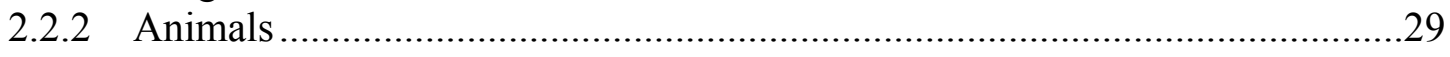

2.2.3 Stereotaxic coordinates calculation and adjustment ........................................29

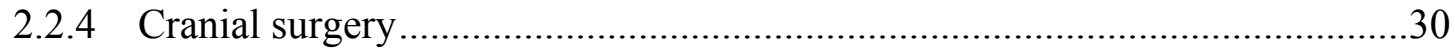

2.2.5 Ultrasound imaging....................................................................................

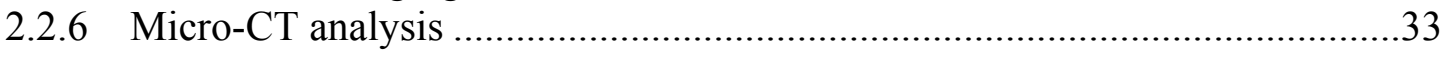

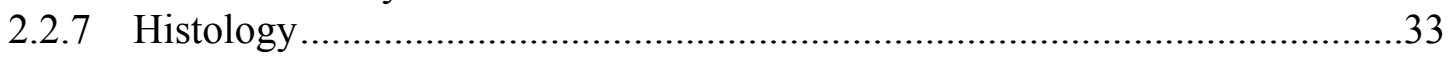

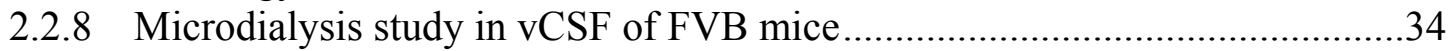

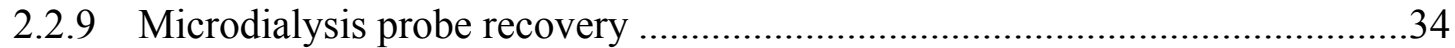

2.2.10 Noncompartmental analysis of vCSF pharmacokinetic data .............................34

2.2.11 Compartmental analysis of plasma pharmacokinetic data.................................35

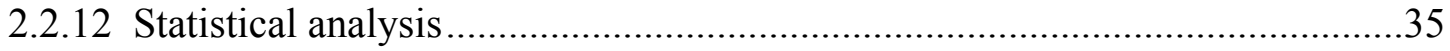




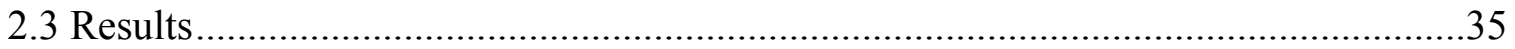

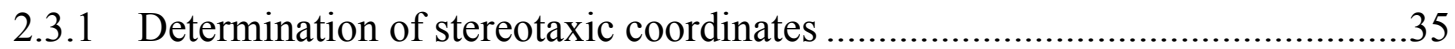

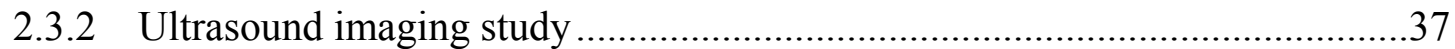

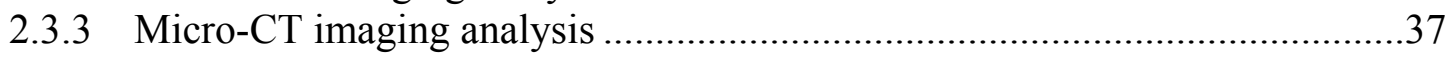

2.3.4 Topotecan penetration through blood CSF barrier in FVB mice.......................37

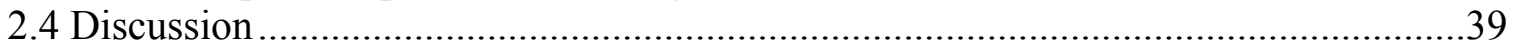

Chapter 3. Penetration of Topotecan Lactone across Blood-Brain Barrier and Blood-

CSF Barrier: Differential Role of P-Glycoprotein (P-gp) and Breast

Cancer Resistance Protein (BCRP) ............................................................43

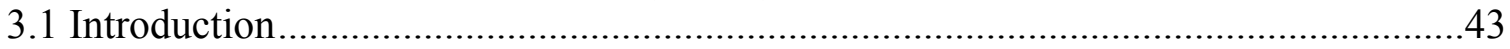

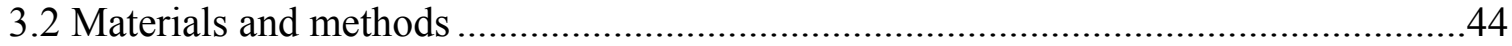

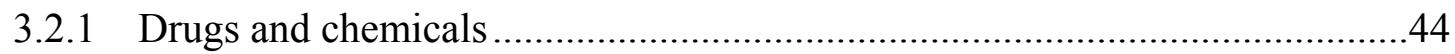

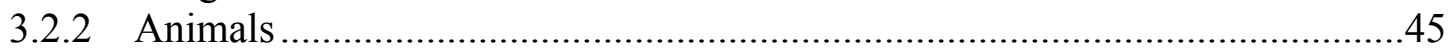

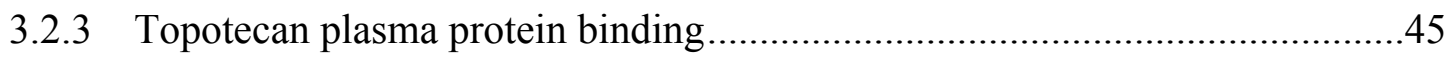

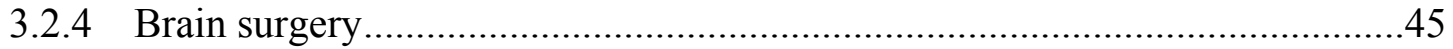

3.2.5 Microdialysis in ventricular CSF and brain ECF ........................................46

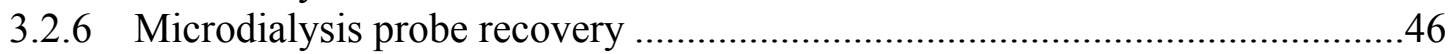

3.2.7 Noncompartmental analysis of brain topotecan data ........................................46

3.2.8 Topotecan plasma pharmacokinetics ……………….....................................4

3.2.9 Three-compartment analysis of combined plasma and brain lactone

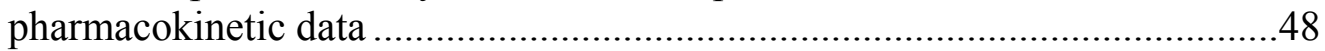

3.2.10 Three-compartment analysis of plasma topotecan lactone and carboxylate

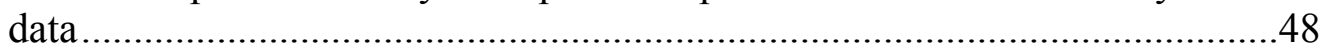

3.2.11 Immunohistochemistry ..........................................................................49

3.2.12 In vitro study of effect of $\mathrm{pH}$ on topotecan lactone and carboxylate

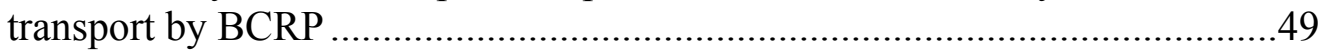

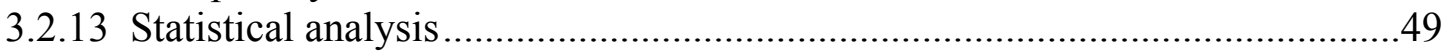

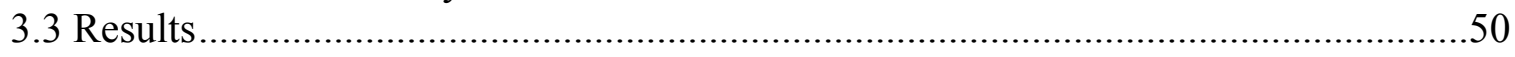

3.3.1 Topotecan lactone protein binding...........................................................50

3.3.2 Bcrp1 and P-gp enhance topotecan penetration into ventricular CSF .............50

3.3.3 Bcrp1 and P-gp decrease topotecan penetration into brain ECF .....................54

3.3.4 Differential regulatory effects of gefitinib on topotecan penetration into

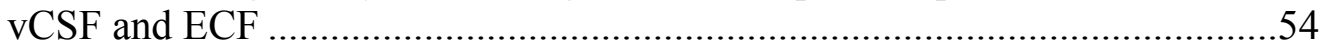

3.3.5 Preferential disposition of topotecan lactone in vCSF ....................................54

3.3.6 Saos2-BCRP cells preferentially transport topotecan lactone …………….......58

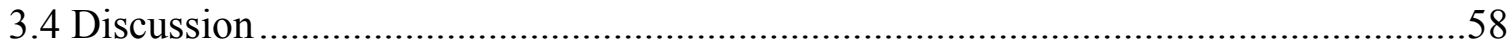

Chapter 4. Topotecan Penetration in an Intracranial Human Glioblastoma (U-87)

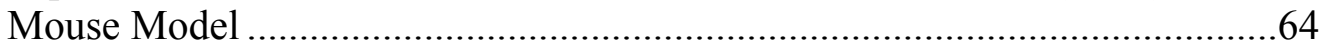

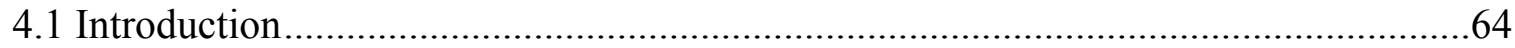

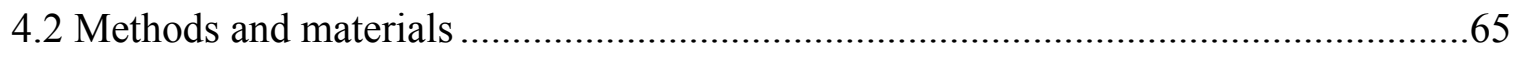

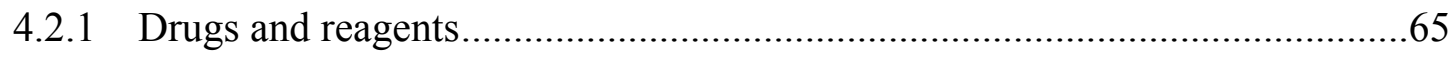

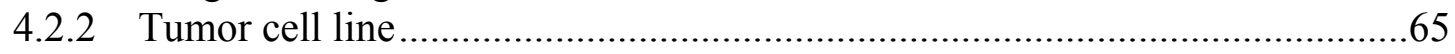

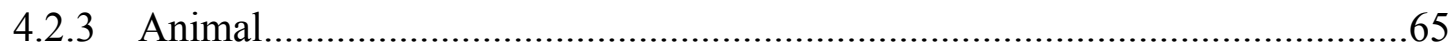

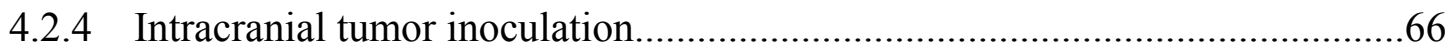


4.2.5 Microdialysis guide cannula implant .............................................................66

4.2.6 Bioluminescence imaging .........................................................................66

4.2.7 Correlation of tumor growth with bioluminescence signal..............................67

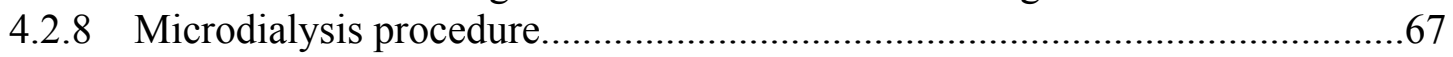

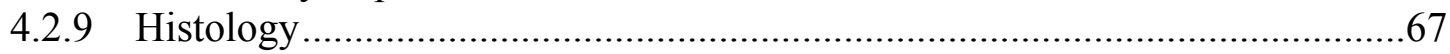

4.2.10 Noncompartmental analysis of brain pharmacokinetic data ............................68

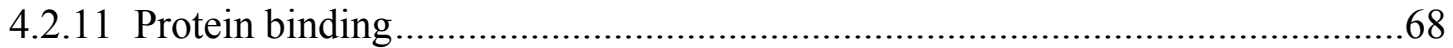

4.2.12 Compartmental analysis of plasma pharmacokinetic data...............................68

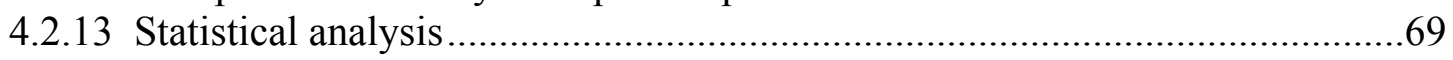

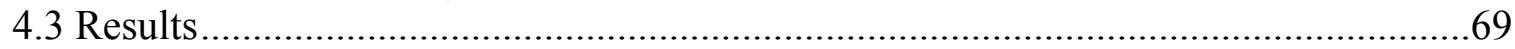

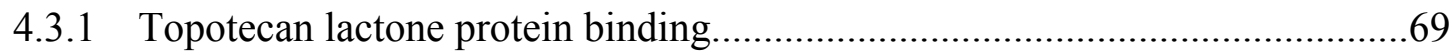

4.3.2 Tumor volume correlated to bioluminescence signal ......................................69

4.3.3 Topotecan penetration is not statistically different in the U-87 tumor tissue

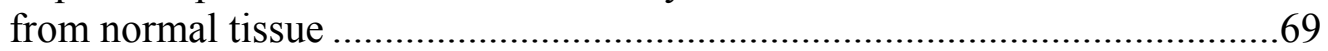

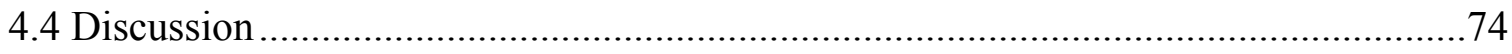

Chapter 5. Summary and Future Directions ……………..............................................

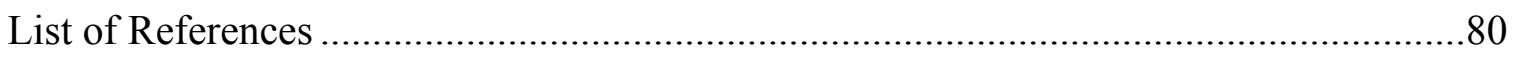

Appendix: Analysis of Topotecan Clearances across BCB or BBB ...............................103

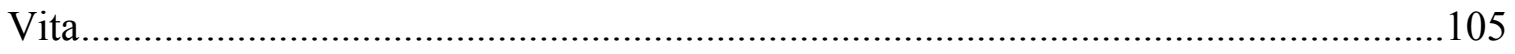




\section{List of Tables}

Table 2-1 vCSF to plasma AUC ratios of unbound topotecan lactone at the presence or absence of gefitinib.

Table 3-1 Noncompartmental (AUC ratio) and compartmental analysis (CLin/CLout) for brain (CSF or ECF) to plasma AUC ratio of unbound topotecan lactone in various mouse models..............................53 


\section{List of Figures}

Figure 1-1 Structures of camptothecin analogs. .................................................

Figure 1-2 Blood brain barrier................................................................. 10

Figure 1-3 Blood cerebrospinal fluid barrier. ......................................................13

Figure 1-4 General two-compartment model for drug transport between plasma and brain.

Figure 1-5 Blood endothelial cells in normal or tumor tissues...............................23

Figure 2-1 Schematic demonstration of sterotaxic coordinates calculation................31

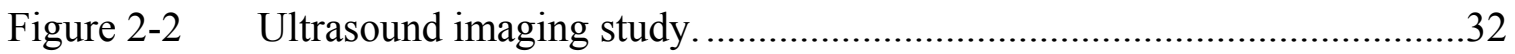

Figure 2-3 H\&E staining of sagittal brain section after probe insertion.....................36

Figure 2-4 MicroCT analysis of intact head and excised brain following MicroFil

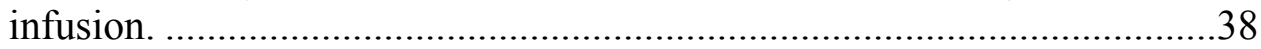

Figure 3-1 Microdialysis probe track in lateral ventricle or parenchymal tissue........51

Figure 3-2 Topotecan lactone penetration into vCSF in various mouse models at one dose of topotecan $4 \mathrm{mg} / \mathrm{kg}$ i.v. bolus.

Figure 3-3 Topotecan lactone penetration into brain ECF in various mouse models at one dose of topotecan $4 \mathrm{mg} / \mathrm{kg}$ i.v. bolus.

Figure 3-4 Regulatory effect of gefitinib on topotecan penetration into brain ECF or vCSF at one dose of topotecan $4 \mathrm{mg} / \mathrm{kg}$ i.v. bolus and gefitinib $200 \mathrm{mg} / \mathrm{kg}$ oral gavage pretreatment

Figure 3-5 vCSF lactone to carboxylate AUC ratio of unbound topotecan. ..............57

Figure 3-6 $\mathrm{pH}$ effect on topotecan lactone and carboxylate transport by BCRP........59

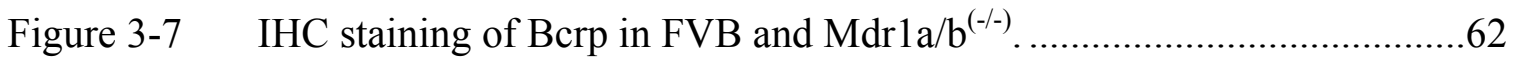

Figure 4-1 Tumor (U-87) growth monitored by bioluminescence ..........................70

Figure 4-2 Correlation of tumor volume to bioluminescence signal ........................71

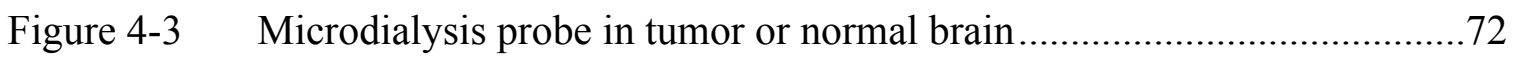


Figure 4-4 TPT penetration in the U-87 tumor model of CD-1 nude mice... 


\section{List of Abbreviations}

$\mathrm{ABC}$ transporters

Adenosine triphosphate-binding cassette transporters

aCSF

Artificial CSF

ATP

Adenosine triphosphate

AUC

Area under the concentration-time curve

$\mathrm{BBB}$

Blood-brain barrier

$\mathrm{BCB}$

Blood-cerebrospinal fluid barrier

BCRP

Breast cancer resistance protein

BMS-354825

Dasatinib, Sprycel

BTB

Blood-brain tumor barrier

CL ..Clearance

cLogP calculated $\log \mathrm{P}$

CNS Central nervous system

COG

CPTs

CSF ..Camptothecins

DMSO Cerebrospinal fluid

ECF Dimethyl sulfoxide

EDT Extracellular fluid

EGFR/Erbb1

Exposure duration threshold

GF120918

GW572016.

IFP Children's Oncology Group

ILs Epidermal growth factor receptor

IRN

$\log \mathrm{P}$ Epidermal growth factor receptor

LTC4

MMPs

MRP Lapatinib, Tykerb Interstitial fluid pressure

MTT Interleukins

NONMEM

OATs Iirinotecan, CPT-11, Camptosar Leukotriene $\mathrm{C} 4$

OATPs

OCTs

OS Matrix metalloproteinases Multidrug resistance protein

OSI-774

PBS

PFS

P-gp

PNET.

POG.

PSC833

RTKs

SLC

4, 5-Dimethylthiazol-2-yl]-2, 5-diphenyl tetrazolium bromide

Nonlinear mixed-effect modeling Organic anion transporters Organic anion transporting polypeptides Organic cation transporters Overall survival Erlotinib, Tarceva Phosphate-buffered saline .Progression free survival ..P-glycoprotein, MDR1 Primitive neuroectodermal tumors Pediatric Oncology Group Valspodar Receptor tyrosine kinases Solute carrier family 
STI-571

Imatinib, Gleevec TGF- $\beta$

TJ. Transforming growth factor- $\beta$

TK Tight junction

TKI Tyrosine kinase TPT TPT C.

TPT L

vCSF VEGF ZD1839

ZD6474

4-AQ Tyrosine kinase inhibitor Topotecan carboxylate Topotecan lactone . Ventricular cerebrospinal fluid .Vascular endothelial growth factor ..Gefitinib, Iressa Vandetanib, Zactima 4-aninlinoquinazoline 


\section{Chapter 1. Introduction}

\subsection{Primary CNS tumors: incidence and treatment}

\subsubsection{Incidence of primary CNS tumors}

The American Cancer Society estimates 20,500 new cases of primary brain and other nervous system tumors will be diagnosed in the United States in 2007. They also estimate that over 12,000 patients will die from these tumors (Jemal et al., 2007). Central nervous system (CNS) tumors are the most common solid malignancy in children with an estimated 3,200 children diagnosed with primary CNS tumors in 2007(American Cancer Society, 2007). According to a recent report by the Centers for Disease Control and Prevention (CDC), CNS tumors were the second leading cause of death in persons aged 1-19 years in the United States accounting for 25\% of total cancer deaths (Centers for Disease Control and Prevention, 2007). In the United States, although the annual mortality rate of pediatric cancers has steadily decreased over the past two decades, the proportion of deaths from CNS tumors in the same population has increased from $18 \%$ to $30 \%$ (Gururangan and Friedman, 2004). Worldwide, approximately 30,000-40,000 children develop CNS tumors each year, and the majority of them do not survive despite aggressive therapy (Bleyer, 1999; Fisher et al., 2007).

CNS tumors are classified mainly by location and histopathology into the following groups: Gliomas (including astrocytoma (grade II), anaplastic astrocytoma (grade III), glioblastoma (grade IV) and other gliomas), primitive neuroectodermal tumors (PNETs), medulloblastoma, meningioma, germ cell tumors and tumors at sellar region (including pituitary tumors and craniopharyngioma) (Fisher et al., 2007). In adults gliomas are the most common CNS tumors in which glioblastoma multiforme (GBM), the most malignant glioma, is the primary histological type. Other frequent adult CNS tumors include meningiomas and brain metastasis mainly from lung and breast cancers (Nguyen and DeAngelis, 2007).

In children, low grade astrocytomas are the most common CNS tumors comprising more than $50 \%$ of total pediatric CNS tumors then followed by medulloblastoma (16\%) and glioblastoma (7\%) (Ullrich and Pomeroy, 2003; Gururangan and Friedman, 2004). Originating from neuroepithelial tissue, most gliomas are located in brain parenchymal tissue and are perfused by capillaries in the brain. However medulloblastoma, now recognized as a molecularly distinct tumor from PNET, is located in cerebellum and has direct access to cerebrospinal fluid (CSF) (Pomeroy et al., 2002). Tumor location, along with other properties (e.g. tumor size, tumor genetics), serve as important prognostic factors (Fisher et al., 2007) as well as treatment option considerations. 


\subsubsection{Treatment for primary CNS tumors in children}

Currently three treatment modalities are available for pediatric CNS tumors, surgical resection, radiation therapy, and chemotherapy. In most cases, surgical resection is the first option. Surgery alone or combined with radiation therapy may cure many tumors, including some cerebellar astrocytomas, ependymomas, meningiomas, and some low-grade gliomas (Stieber and Mehta, 2007; Asthagiri et al., 2007; Young, 2007). Children with high grade tumors, such as anaplastic astrocytomas are not cured by surgery alone. Surgery however will reduce the amount of tumor that remains to be treated by radiation or chemotherapy. Moreover patients with diseases with specific histopathological properties (e.g., infiltrating gliomas mixed with critical cortical functional regions) cannot be treated with surgery and require other therapies (Asthagiri et al., 2007). Radiation therapy also plays a primary role in management of most malignant and many benign CNS tumors (Stieber and Mehta, 2007). Since high doses of radiation can damage normal brain tissue, techniques (e.g., stereotaxic radiosurgery) have been developed to precisely target tumor tissues and spare normal tissues. The exception to this is patients younger than 3 years of age. Because the brain of a child is still growing, radiation may severely impair neurocognitive capabilities. Although rare, radiation may cause DNA damage which may lead to a secondary malignancy (e.g., meningiomas) (Stieber and Mehta, 2007; Al-Mefty et al., 2004). Chemotherapy is generally used for high-grade tumors and may be given before or after surgery and radiation therapy. The treatment for children under 3 years old usually relies mainly on chemotherapy after surgery (American Cancer Society, 2007). The goal of chemotherapy is to further prolong survival after surgery and radiation, and maintain the quality of life(Castro et al., 2003).

\subsection{Chemotherapy for primary CNS tumors}

\subsubsection{Conventional chemotherapy}

Drug treatment for brain tumors in children and adults began to take form in the early 1970s, when clinical benefits were observed from nitrosoureas in adult malignant gliomas (Finlay, 1999). At the same time, vincristine and nitrosoureas were also reported to have activity in recurrent childhood brain tumors (Finlay, 1999). When radiotherapy had been recognized to cause irreversible damage to the brain of young children, chemotherapy became an important treatment option. Conventional chemotherapy usually included multiple drugs from nitrosoureas (e.g., lomustine), mitotic spindle inhibitors (e.g., vincristine), alkylating agents (e.g., melphalan), platinum complexes (e.g., cisplatin), antimetabolites (e.g., methotrexate) and glucocorticoids (e.g., dexamethasone). Different combinations of these types of drugs were tested in clinical trials. In addition to these conventional agents, drugs with novel mechanisms (e.g., camptothecin analogs, temozolomide, and $\mathrm{O}^{6}$-benzylguanine etc.) have been introduced to adjuvant chemotherapies (Broniscer et al., 2007; Lamers et al., 2008; Stewart et al., 2004a). 
Medulloblastoma is the most common malignant pediatric solid tumor. Based on tumor size and location, medulloblastoma can be classified in stages with increasing risk from T1 to T4. Alternatively it can also be classified from M0 to M4 with increasing risk of metastasis. Standard therapy for children with medulloblastoma consists of maximal surgical resection followed by craniospinal irradiation combined with adjuvant chemotherapy. Early clinical studies were conducted by multiple groups, including Children's Cancer Group (CCG) (Evans et al., 1990), International Society of Pediatric Oncology (SIOP) (Tait et al., 1990) and Pediatric Oncology Group (POG) (Krischer et al., 1991). The results were similar with a long term survival rates of approximately $55 \%$ at 5 years and $45 \%$ at 10 years. However the survival rates were poorer ( $46 \%$ at 5 years) for patients diagnosed with both high T (T3 or T4) and M (M1 through M3) stage disease (Gajjar et al., 2006). Patients who received post-operative adjuvant radiotherapy and chemotherapy had a significant survival advantage over those who received radiotherapy alone (Robertson, 2006). Over the last decade, the continuously improved therapy resulted in the five year progression free survival rate of approximately $80 \%$ for patients with average-risk disease and 60\% for patients with high-risk disease (Merchant et al., 2007; Gajjar et al., 2006; Packer et al., 2006; Gottardo and Gajjar, 2006).

Adjuvant chemotherapy has also been a common modality for glioma treatment. Chemotherapy with a combination of multiple agents (e.g. procarbazine, lomustine, vincristine) have improved the quality of life in adult patients with malignant astrocytomas (Taphoorn et al., 2007; Rabbani et al., 2007). However the clinical benefits were not consistently observed in pediatric patients. In an early high grade astrocytoma trial from Children's Cancer Study Group, five-year event-free survival was seen in 46\% of patients in the radiotherapy and chemotherapy group, and only $18 \%$ of patients in the radiotherapy-alone group (Sposto et al., 1989). In a recent Pediatric Oncology Group (POG) study, 40 patients with high grade gliomas were treated with radiotherapy and adjuvant chemotherapy (procarbazine or topotecan). The 3-year OS and PFS were only $15 \%$ and $10 \%$, respectively (Chintagumpala et al., 2006). In a Children's Oncology Group (COG) study, 113 patients with CNS tumors were treated with temozolomide the dosage of which was based on prior radiation treatment status. Among 75 glioma patients, only 1 partial response was observed. This is in contrast to 1 complete response and 3 partial responses out of 29 medulloblastoma patients (Nicholson et al., 2007). In a recent trial in children with high grade astrocytoma, with a total of sixteen patients enrolled, five year PFS was $28.6 \%$ (Sanders et al., 2007). These studies suggest effective chemotherapy for pediatric glioma patients is still lacking.

In summary, the role of conventional chemotherapy in pediatric brain tumor treatment is very uncertain. Although a portion of medulloblastoma patients have benefited from conventional chemotherapy to some degree, such similar benefits have not been observed in high grade astrocytoma patients. Treatment failure is multi-factorial and can be attributed to issues such as intrinsic tumor resistance or to therapy related issues such as optimal dosing strategy or optimal drug delivery failure. Although each of these aspects warrants further investigation, novel agents are still urgently needed. 


\subsubsection{Camptothecin analogs}

The camptothecin analogs are a promising group of novel agents that have shown wide antitumor activity. The anti-tumor activity of the parent 20(S)-camptothecin, a natural alkaloid from the Chinese tree Camptotheca acuminata, was first described in the 1960s (Wall and Wani, 1977; Kepler et al., 1969) The natural compound, however, demonstrated unpredictable toxicities in clinical trials including hemorrhagic cystitis (Hertzberg et al., 1989; Wall and Wani, 1977). The conversion of the open carboxylate form into the closed lactone form at the acidic $\mathrm{pH}$ of bladder was considered to be responsible for the severe hemorrhagic cystitis, which could be life-threatening (Lansiaux et al., 2007). Different strategies were developed to modify the compound to stabilize the closed lactone form and reduce the adverse reactions. Currently only two camptothecin analogs, topotecan and irinotecan, have been approved by Food and Drug Administration (FDA) for clinical use. Topotecan is indicated for small cell lung cancer and metastatic ovarian cancer after failure of first-line chemotherapy (Kudelka et al., 1996; Lynch, Jr. et al., 1994). Irinotecan is indicated for metastatic colorectal cancer usually with other drugs (Rothenberg et al., 1996; Sasaki et al., 1994; Shimada et al., 1993). As a prodrug, irinotecan is converted by carboxylesterase to its active metabolite SN-38 in vivo.

The ester bond of the lactone E ring of camptothecin analogs (Figure 1-1) undergoes a reversible $\mathrm{pH}$-dependent hydrolysis, with a closed-lactone ring form at acidic conditions and open-carboxylate ring form at basic conditions. Under physiological conditions ( $\mathrm{pH}$ 7.2-7.4) the carboxylate form predominates. However only the lactone form of these compounds is responsible for their biological activity to interact with the topoisomerase-I and DNA complex (Hertzberg et al., 1989; Capranico et al., 2007; Marchand et al., 2006). The structures of topotecan and irinotecan and $\mathrm{pH}$-dependent conversions are shown in Figure 1-1.

\subsubsection{Preclinical studies of topotecan and irinotecan for brain tumor treatment}

In rodent models of pediatric brain tumors, both topotecan and irinotecan showed potent anti-brain tumor activities (Friedman et al., 1994; Hare et al., 1997; Pawlik et al., 1998; Zamboni et al., 1998). The activity of topotecan was evaluated against a panel of xenografts derived from ependymomas (D528 EP, D612 EP), childhood high-grade gliomas (D-456 MG, D-212 MG), adult high-grade gliomas (D-245 MG, D-54 MG), and medulloblastomas (D425 Med) growing subcutaneously (s.c.) in athymic nude mice. Topotecan was given at a dose of $1.9 \mathrm{mg} / \mathrm{kg}$ by intraperitoneal (i.p.) on days 1-5 and 812 , which represents the dose lethal to $10 \%$ of treated animals. Topotecan was active in the therapy of all s.c. xenografts tested, with growth delays ranging from 6.3 days in D$54 \mathrm{MG}$ to 55.7 days in D528 EP. Topotecan produced statistically significant tumor regressions in D425 Med, D-456 MG, D-245 MG, D528 EP, and D612 EP. No tumor regressions were seen in any control animal (Friedman et al., 1994). This study demonstrated topotecan was effective against CNS tumors implanted as s.c. xenografts in mice. 

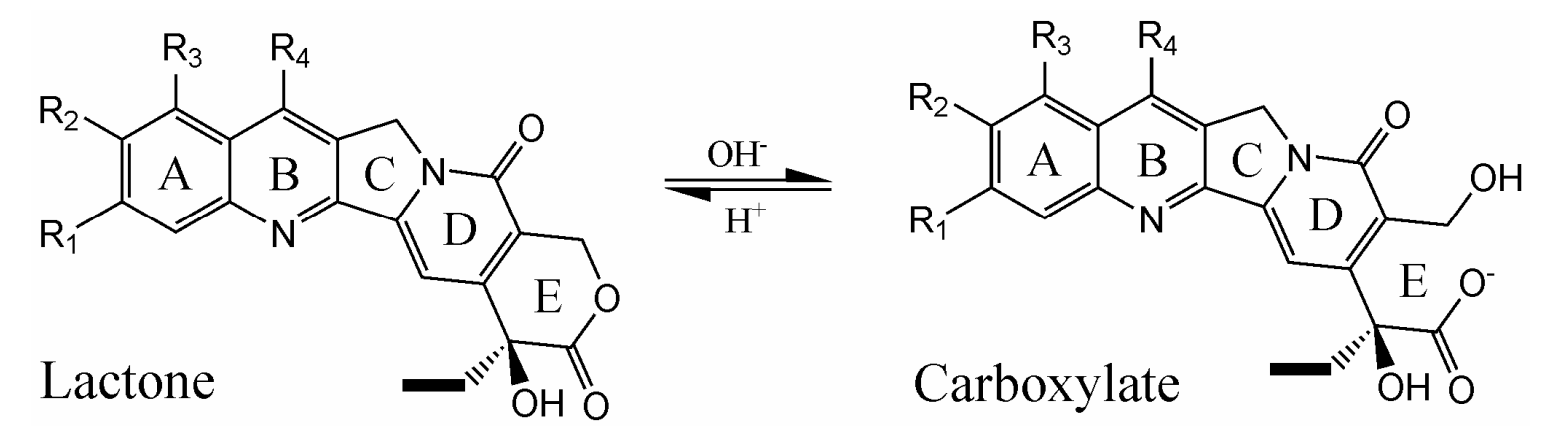

\begin{tabular}{|c|c|c|c|c|}
\hline & $\mathrm{R}_{1}$ & $\mathrm{R}_{2}$ & $\mathrm{R}_{3}$ & $\mathrm{R}_{4}$ \\
\hline Topotecan & $\mathrm{H}$ & $\mathrm{OH}$ & $-\mathrm{CH}_{2} \mathrm{~N}_{\left(-\mathrm{CH}_{3}\right)_{2}}$ & $\mathrm{H}$ \\
\hline Irinotecan & $\mathrm{H}$ & $\mathrm{OH}$ & $\mathrm{H}$ & $-\mathrm{CH}_{2} \mathrm{CH}_{3}$ \\
\hline SN-38 & $\mathrm{H}$ & $\mathrm{OH}-\mathrm{CH}_{2}$ & $\mathrm{H}$ \\
\hline
\end{tabular}

Figure 1-1 Structures of camptothecin analogs.

Chemical structures of camptothecin analogs and $\mathrm{pH}$ dependent conversion between lactone and carboxylate forms. At acidic conditions the lactone form dominates; at basic conditions the carboxylate dominates. 
Houghton and colleagues studied the efficacy of topotecan and irinotecan against a panel of 21 human tumor xenografts from adult and pediatric malignancies. Many of the xenografts represented sublines resistant to conventional chemotherapy. All tumors were grown subcutaneously. Topotecan was administered by oral gavage 5 days per week for 12 consecutive weeks at dosages up to $1.5 \mathrm{mg} / \mathrm{kg}$. Irinotecan was given by i.v. administration daily for 5 days each week for 2 weeks [( $\mathrm{d} \times 5) 2]$ (one cycle of therapy), repeated every 21 days at the dosage up to $10 \mathrm{mg} / \mathrm{kg}$ maximum tolerated dose. A pediatric glioblastoma line (SJG-2) had a 100\% complete response (CR) to irinotecan and $80 \% \mathrm{CR}$ to topotecan. A pediatric medulloblastoma line had a $100 \% \mathrm{CR}$ to topotecan and $88 \% \mathrm{CR}$ to irinotecan (Houghton et al., 1995). This study demonstrated both topotecan and irinotecan had outstanding anti-tumor activities in various human tumor xenografts including CNS tumors.

The promising anti-tumor activity of camptothecin analogs were further confirmed in other CNS tumor models. Hare and colleagues reported irinotecan induced complete response in two brain tumor (D456 high grade glioma, D341 medulloblastoma) s.c. xenografts in athymic mouse models when irinotecan was given at a dosage of 40 $\mathrm{mg} / \mathrm{kg}$ via intraperitoneal injection on days 1-5 and 8-12 (Hare et al., 1997). Compared with more than 40 conventional drugs tested including alkylators, antimetabolites, vinca alkaloids, anthracyclines, and L-asparaginase (Friedman et al., 1983; Friedman et al., 1986), irinotecan has demonstrated the most potent anti-CNS tumor activity. Vassal et al. evaluated irinotecan in 5 advanced stage subcutaneous medulloblastoma xenografts in nude mice. With a 5-day dosing schedule, the highest i.v. dose (40 mg/kg/day) induced complete regressions in four of five xenografts. To study the dosing schedule dependency of its anti-tumor activity, irinotecan was given i.v. at the same total doses over the same period (33 days) using either a protracted or a sequential schedule. With the protracted schedule, 3 out of 6 animals were tumor free on day 378. The sequential schedule failed to induce any CR (Vassal et al., 1997). This study suggested the efficacy of irinotecan might depend on dosing schedule. Nakatsu et al. tested SN-38, the irinotecan active metabolite, in a few CNS tumor cell lines. They demonstrated that SN-38 had strong antitumor effects on GB-1 and U-87MG two human glioblastoma cell lines with multidrug resistance phenotype (Nakatsu et al., 1997).

In summary, the camptothecin analogs demonstrated potent anti-tumor activities in various CNS tumor cell lines and xenograft animal models. Due to the success of these preclinical studies, this class of compounds has been advanced to clinical trials for CNS tumors.

\subsubsection{Clinical studies with Camptothecin analogs for brain tumor treatment}

A Canadian group has conducted a phase II trial of topotecan in patients with malignant glioma. Topotecan $\left(1.5 \mathrm{mg} / \mathrm{m}^{2}\right.$ i.v. over 30 minutes $)$ was given daily by 5 days every 3 weeks [( $\mathrm{d}$ 5) x 3]. Among 31 patients enrolled, one had complete radiographic response, and one had partial response (PR). With this dose and schedule, topotecan showed modest activity in recurrent glioblastoma and anaplastic astrocytoma (Macdonald et al., 1996). The results were further corroborated in another phase II study in adults 
with primary malignant glioma. With 63 patients enrolled and topotecan given at a dose of $2.6 \mathrm{mg} / \mathrm{m}^{2}$ over a 72-hour infusion weekly, only 4 PRs were observed from newly diagnosed glioblastoma (GBM) or anaplastic astrocytoma (AA). No response was observed from recurrent GBM (Friedman et al., 1999a). These results suggest that topotecan has modest activity against malignant gliomas and further evaluation of its effectiveness is necessary when alternative schedules or combination regimens are available.

Blaney and colleagues initiated a phase II study in pediatric patients with CNS tumors, including high grade gliomas, medulloblastomas, and brain stem tumors. Topotecan was administered as a 24-hour infusion starting from $5.5 \mathrm{mg} / \mathrm{m}^{2}$ with escalation to $7.5 \mathrm{mg} / \mathrm{m}^{2}$ in patients who did not experience dose-limiting toxicity. Disappointingly no CR or PR was observed. Topotecan was inactive in this group of patients with this dosing schedule (Blaney et al., 1996).

The German Society for Pediatric Oncology and Hematology conducted a phase II study in which topotecan was administered orally in ice-cold orange juice with a starting dosage of $0.4 \mathrm{mg} / \mathrm{m}^{2} /$ day and escalating to maximum tolerated dose. Among 32 patients, $1 \mathrm{CR}$ and $2 \mathrm{PR}$ were observed. This study demonstrated that topotecan was only mildly effective against recurrent pediatric high-grade glioma (Wagner et al., 2004).

Stewart and colleagues reported in a phase II trial of high-risk medulloblastoma and supratentorial primitive neuroectodermal tumor, in which topotecan dosing was pharmacokinetically guided to reach an exposure duration threshold (EDT) (Stewart et al., 2004a). Pharmacokinetic studies were conducted for each patient on day 1 to reach topotecan lactone area under the plasma concentration-time curve (AUC) of 120$160 \mathrm{ng} / \mathrm{ml} \cdot \mathrm{h}$ (Stewart et al., 2004a; Zamboni et al., 1998). Topotecan was initially administered as 30-minute infusion. After 10 patients were enrolled, the infusion was modified to a four-hour infusion (Zamboni et al., 1998). Target plasma topotecan AUC was achieved in 24 patients. Among 36 assessable patients, 4 had a CR and 6 had a PR (Stewart et al., 2004a). This study demonstrated topotecan was an effective agent against pediatric medulloblastoma when topotecan was administered on a pharmacokinetically guided dosing schedule.

However, the promising results were not seen in a recent phase II pediatric highgrade glioma study. Topotecan was given as 30-minute infusion and plasma pharmacokinetic studies were conducted to estimate topotecan exposure level. Of 14 patients enrolled, no CR or PR was observed. 3-year OS is 14\% and 3-year PFS is only 7\% (Chintagumpala et al., 2006).

Irinotecan has also been extensively tested in clinical trials for CNS tumors. In phase I trials, several different dosing schedules and maximum tolerated dose (MTD) have been investigated. Since many brain tumor patients receive enzyme-inducing anticonvulsants (EIAs), which may significantly affect irinotecan clearance, the MTD of irinotecan was determined for patients with or without EIA treatment. In an adult recurrent malignant glioma trial, irinotecan was given over a 90-minute i.v. infusion 
weekly for 4 weeks every 6 weeks. The MTD for EIA treatment group was determined to be $411 \mathrm{mg} / \mathrm{m}^{2} /$ week and $117 \mathrm{mg} / \mathrm{m}^{2} /$ week for patients without EIA (Gilbert et al., 2003). Prados and colleagues evaluated another dosing schedule in which irinotecan was given every 3 weeks (90-minute i.v. infusion). For EIA treatment group the MTD of irinotecan was determined to be $750 \mathrm{mg} / \mathrm{m}^{2} /$ dose and $350 \mathrm{mg} / \mathrm{m}^{2} /$ dose for non-EIA treatment group (Prados et al., 2004).

However with promising results from preclinical studies and well-defined phase I studies, the clinical benefit from phase II studies with irinotecan for brain tumors were below expectations. In a phase II adult recurrent glioma trial where a weekly dosing schedule was used, Friedman and colleagues reported that of 60 patients enrolled, 9 PR were confirmed and no CR was observed (Friedman et al., 1999b). In this study, the patients were not dosed based on the EIA treatment status. However when irinotecan was dosed on EIA treatment status in another phase II adult malignant glioma trial, of 18 patients enrolled only 1 CR was observed (Batchelor et al., 2004). Turner and colleagues conducted a study in children with high risk malignant brain tumors. Irinotecan was given as a 90-minute i.v. infusion at a dosage of $125 \mathrm{mg} / \mathrm{m}^{2}$ weekly for 4 weeks every 6 weeks. 2 CRs and 2 PRs were observed in total 22 patients (Turner et al., 2002). The every-3week dosing schedule has also been tested in a few phase II trials. In an adult recurrent malignant glioma trial, Cloughesy and colleagues reported 2 PRs out of a total 14 patients (Cloughesy et al., 2002). In another multi-center phase II study for adult glioblastoma, only 1 PR and 7 minor responses were observed out of a total 46 patients (Raymond et al., 2003). In an adult glioblastoma multiforme trial, all patients demonstrated progressive diseases (Chamberlain, 2002). Most recently Blaney and colleagues conducted a phase II study in pediatric solid tumor patients, which included children with recurrent primary CNS tumors. Among the patients with CNS tumors, responses were only observed in those with medulloblastomas (Bomgaars et al., 2007).

In summary, although camptothecin analogs (topotecan and irinotecan) have demonstrated modest efficacy against brain tumors, the clinical outcomes did not reproduce the encouraging results seen in the preclinical studies. Several conclusions can be made from the results of these clinical trials. Topotecan and irinotecan were more effective in medulloblastoma than in gliomas (Stewart et al., 2004a; Bomgaars et al., 2007). Clinical response of irinotecan was far below expectations, even lower than in some existing chemotherapies (Raymond et al., 2003). Tumors fail to respond to chemotherapy for many reasons, one of which appears to be drug resistance. However the fact that most of the preclinical models were constructed on s.c. implanted xenografts, which is not orthotopic for brain tumors, suggests that drug delivery to the brain of the patients may not be adequate. This inadequacy may be due to the presence of blood brain barrier (BBB) and blood cerebrospinal fluid (CSF) barrier (BCB).

\subsection{Blood brain barrier (BBB) and blood CSF barrier (BCB)}

The BBB is a collective term used to describe the phenotype that regulates the material penetration into or out of the brain and protects the brain from endogenous or 
xenobiotic toxins. The existence of the BBB was first described by a German bacteriologist Paul Ehrilich (1902) who observed that systemically injected dye stained all the organs of an animal except brain. The concept of the BBB was formally proposed by a Russian biochemist Lina Stern in 1921. The actual membrane structure of the BBB wasn't demonstrated until the advent of scanning electron microscope in 1950s (Dempsey and Wislocki, 1955; Van, V and Clemente, 1955). Most of our understanding of the molecular structures and functions of the BBB has been achieved during the last three decades. The presence of the BCB was first described by Edwin Goldmann (Ehrilich's student) in 1913 when he injected a dye into the CSF of the brain directly. Only the brain was stained but not the rest of the body. Since the CSF forms a distinct compartment from the extracellular fluid (ECF) of brain parenchymal tissue, the concept of the $\mathrm{BCB}$ should be distinguished from the BBB.

The main focus of this work is to elucidate the mechanisms that control topotecan penetration into the brain. This discussion will primarily focus on the properties of the $\mathrm{BBB}$ or the $\mathrm{BCB}$ related to drug penetration.

\subsubsection{Blood brain barrier}

Structurally the BBB consists of multiple components. As depicted in Figure 1-2, at least three types of cells are involved to maintain the integrity of the BBB. The single layer of cerebral vascular endothelial cells, which are joined together by tight junctions, forms the pivotal component of the BBB (Davson and Segal, 1996). The pericytes and astrocytic foot process, which are closely associated with the endothelial cells, further strengthen the BBB (Abbott, 2005; Pardridge et al., 1986). Compared with other organs, the endothelial cells at the BBB have a particularly higher content of mitochondria suggesting active energy dependent activities (Oldendorf et al., 1977). In addition to providing physical support, the pericytes and astrocytes in the BBB also play regulatory roles (Zozulya et al., 2007; Abbott, 2002; Balabanov and Dore-Duffy, 1998).

Functionally, the BBB is to maintain the homeostatic brain microenvironment by selectively transporting nutritional materials into the brain and excluding toxic metabolites or xenobiotics out of the brain. To achieve the task, first the unique structure of the BBB greatly restricts paracellular diffusion. Second, compared with the vascular endothelia in other tissues, low pinocytotic and transcytotic activity of brain endothelial cells results in very limited non-specific trans-endothelial transport (Vorbrodt and Dobrogowska, 2003; Davson and Segal, 1996). However due to the nature of the plasma membrane, highly lipophilic small molecules are still allowed to diffuse through the endothelial cells. Third, the transport of solutes and nutrients including inorganic ions, sugars, amino acids, nucleic acids, and small peptides is selectively mediated by specialized proteins such as ATP-binding cassette (ABC) transporters and solute carriers (Vorbrodt and Dobrogowska, 2003; Ohtsuki and Terasaki, 2007).

The carrier-mediated transport system refers to all the solute carrier (SLC) members that are classified into 47 families (Hediger et al., 2004). Among them, the 


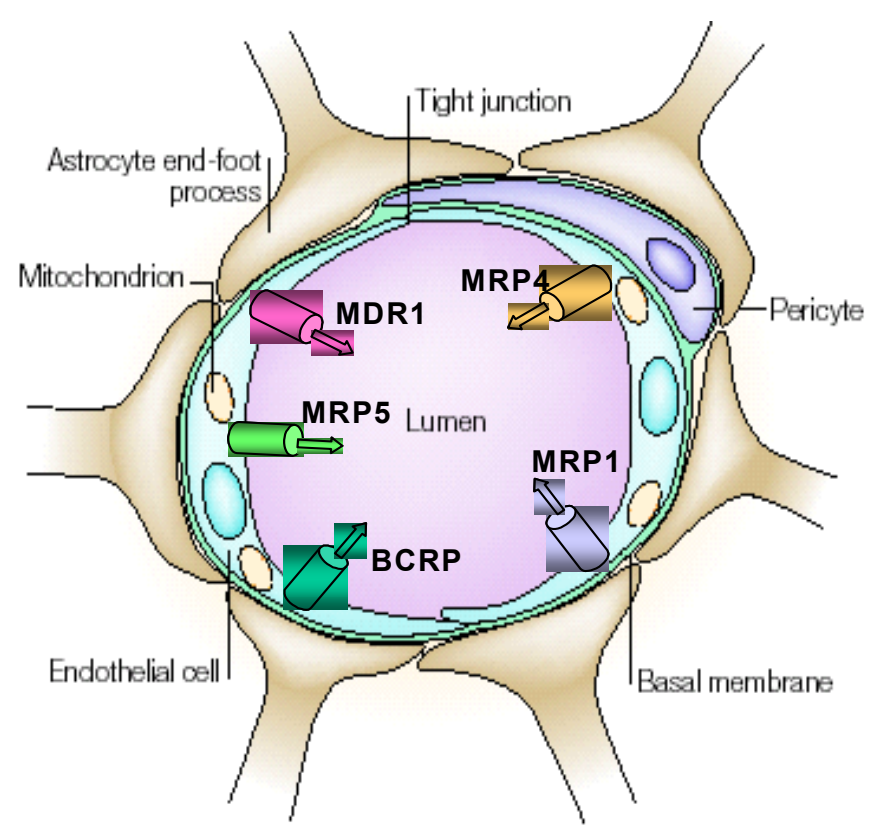

\section{Figure 1-2 Blood brain barrier.}

The specialized brain capillary endothelial cells sealed by tight junctions form the blood brain barrier (BBB). Brain capillary and pericytes are surrounded by a basal membrane, which is tightly ensheathed by the astrocytic end-feet. ABC transporters such as MDR1(P-gp), MRP1, MRP4, MRP5, and BCRP are localized at the apical side of the endothelial cells.

Source: Modified with permission. Loscher W, Potschka H, 2005. Drug resistance in brain diseases and the role of drug efflux transporters. Nat. Rev. Neurosci. 6: 591-602. 
family $S L C 21$, the organic anion transporting polypeptides $(O A T P)$ and family $S L C 22$ organic anion/cation transporters $(O A T / O C T)$ are the most important in terms of drug transport (Ohtsuki and Terasaki, 2007; Loscher and Potschka, 2005a). These transporters are not directly coupled with ATP but rather driven by electrochemical potential difference created by various ion gradients. Depending on the sub-cellular localization (apical or basolateral) on the endothelial cells these carrier-transporters can move substrates either into or out of the brain (Ohtsuki and Terasaki, 2007; Ito et al., 2005c).

Another mechanism of transport at the BBB is mediated through the family of ATP-binding cassette (ABC) transporters. P-glycoprotein (P-gp/MDR1), the prototype of ABC transporters, is widely expressed through the body including BBB (Schinkel et al., 1994; Thiebaut et al., 1987). Although P-gp was identified three decades ago (Juliano and Ling, 1976) its functional significance at the BBB wasn't clearly demonstrated until the generation of the $M d r l a^{(--)}$mouse model (Schinkel et al., 1994). Schinkel and colleagues clearly have shown the Mdrla deficient mouse was more sensitive to the neurotoxic pesticide ivermectin (100-fold) and anti-cancer drug vinblastine (3-fold) than wild type mice (Schinkel et al., 1994). After this study, the expression of ABC transporters in the brain was profiled in humans and rodents. Until now the $\mathrm{ABC}$ transporters that have been detected on protein level at the BBB include P-gp (MDR1), MRP1 ( $A B C B 1)$, MRP4 $(A B C B 4)$, MRP5 ( $A B C B 5)$, and BCRP ( $A B C G 2)$ (Ito et al., 2005c; Loscher and Potschka, 2005b; Potschka et al., 2003; Zhang et al., 2000; Nies et al., 2004; Cordon-Cardo et al., 1990; Rao et al., 1999) in both rodents and human. Particularly high level of both mRNA and protein of P-gp and BCRP were detected at BBB in human and rodents, which suggests they probably play a bigger role as a barrier(Cooray et al., 2002; Hori et al., 2004; Schinkel et al., 1994). On the contrary, only low levels of mRNA were detected for transporters such as MRP2 and MRP3, which suggests they probably do not play a significant role at BBB (Zhang et al., 2000).

A recent study indicated that the $\mathrm{ABC}$ transporters were associated with different cell types at the BBB (Yousif et al., 2007) although the functional relevance of such expression pattern is not clear. While MRP1 and MRP5 were quite ubiquitously expressed through the BBB, MDR1, MRP4, and BCRP were closely associated with endothelial cells. MRP2 and MRP3 were closely associated with pericytes (Yousif et al., 2007). Another feature about these transporters is they are strict efflux transporters, which means they only move substrates out of the cells where they are expressed. Current evidence has shown all the expressed ABC transporters are located at the apical side of $\mathrm{BBB}$, which suggests they only efflux substrates from brain side into blood (Ito et al., 2005c).

\subsubsection{Blood cerebrospinal fluid barrier}

The blood cerebrospinal fluid (CSF) barrier (BCB) consists of the choroid plexus (CP) and arachnoid membrane. Only the $\mathrm{CP}$ has been extensively studied for drug transport and metabolism (Johanson et al., 2005). In this work whenever BCB is mentioned, it is an expression of the choroid plexus related structure. As depicted in 
Figure 1-3, the $\mathrm{CP}$ is composed of an outer layer of ependymal epithelial cells and an inner layer of capillary endothelial cells. The distinct feature of the $\mathrm{BCB}$ is the tight junction is absent between the endothelial cells but present between the ependymal epithelial cells. This feature results in a fenestrated capillary but a much less permeable layer of epithelial cells (Redzic and Segal, 2004). Although the choroid plexus is only distributed in the cerebral ventricles, the total surface area is the same order of magnitude as the total BBB due to the lush apical membrane microvilli (Keep and Jones, 1990).

Functionally, CP serves as a secretory and metabolizing organ, which is often described as a mini kidney and liver of the brain (Strazielle and Ghersi-Egea, 2000). The total volume of CSF in human is estimated 150-270 ml, which is renewed about 5 times a day (Kohn et al., 1991; Davson and Segal, 1996). Furthermore the choroidal vascular perfusion is five to ten times that of the cerebral blood flow (Faraci et al., 1994). 85\% of the CNS sodium chloride is secreted through choroid plexus compared with $15 \%$ through the BBB (Smith et al., 1981). These facts suggest that the choroid plexus is an important secretory organ. Similar to the endothelial cells of the BBB, the choroid plexus has a high capacity for drug metabolism and ATP production for energy dependent activities (Strazielle and Ghersi-Egea, 2000).

In addition to secreting $\mathrm{CSF}$, the $\mathrm{CP}$ is also a barrier. The tight junctions joining the epithelial cells greatly diminish the paracellular diffusion across choroid plexus (Zheng et al., 2003). Likewise various types of carriers and transporters regulate the penetration of solutes, nutrients and xenobiotics. Multiple carriers from SLC21 (OATP) and $S L C 22$ (OAT/OCT) families are expressed in the CP. For examples, in rodents Oat3 is located at the basolateral side of the CP epithelial cell of the BCB (Nagata et al., 2002), while Oatp3 and Oct2 are located at the apical side (Gao et al., 1999). In rodents, Oatp2 has been found at both sides (Ito et al., 2005c). ABC transporters are also expressed at the $\mathrm{BCB}$. In both human and rodents MRP1 and MRP4 are expressed at the basolateral side of the BCB (Nies et al., 2004; Leggas et al., 2004a) and P-gp and Bcrp are expressed at the apical side (Zhuang et al., 2006). Depending on the subcellular localization (either apical or basolateral), these $\mathrm{ABC}$ transporters might have a different impact on CSF penetration of their substrates.

\subsection{The effect of $\mathrm{ABC}$ transporters on camptothecin analogs $\mathrm{CNS}$ penetration}

Many factors affect CNS drug penetration, including the drug physiochemical properties, cerebral blood flow, enzymatic metabolism barrier (Johnson and Anderson, 1996; Ghersi-Egea et al., 1988), and expression of influx or efflux transporters (Loscher and Potschka, 2005b). Camptothecin analogs are highly lipophilic drugs with a calculated octanol/water partition coefficient (cLogP) of 407 for topotecan lactone and 585 for irinotecan lactone (ChemDraw 9.0.1, CambridgeSoft Corporation, Cambridge, MA). The brain is a well perfused organ and topotecan is not a heavily metabolized drug. Thus, the topotecan CNS penetration is most likely controlled by active processes, either influx or efflux transporter system (e.g. ABC transporters). 


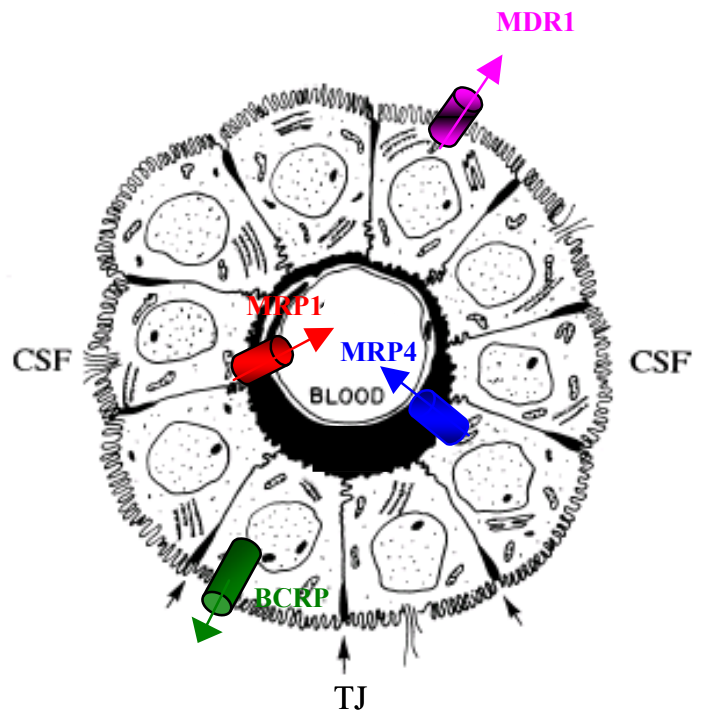

\section{Figure 1-3 Blood cerebrospinal fluid barrier.}

Cross-section of the blood-CSF barrier (BCB) at the choroid plexus. Choroidal epithelia with tight junctions (TJ) forms a dynamic interface between two circulating fluids, the choroidal blood and ventricular CSF. The capillaries of the plexus are fenestrated. Abundant microvilli at the apical side of the choroidal epithelia and the basolateral infoldings greatly expand the surface area for molecular exchange at the $\mathrm{BCB}$. ABC transporters such as MRP1 and MRP4 are localized at the basolateral side of the choroidal epithelia, whereas MDR1 (P-gp) is present at the apical side of choroidal epithelia.

Source: Modified with permission. Johanson CE, Duncan JA, Stopa EG, Baird A, 2005. Enhanced prospects for drug delivery and brain targeting by the choroid plexus-CSF route. Pharm. Res. 22: 1011-1037. 


\subsubsection{The role of $\mathrm{ABC}$ transporters in vitro studies}

Numerous in vitro studies have demonstrated camptothecin analogs are substrates for several ABC transporters. P-gp has been shown to confer resistance to various anticancer drugs including camptothecin analogs (Gottesman et al., 2002). Chen and colleagues first presented evidence that topotecan mediated cytotoxicity was reduced in a P-gp over-expressing cell line KB V1, and P-gp inhibitor verapamil restored the topotecan sensitivity (Chen et al., 1991). Hendricks et al. measured the topotecan IC50 values in a colchicine-resistant cell line CHR5 (a typical P-gp overexpressing line). Examination of the IC50 values observed in colony-forming assays revealed that the CHRC5 cells were 15-fold more resistant to topotecan after a 1-h exposure compared with parental line (Hendricks et al., 1992b). Chu and colleagues demonstrated that the Pgp overexpessing cell line KB-C2 was 6.3-fold more resistant to irinotecan than the parental cells (Chu et al., 1999b).

BCRP was also well documented to be heavily involved in transport of several camptothecin analogs. Yang et al. first reported mitoxantrone-resistant human breast carcinoma cell line, MCF7/MX was cross-resistant to camptothecin analogues including topotecan (180-fold), 9-aminocamptothecin (120-fold), irinotecan (56-fold), and SN-38 (101-fold) (Yang et al., 1995). Later it was demonstrated in mouse fibroblast cell lines that lack functional $M D R 1$ and $M R P 1$ genes, resistance to topotecan was mediated through BCRP gene amplification and overexpression (Brangi et al., 1999; Allen et al., 1999). Maliepaard and colleagues reported topotecan directly induced BCRP overexpression in an ovarian tumor cell line (Maliepaard et al., 1999a). Efforts were made to circumvent the BCRP mediated resistance by using non-specific inhibitor GF120918 (Maliepaard et al., 2001c). In addition to the tumor cells, BCRP was also reported to be expressed in various normal tissues of multiple species, which suggests BCRP plays an important role in camptothecin disposition (Maliepaard et al., 2001a; Cooray et al., 2002; Eisenblatter et al., 2003; Cisternino et al., 2004).

MRP4 is another ABC transporter reported to confer resistance to camptothecin analogs. Norris et al. reported intracellular SN-38 concentrations were reduced at least 4fold in MRP4 overexpressing HEK/293 cells. Functionally the MRP4 transfectants are 5times more resistant to irinotecan (Norris et al., 2005). Tian and colleagues performed a series of studies to compare the resistance to various camptothecin analogs conferred by MRP4. Based on the results from the MTT assay, with $48 \mathrm{~h}$ exposure time of the test drug, MRP4 conferred resistance to CPTs tested in the order 10-OH-CPT (14.21) $>$ SN38 carboxylate $(9.70)>$ rubitecan $(9.06)>\mathrm{SN}-38$ lactone $(8.91)>\mathrm{CPT}$ lactone $(7.33)>$ CPT-11 lactone (5.64) > CPT carboxylate (4.30) > CPT-11 carboxylate (2.68) (Tian et al., 2005). They further demonstrated in a separate study that MRP4 conferred a 12-fold resistance to topotecan in the 4-hour drug-exposure MTT assay (Tian et al., 2006). Another MRP family member, MRP2, was also shown to have low affinity to irinotecan at high concentrations (e.g., $250 \mu \mathrm{M})$ (Chu et al., 1999a) .

In summary, results of in vitro studies have identified that at least three $\mathrm{ABC}$ transporters including P-gp, BCRP, and MRP4 confer resistance to camptothecin analogs. 
Based on the results of cytotoxicity assays, BCRP is an efficient transporter for topotecan whereas P-gp and MRP4 also transport topotecan but with much less efficiency. However, these in vitro results need to be confirmed in vivo before any therapeutic effort on modulating these transporters can be done.

\subsubsection{The utilization of targeted mutation mouse models of $\mathrm{ABC}$ transporters}

As in vitro results (e.g. drug accumulation assay or cytotoxicity assay) are not always translated in vivo, it is important to verify the exact role of these ABC transporters in drug disposition in vivo. The targeted mutation (knockout) mouse models provide unique tools to address these issues. Several knockout mouse models for various ABC transporters have been generated by different labs including $M d r l a^{(--)}, M d r l a / b^{(--)}$, $B c r p 1^{(--)}$. With further breeding of these mice, multiple transporters knockout mouse models can be generated such as Bcrpl/Mdrla/ $b^{(--)}$(Jonker et al., 2005). The role of P-gp in vivo for vinblastine CNS penetration has been clearly defined by using $M d r 1 a^{(-/)}$ model (Schinkel et al., 1994). de lange et al. reported a typical P-gp substrate, rhodamine123, brain concentration was 4-fold higher in $M d r l a^{(-/)}$mice than wild type (de Lange et al., 1998). The brain-to-plasma concentration ratio of an investigational drug CP-615003 in $M d r l a / b^{(--)}$was 7-times that in wild type (Venkatakrishnan et al., 2007). These studies strongly suggest P-gp plays critical role in drug CNS disposition.

Other in vivo studies utilizing knockout models do not seem to support in vitro results. For example, although in vitro irinotecan and SN-38 were substrates for MRP4, which also functionally conferred resistance in cell lines, they showed unchanged pharmacokinetics in $\operatorname{Mrp}^{(--)}$mice (unpublished data). Most recently de Vries and colleagues reported the topotecan concentrations from whole brain homogenate in Mrp $4^{(-}$

${ }^{-)}$were not substantially different from those in wild type mice(de Vries et al., 2007). These results could be explained by the overlapping substrate specificity of the transporters or, in other words, functional redundancy. It strongly indicates that if a drug is a substrate for a transporter in vitro, it does not necessarily translate to a significant role in vivo. The fact that a drug is usually a substrate for multiple transporters, makes it more difficult to distinguish the role of a single transporter on drug disposition and penetration into local tissues (e.g., central nervous system). These results warrant further investigation of the role of the $\mathrm{ABC}$ transporters in the CNS penetration of camptothecin analogs. The in vivo role of $\mathrm{ABC}$ transporters in camptothecin CNS penetration can be determined by comparing CNS drug penetration in the transporter deficient models with that in wild type mice. 


\subsection{Methods to evaluate CNS drug penetration}

\subsubsection{Homogenization and autoradiography method}

Several methods can be used to determine the drug concentration and distribution in brain. The most common method is to directly collect the brain and extract total drug from the brain homogenate. This is the method that was used in the early drug distribution studies (Slordal et al., 1988; Nierenberg et al., 1991; Jain, 1989) and is still routinely used (Straathof et al., 1999; de Vries et al., 2007; Dickson et al., 2007). However, the homogenization method does not provide information that is crucial to understand the mechanism of CNS drug penetration. This approach mixes drug from three pharmacologically distinct compartments of the brain including capillary vessels, extracellular fluid (ECF), and intracellular fluid of brain parenchymal tissue. With this method, the drug that is not bound to protein cannot be separately determined from the protein bound drug. This determination is critical as only the unbound faction of a drug is pharmacologically active and available for transport. Autoradiography is able to offer information on drug spatial distribution, but it does not discriminate parent drugs from the metabolites and just like homogenization it does not separate the unbound drug from the protein bound (Shapiro et al., 1988). To understand the mechanism of drug transport in the brain, it is crucial to sample unbound drug in specific compartments of the brain (e.g., ECF, vCSF). Currently, microdialysis is the only technique that is capable of this.

\subsubsection{Microdialysis}

Microdialysis was originally developed to monitor neurotransmitters in the living brain (Bito et al., 1966; Ungerstedt and Pycock, 1974). Very quickly this sampling technique found applications in drug delivery studies (Elmquist and Sawchuk, 2000). For the determination of CNS drug penetration, this technique has many advantages over the common method such as whole tissue homogenization. Advantages include continuous sampling without fluid loss, measurement of discrete anatomic compartments, clean samples suited for direct bioanalysis without prior processing, and measurement of unbound or pharmacologically active moieties (Benveniste and Huttemeier, 1990). However, microdialysis sampling does have disadvantages, including the fact that it is an invasive technique. The potential damage from probe implantation has been an issue debated in the microdialysis literature for many years. In the 1980s, a series of studies were performed to address this concern. Particularly, Benveniste and colleagues designed a study to evaluate the effect of probe implantation on tissue damage at the blood brain barrier (Benveniste et al., 1987). They demonstrated in rats that the local cerebral blood flow and glucose uptake returned to normal levels as early as 24 hours after implantation of a microdialysis probe. These results suggested the tissue damage could be managed if animals were given enough time to recover. Other disadvantages include that probes have to be calibrated in vivo and more sensitive analysis methods are usually required due to the small sample volume. Although microdialysis sampling does have these potential 
concerns, the ability to directly address research questions related to CNS drug penetration makes this methodology particularly appealing.

\subsubsection{Probe material and perfusion fluid}

The functional part of a probe, the membrane, can be made of different materials including cellulose, copolymers, and polysulfone (Levine and Powell, 1989). These materials offer different molecular weight cut-off and permeability to solutes (Hernandez et al., 1986). It is also important that the membrane does not interact with the drug as this may affect dialysis result. The probes could be home-made or purchased commercially. Two major vendors provide microdialysis probes, and they are available in many different sizes (CMA microdialysis AB, Stockholm, Sweden; Bioanalytical systems, West Lafayette, IN, USA).

In addition to the probe material, the perfusion fluid is also critical to the microdialysis results. In principle, the composition and $\mathrm{pH}$ of the perfusion fluid should be as close as to the fluid of the tissue in study. For example, artificial cerebrospinal fluid (aCSF) is usually used for microdialysis in CNS as it mimics the composition and $\mathrm{pH}$ of CSF ( $\mathrm{NaCl} 148 \mathrm{mM}, \mathrm{KCl} 4 \mathrm{mM}, \mathrm{MgCl}_{2} 0.8 \mathrm{mM}, \mathrm{CaCl}_{2} 1.4 \mathrm{mM}, \mathrm{Na}_{2} \mathrm{HPO}_{4} 1.2 \mathrm{mM}$, $\mathrm{NaH}_{2} \mathrm{PO}_{4} 0.3 \mathrm{mM}$ and Dextrose 5mM, pH adjusted to 7.4) (Leggas et al., 2004c).

\subsubsection{Probe recovery}

Determination of probe recovery is critical for success of microdialysis. As the perfusion fluid continuously flows through the probe, the concentration equilibrium will never be achieved. Only a fraction of the drug concentration in the tissue is extracted in the probe. This faction is defined as the probe recovery, and many factors affect this recovery.

First, the length and diameter of probes (the functional part) significantly affect the probe recovery. The shorter or smaller probes give lower recovery simply because the perfusion fluid is allowed less time to exchange molecules with tissue fluid. For studies in mice, the probes are usually constructed in a very small size. For example, the probes used in this work are only $1 \mathrm{~mm}$ in length and $0.22 \mathrm{~mm}$ in diameter (MD-2211 Bioanalytical systems). Second, the perfusion flow rate also affects the recovery. The higher the flow rate the lower the recovery. In order to obtain enough recovery for microdialysis studies in CNS the flow rate can go as low as $0.5 \mathrm{ul} / \mathrm{min}$. Third, in vivo microenvironment influences the probe recovery significantly. Because the external media could differ considerably from tissue microenvironment, calibration in vitro does not predict the probe performance in vivo. For CNS studies, the probe recoveries could have significant variations in different experiments (de Lange et al., 2000).

Several methods are available to calibrate probe recovery in vivo (de Lange et al., 1997; de Lange et al., 2000). For example, no-net flux is time consuming, which is not realistic in our study. Internal standard real time method requires extra analysis approach. We will only discuss the method used in this work, the retrodialysis method. The idea 
was first described by Stahle and Wang (Stahle, 1994; Wang et al., 1993). If a drug solution with known concentration is perfused through the probe in vivo at the same flow rate as in the experiment, a fraction of drug will then be delivered to the tissue. It was demonstrated that this delivered fraction was equal to the extracted fraction when the drug was in the tissue (Stahle, 1994; Wang et al., 1993). The drug concentrations in the perfusion solution $\left(\mathrm{C}_{\text {in }}\right)$ and in the solution exiting the probe $\left(\mathrm{C}_{\text {out }}\right)$ can be measured before or after experiment. The recovery of the probe can then be calculated as

$$
R=\frac{C_{\text {in }}-C_{\text {out }}}{C_{\text {in }}} .
$$

\subsubsection{Pharmacokinetic considerations}

Based on the assumption that only the protein unbound drug is available for transport, a simple model (Figure 1-4) can be constructed to describe the drug transport between plasma and brain (Hammarlund-Udenaes et al., 1997; Wong et al., 1993).

The free drug concentration in plasma and brain ECF or ventricular CSF can be described by the following set of differential equations:

$$
\begin{aligned}
& V_{\text {plasma }} * \frac{d C_{\text {plasma }}}{d t}=R_{\text {inf }}-\left(C L+C L_{\text {in }}\right) * C_{u, \text { plasma }}+C L_{\text {out }} * C_{u, \text { brain }} \\
& V_{\text {brain }} * \frac{d C_{\text {brain }}}{d t}=C L_{\text {in }} * C_{u, \text { plasma }}-C L_{\text {out }} * C_{u, \text { brain }}
\end{aligned}
$$

where $\mathrm{V}_{\text {plasma }}$ is the volume of distribution of the free drug in the plasma compartment $\left(\mathrm{L} / \mathrm{m}^{2}\right), \mathrm{V}_{\text {brain }}$ is the apparent volume of distribution of the free drug in the brain $\left(\mathrm{L} / \mathrm{m}^{2}\right)$, $\mathrm{CL}$ is the total clearance of the drug from plasma $\left(\mathrm{L} / \mathrm{h} / \mathrm{m}^{2}\right), \mathrm{CL}_{\text {in }}$ and $\mathrm{CL}_{\text {out }}$ are intercompartmental clearance between plasma and brain $\left(\mathrm{L} / \mathrm{h} / \mathrm{m}^{2}\right), \mathrm{R}_{\text {inf }}$ is the constant infusion rate $\left(\mu \mathrm{g} / \mathrm{h} / \mathrm{m}^{2}\right)$. Integrating equation (1-2) from time zero to infinity and rearranging, we can obtain $\left(\mathrm{C}_{\text {brain }}=0\right.$ at time zero and infinity):

$$
\frac{C L_{\text {in }}}{C L_{\text {out }}}=\frac{A U C_{\text {brain }}^{0-\infty}}{A U C_{\text {plasma }}^{0-\infty}}
$$

where $A U C_{\text {brain }}^{0-\infty}$ is the area under the concentration-time curve of brain ECF or ventricular CSF drug integrated from time zero to infinity, $A U C_{p l a s m a}^{0-\infty}$ is the area under the concentration-time curve of plasma unbound drug integrated from time zero to infinity. 


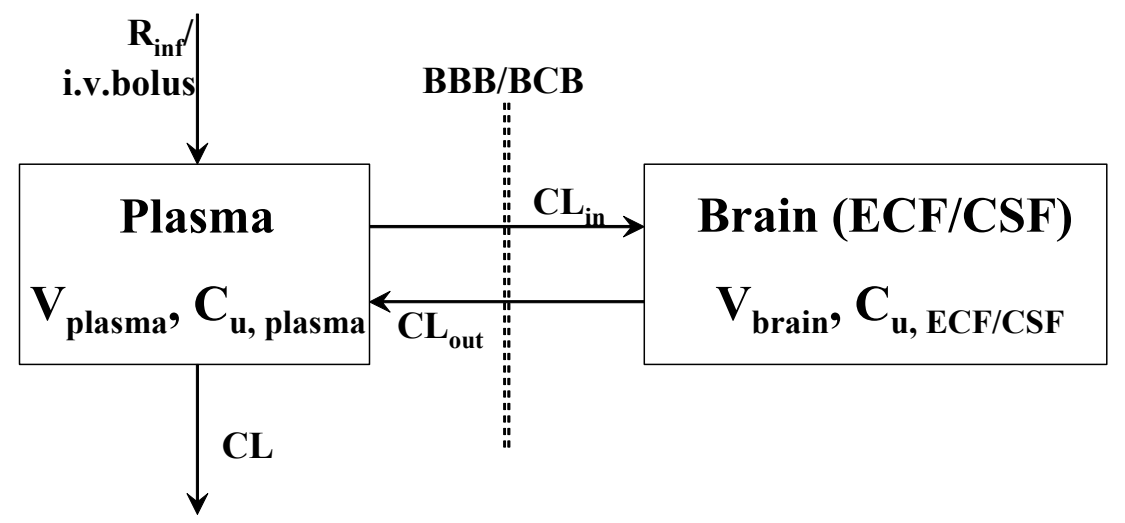

Figure 1-4 General two-compartment model for drug transport between plasma and brain.

$\mathrm{V}_{\text {plasma }}$ is the volume of distribution of the free drug in the body $\left(\mathrm{L} / \mathrm{m}^{2}\right), \mathrm{V}_{\text {brain }}$ is the apparent volume of distribution of the free drug in the brain $\left(\mathrm{L} / \mathrm{m}^{2}\right), \mathrm{C}_{\mathrm{u}}$, plasma is the protein unbound drug concentration in the plasma $(\mu \mathrm{g} / \mathrm{ml}), \mathrm{C}_{\mathrm{u}, \mathrm{ECF} / \mathrm{CSF}}$ is the protein unbound drug concentration in the brain (ECF or CSF) $(\mu \mathrm{g} / \mathrm{ml})$. CL is the total clearance of the drug from plasma $\left(\mathrm{L} / \mathrm{h} / \mathrm{m}^{2}\right), \mathrm{CL}_{\mathrm{in}}$ and $\mathrm{CL}_{\text {out }}$ are intercompartmental clearance between plasma and brain $\left(\mathrm{L} / \mathrm{h} / \mathrm{m}^{2}\right), \mathrm{R}_{\mathrm{inf}}$ is the constant infusion rate $\left(\mu \mathrm{g} / \mathrm{h} / \mathrm{m}^{2}\right)$. 
The clearance ratio $\mathrm{CL}_{\text {in }} / \mathrm{CL}_{\text {out }}$ can serve as a general indicator for drug transport activities across $\mathrm{BBB}$ or $\mathrm{BCB}$. Despite requirement of advanced pharmacokinetic modeling, this ratio could be obtained through compartmental analysis. Equation (1-3) converts the clearance ratio to the AUC ratio, which can be easily obtained through noncompartmental analysis. This ratio has several interesting properties (Deguchi, 2002).

When $\frac{A U C_{\text {brain }}^{0-\infty}}{A U C_{\text {plasma }}^{0-\infty}}<1$, active efflux transport from brain to blood is the most likely explanation (Hammarlund-Udenaes et al., 1997; Wong et al., 1993). However care must be taken because this AUC ratio reflects the overall drug clearance across the BBB or $\mathrm{BCB}$. If the drug is heavily metabolized or eliminated through bulk CSF route the ratio will also be significantly lower than unity. Many drugs fall into this category including alovudine, morphine, colchicine and topotecan etc. (Stahle and Borg, 2000; Xie et al., 1999; Desrayaud et al., 1997; Zhuang et al., 2006).

When $\frac{A U C_{\text {brain }}^{0-\infty}}{A U C_{\text {plasma }}^{0-\infty}}=1$, passive diffusion (including non-energy dependent facilitated transport) is the most likely explanation. Drugs like codeine and cocaine are the typical examples in this category (Xie and Hammarlund-Udenaes, 1998; Hedaya and Pan, 1997).

When $\frac{A U C_{\text {brain }}^{0-\infty}}{A U C_{\text {plasma }}^{0-\infty}}>1$, active influx transport from blood to brain is the most likely explanation. Although very few drugs has been shown to have such a property, the brain to plasma AUC ratio of unbound oxycodone was estimated to 3.0, which strongly suggested this drug had an active influx transport mechanism (Bostrom et al., 2006).

By calculating this AUC ratio it is possible to quantify the drug transport mechanisms across the $\mathrm{BBB}$ or the $\mathrm{BCB}$. One thing worth mentioning is that the AUC ratio is based on the assumption that only unbound drug is available for transport. Thus the total drug concentrations measured in plasma must be corrected with a protein unbound fraction $\left(f_{u}\right)$ to obtain the unbound drug concentrations before further analysis.

\subsubsection{Modulation of ABC transporters by 4-anilinoquinazolines}

The 4-anilinoquinazolines (4-AQ) were originally developed as compounds to inhibit receptor tyrosine kinases (RTK) such as epidermal growth factor receptor (EGFR/Erbbl) and Bcr-Abl etc. (Wakeling et al., 1996). The mechanism of such inhibition was demonstrated to be mediated through competing for ATP binding site of the receptors (Denny et al., 1996). Currently there are several 4-anilinoquinazolines approved by FDA, including gefitinib (Iressa ${ }^{\mathrm{TM}}, \mathrm{ZD} 1839$ ), erlotinib (Tarceva ${ }^{\mathrm{TM}}$, OSI774), lapatinib (Tykerb $\left.{ }^{\mathrm{TM}}, \mathrm{GW} 572016\right)$, imatinib (Gleevec ${ }^{\mathrm{TM}}$, STI-571), dasatinib $\left(\right.$ Sprycel $^{\mathrm{TM}}$, BMS-354825) and vandetanib (Zactima $\left.{ }^{\mathrm{TM}}, \mathrm{ZD} 6474\right)$. 
Stewart and colleagues first observed gefitinib showed minimal activity against a panel of 10 pediatric tumor xenografts that do not express the ERBBI receptor. However, combined with irinotecan, significantly greater than additive activity was observed in four of eight tumor models that expressed BCRP. The hypothesis that gefitinib inhibited BCRP was supported by an in vitro study in which gefitinib potently reverse resistance to SN-38 only in cell lines overexpressing functional BCRP (Stewart et al., 2004b). Similar results were also reported by another group. Yanase et al. demonstrated gefitinib reversed SN-38 resistance in BCRP-transduced human myelogenous leukemia K562 (K562/BCRP) and BCRP-transduced murine lymphocytic leukemia P388 (P388/BCRP) cells (Yanase et al., 2004).

Further studies have shown gefitinib not only strongly inhibited BCRP but also directly inhibited P-gp (Kitazaki et al., 2005; Nakamura et al., 2005; Yang et al., 2005). These results suggested a combination of gefitinib and camptothecin analogs could be clinically effective in $\mathrm{ABC}$ transporter overexpressing tumors. Leggas and colleagues demonstrated gefitinib modulated the function of Bcrp and Mdrl in vivo by using targeted mutation mouse models. Gefitinib increased oral absorption and decreased systemic clearance of topotecan in both $B c r p^{(--)}$and $M d r l a / b^{(--)}$mice (Leggas et al., 2006). work.

Based on these results, gefitinib was used as an $\mathrm{ABC}$ transporter modulator in this

\subsection{Blood brain tumor barrier (BTB)}

Although brain tumors still present some features of normal BBB, the pathological conditions significantly alter the BBB properties (Baluk et al., 2005). To distinguish from normal $\mathrm{BBB}$, the brain tumor $\mathrm{BBB}$ is referred to as blood brain tumor barrier (BTB). As we have discussed the properties of normal BBB, this section we will focus on the properties of BTB under pathological conditions.

\subsubsection{The factors affecting BTB}

\subsubsection{Disrupted tight junctions}

As mentioned in section 1.3.1, cerebral capillary endothelial cells are joined by tight junctions, these structures are clearly disrupted in BTB. Tight junction assembly requires multiple proteins including occludin, claudins, ZO-1, catenin, E-cadherin and actin etc (Wolburg and Lippoldt, 2002). Some of the proteins are missing or downregulated in brain tumor vessels.

Sawada et al. reported the ZO-1 protein expression was reduced in 26 human astrocytomas compared with normal brain tissue by using immunohistochemistry (Sawada et al., 2000). Leibner and colleagues screened the expression of several junction 
proteins in human glioblastoma multiforme. Claudin-1 was lost in the majority of tumor vessels and claudin-5 and occludin were significantly down-regulated in hyperplastic vessels (Liebner et al., 2000). Papadopoulos et al. studied the occludin expression in astrocytomas in different grades. It was demonstrated the immunostaining was inversely correlated with the tumor grades (Papadopoulos et al., 2001). A transmission electron microscopy study directly showed the open tight junctions in microvessels of human astrocytomas (Arismendi-Morillo and Castellano, 2005). These studies suggest tumor vasculature is structurally deficient in molecules that are crucial to maintain the BBB integrity.

\subsubsection{Abnormal morphology of blood vessels}

In addition to the disregulated expression of tight junction proteins, the tumor blood vessels are usually torturous and irregular (Baluk et al., 2005). As shown in Figure 1-5 a scanning electron micrograph, the luminal surface of normal blood vessel was smooth and covered by tight endothelial cells. In contrast in a pancreatic islet tumor, the blood vessel was rough and irregular and the lumen was significantly smaller. In addition to endothelial cells, other components of blood vessels including pericytes or muscle cells and basement membrane are all abnormal (Baluk et al., 2005) in tumor. Consequently the irregular blood vessels tend to reduce the blood flow to the tumor(Carmeliet and Jain, 2000; Baluk et al., 2005; Hashizume et al., 2000). It is believed that the observed features of tumor blood vessel prevail in all solid tumors.

\subsubsection{Angiogenesis related events}

While blood vessel growth is usually quiescent in adults, the tumor growth requires neo-angiogenesis (Folkman, 1971). Vascular endothelial growth factor (VEGF) has been identified as the most potent and critical angiogenic factor (Ferrara, 2004). VEGF expression is kept on a low level in normal tissues but greatly increased in brain tumor tissues. Weindel and colleagues analyzed VEGF expression level in 26 brain tumor surgical specimens (Weindel et al., 1994). PCR analysis revealed the VEGF mRNA level in malignant gliomas was increased 20 to 50-fold compared with low-grade tumors. In addition, using a radioreceptor assay it was possible to detect high VEGF-like activity in the cyst fluids of brain tumors, indicating the accumulation of VEGF and other permeability factors in brain tumor cysts (Weindel et al., 1994). These results were corroborated by Strugar and colleagues who found VEGF expression was well correlated with peritumoral brain edema status, which suggested VEGF expression increased the blood vessel permeability (Strugar et al., 1995). In addition to direct expression of VEGF, other pro-angiogenic mechanisms were also activated. Nabors and colleagues have shown that HuR, an mRNA stabilizer, is consistently expressed in malignant brain tumors. HuR was further demonstrated to stabilize cytokines such as VEGF, Cyclooxygenase-2, IL-8 and TGF- $\beta$ etc., which are known to be involved in angiogenesis (Nabors et al., 2001).

A consequence of active angiogenesis is the increased activity of basement membrane metabolism (Kalluri, 2003). Basement membrane, a specialized extra-cellular 


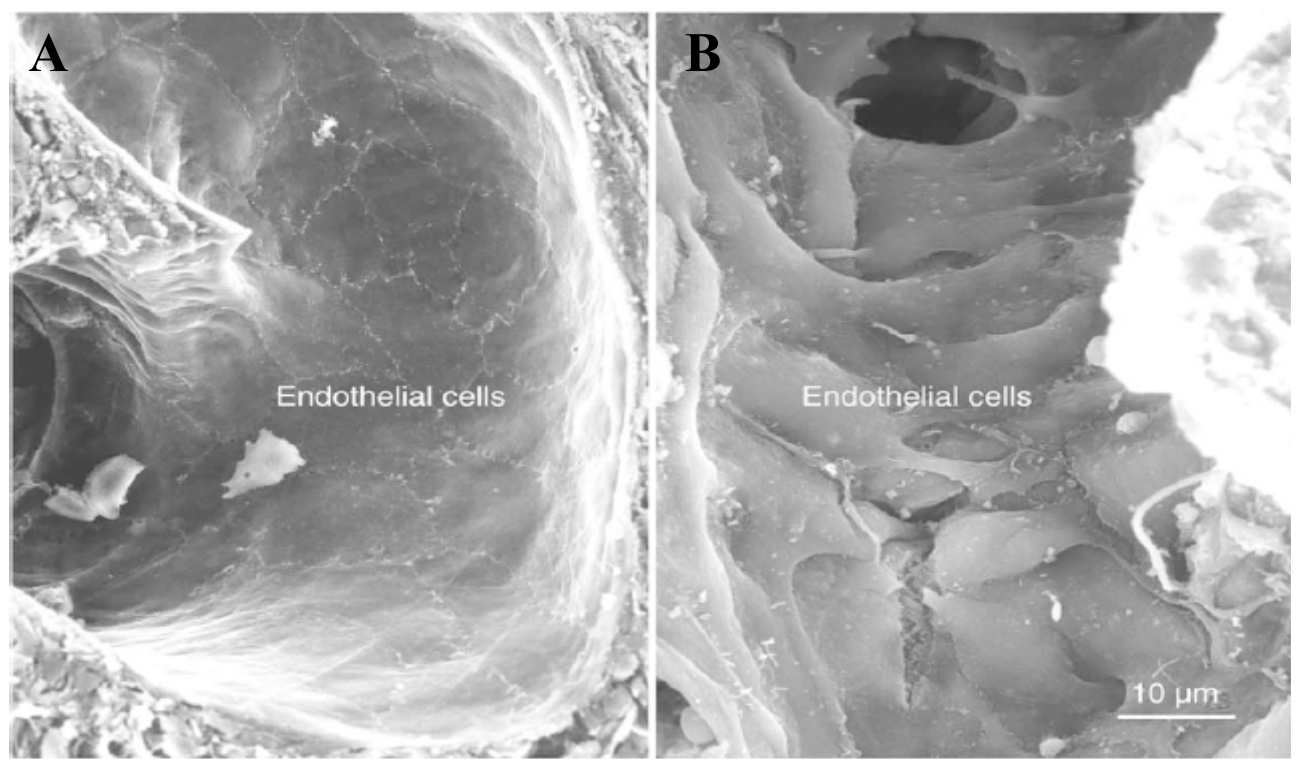

\section{Figure 1-5 Blood endothelial cells in normal or tumor tissues.}

A) Capillary endothelial cells in normal pancreatic tissue. B) Capillary endothelial cells in pancreatic cancer tissue

Source: Reprinted with permission. Baluk P, Hashizume H, McDonald DM, 2005. Cellular abnormalities of blood vessels as targets in cancer. Curr. Opin. Genet. Dev. 15: 102-111. 
matrix, is a structural and functional component of the integrated BBB. About 50 proteins make up the basement membrane, $50 \%$ of which is composed of collagen (especially type IV) (Kalluri, 2003). Other components include nidogen, laminin, and perlecan. These proteins are highly crosslinked in an organized way to support the vascular endothelial cells. For angiogenesis to occur from the existing blood vessels the basement membrane has to be degraded first. The degradation process is also highly regulated with multiple enzymes involved such as matrix metalloproteinases (MMPs) and collagenases (Arroyo et al., 2007; Rundhaug, 2005). The basement membrane degradation generates many fragments from the structural components, which have either pro or anti-angiogenic effects (Kalluri, 2003; O'Reilly et al., 1994; Colorado et al., 2000; Kamphaus et al., 2000; O'Reilly et al., 1997; Maeshima et al., 2002; Hamano et al., 2003; Carmeliet and Jain, 2000). With tumor growth, basement membrane is degraded and reconstructed through the process of tumor induced neo-angiogenesis. All these angiogenetic activities affect the permeability of blood vessel. Due to its complexity, the overall influence on drug penetration is unpredictable.

\subsubsection{Other molecular events}

In normal $\mathrm{BBB}$, the vesicular transport activity is very low. However this low transport mechanism could be activated in BTB due to the disregulation of certain molecular events. Early studies by Black and colleagues have shown that bradykinin and leukotriene C4 (LTC4) increased molecular transport across the blood-tumor barrier (BTB) (Hashizume and Black, 2002). It was also demonstrated that this increased transport was not mediated through tight junction opening but rather through enhanced vesicular transport (Hashizume and Black, 2002). The authors further reported that the overexpressed calcium-dependent potassium $\left(\mathrm{K}_{\mathrm{Ca}}\right)$ channels and the ATP-sensitive potassium $\left(\mathrm{K}_{\mathrm{ATP}}\right)$ channel in tumor capillaries were responsible for the enhanced transport activity (Ningaraj et al., 2003; Ningaraj et al., 2002). These data suggest another approach is needed for specific drug delivery to brain tumors.

\subsubsection{Early studies on drug penetration in the brain tumors}

Reliable drug penetration data in brain tumors are very limited. Early studies mainly focused on the construction of various brain tumor models and measurement of blood vessel permeability by using autoradiography. These studies demonstrated the BTB was generally more permeable than the normal BBB (Groothuis et al., 1982; Hasegawa et al., 1983; Shapiro and Shapiro, 1986; Shapiro, 1985; Groothuis et al., 1984).

The early studies on drug CNS penetration mainly focused on methotrexate. Slordal et al. reported a normal brain to plasma methotrexate concentration ratio of 0.2 in rats (Slordal et al., 1988). Nierenberg et al. reported greater methotrexate concentrations in and near brain tumor than in ventricular CSF or serum in human adults (Nierenberg et al., 1991). However the brain tissue homogenate analysis neither discriminated the drug in extracellular fluid from intracellular fluid, nor separated the free drug from the protein 
bound drug. The true penetration of methotrexate into the brain/brain tumor could be overestimated.

\subsubsection{In vivo microdialysis studies on drug penetration through BTB}

A few studies using a microdialysis approach demonstrated methotrexate penetration into brain tumors were much lower than determined by homogenization method in rats. With an i.v. infusion of methotrexate at $75 \mathrm{mg} / \mathrm{kg}$, de lange et al. reported that the brain tumor to plasma $\mathrm{AUC}$ ratio $\left(\mathrm{AUC}_{\text {brain }} / \mathrm{AUC}_{\text {plasma }}\right)$ was 0.12 compared with normal brain to plasma ratio 0.05 (de Lange et al., 1995). However in this study the brain tumor model was constructed from R-6 rhabdomyosarcoma instead of a brain tumor line and the microdialysis probe was calibrated in vitro, which left some uncertainty for the accuracy of the results.

According to Groothuis an animal brain tumor model should meet two criteria: 1) the tumor should originally be a brain tumor and 2) the tumor proliferation and histological grading should be predictable and reproducible (Groothuis et al., 1983). With these criteria Devineni et al. constructed a rat glioma model (RG-2) to study methotrexate brain tumor penetration (Devineni et al., 1996a). Although $\mathrm{AUC}_{\text {brain }} / \mathrm{AUC}_{\text {plasma }}$ was similar (0.05) in tumor and normal brain, the absolute AUC value in tumor was much higher $(584 \mu \mathrm{g} \cdot \mathrm{min} / \mathrm{ml})$ than normal brain $(172 \mu \mathrm{g} \cdot \mathrm{min} / \mathrm{ml})$. However the drug clearance and distribution volume were significantly lower in tumor bearing rats than normal ones. The authors attributed it to the loss of fluid volume in tumor bearing animals. Further pharmacokinetic modeling analysis showed the mass transfer coefficient across the BTB was much greater $\left(8.9 \times 10^{-4} \mathrm{ml} / \mathrm{min}\right)$ than the BBB $\left(2.75 \times 10^{-4} \mathrm{ml} / \mathrm{min}\right)$. This study suggested the methotrexate had a higher penetration rate in brain tumor than normal brain tissue.

Dukic et al. investigated methotrexate penetration in two different rat glioma models. CNS1 presented a proliferation rate twice as much as C6 did in vivo. As a result of which, the CNS1 had more active neo-angiogenesis activity than C6. After a fast i.v. infusion of methotrexate $50 \mathrm{mg} / \mathrm{kg}$, brain drug concentrations were determined by cerebral microdialysis. The final $\mathrm{AUC}_{\text {brain }} / \mathrm{AUC}_{\text {plasma }}$ ratio in $\mathrm{CNS} 1$ model was lower than in $\mathrm{C} 6$ model. In addition, the in vivo recoveries in tumor tissue were significantly different between the two tumor models. These results suggested the drug penetration not only depended on the presence of the tumor but also on the tumor model itself (Dukic et al., 2004).

Nakashima et al. determined the cisplatin distribution in a 9L rat glioma model. Cisplatin $(3.5 \mathrm{mg} / \mathrm{kg})$ was administered as a selective intracarotid infusion for $30 \mathrm{~min}$. Blood samples were collected through femoral vein and cerebral microdialysis samples were collected every $10 \mathrm{~min}$ for $120 \mathrm{~min}$. The cisplatin plasma protein unbound fraction was also estimated. With this setting the brain to plasma AUC ratio of unbound cisplatin was determined to be 0.69 in tumor tissue and 0.04 in normal brain (Nakashima et al., 
1997). The study provides an example that CNS penetration of a hydrophilic drug (e.g. cisplatin) can be significantly increased in tumor tissue compared with normal brain.

Most recently, Appraraju et al. reported a pyrimidine nucleoside analogue, gemcitabine, also had a higher penetration in a C6 rat glioma model. Following an i.v. bolus injection of gemcitabine $(25 \mathrm{mg} / \mathrm{kg})$, microdialysis samples were collected $15 \mathrm{~min}$ pose-dose and then every $30 \mathrm{~min}$; blood samples were collected periodically over 8 hours. The brain to plasma AUC ratio was determined 0.19 in tumor tissue and 0.09 in normal brain (Apparaju et al., 2008).

In summary, the in vivo microdialysis studies provided invaluable data, which indicated the BTB penetration for anticancer drugs varied significantly. The drug penetration depended on drug physiochemical properties, the tumor models, and the location of the tumor. Although these results suggested the drug penetration through the BTB was increased in comparison with that in the normal brain, they should not be extrapolated without caution.

\subsection{Summary}

The outcome for patients with malignant CNS tumors is currently very poor. Chemotherapy is an important treatment modality, however the exact role of chemotherapy for CNS tumor treatment is not clear. Where novel agents such as camptothecin analogs have demonstrated potent activity in preclinical studies, the results from clinical trials were quite disappointing. Although such a discrepancy could be attributed to inherent tumor insensitivity, it is also likely to be associated with inadequate drug delivery due to the presence of the $\mathrm{BBB}$ and the $\mathrm{BCB}$.

While the specialized structures of the BBB and the BCB protect the brain from either endogenous or xenobiotic toxins, they also limit the penetration of anti cancer drugs (e.g. camptothecin analogs). The fact that the $\mathrm{ABC}$ transporters are expressed at the $\mathrm{BBB}$ and the $\mathrm{BCB}$ provides an opportunity to modulate the permeability of the two membrane barrier systems. Although camptothecin analogs were substrates for multiple $\mathrm{ABC}$ transporters (e.g. P-gp, BCRP, and MRP4 etc.) in vitro, their in vivo role has not yet been confirmed. When microdialysis sampling technique is utilized in targeted mutation mouse models, the role of the $\mathrm{ABC}$ transporters in vivo can be better defined.

Pathological conditions significantly alter the BTB properties; as a result drug penetration through the BTB could differ from the normal BBB. It is of clinical interest to understand how camptothecin analogs penetrate through the BTB. In vivo microdialysis technique is an invaluable tool to address this question. 


\subsection{Objectives}

The major focus of this work was to define the in vivo role of two ABC transporters Bcrp and P-gp in determination of topotecan (a model drug of camptothecin analogs) penetration through $\mathrm{BBB}$ and $\mathrm{BCB}$.

However in order to accurately measure the drug concentration in CSF, the microdialysis probe has to be precisely implanted into mouse lateral ventricle. The conventional method doesn't offer a reliable approach. Thus the first objective is to develop a reliable surgical procedure to implant a microdialysis probe into mouse lateral ventricle.

The second objective was to determine the topotecan penetration in vCSF and ECF of brain parenchymal tissue by utilizing cerebral microdialysis in several knockout

mouse models including Mdrla/b ${ }^{(-/)}, B_{c r p 1} 1^{(-/)}$and Mdrla/b/Bcrpl $1^{(-/)}$. Pharmacokinetic models were developed to describe the data.

With clear understanding of mechanisms of topotecan CNS penetration under normal physiological conditions in vivo we extended our investigation to topotecan penetration across BTB in an animal tumor models. The third objective is to determine the topotecan penetration in a human glioma (U-87) xenograft mouse model. 


\section{Chapter 2. A Modified Surgical Procedure for Microdialysis Probe Implantation in the Lateral Ventricle of an FVB Mouse ${ }^{*}$}

\subsection{Introduction}

Microdialysis has been widely used to sample endogenous biochemicals (e.g., neurotransmitters) or drugs in many different tissues in situ. It has many advantages over common extraction methods such as whole tissue homogenization. These advantages include continuous sampling without fluid loss, measurement of discrete anatomic compartments, clean samples suited for direct bioanalysis without prior processing, and measurement of unbound or pharmacologically active moieties(Benveniste and Huttemeier, 1990). This technique has been well established in multiple studies evaluating the penetration of unbound drug in numerous tissues (Boschi and Scherrmann, 2000; Bito et al., 1966; Meirieu et al., 1986; Lehmann, 1989; Arner et al., 1988; Ben-Nun et al., 1988).

Microdialysis has been particularly useful for studying drug distribution in the central nervous system (CNS) including ventricular cerebrospinal fluid (CSF) and extracellular fluid (ECF) of brain parenchymal tissue. Our previous studies have demonstrated that the camptothecin analog, topotecan, has differential penetration into CSF and ECF of the FVB mouse model (Zhuang et al., 2006). The results of our studies have shown that the brain ECF to plasma area under the concentration-time curve (AUC) ratio of unbound topotecan lactone was 0.2 compared with the ventricular CSF to plasma AUC ratio which was approximately 1.2 (Zhuang et al., 2006). These results emphasize the importance of having the ability to distinguish between separate anatomic compartments such as the penetration of a drug into the CSF versus the ECF. Microdialysis affords this unique advantage which when combined with sensitive and specific analytical methods provide an accurate and quantitative assessment of the CNS penetration of a drug.

Although CNS drug penetration studies could be performed in larger animal models such as the rat, dog, or nonhuman primate, the murine models may be manipulated genetically in ways that are not possible in the larger models. This provides an important tool to address mechanistic questions such as those related to drug transport by making use of transporter knock-out mouse models (Jonker et al., 2002; Jonker et al., 2005; Schinkel et al., 1997) and transporter modulators (e.g., gefitinib). Our first studies in the mouse model used previously published surgical procedures that were designed to primarily to assess drug penetration into brain ECF only. We adapted those techniques to assess topotecan penetration in both ventricular CSF and brain ECF. Although these techniques were successful, they were technically very challenging and required large numbers of animals to get the required data. Moreover, we found slight anatomical differences between strains of mice. Therefore, we needed a more robust, reliable, and

\footnotetext{
* Reprinted by permission. Shen J, Fraga C, Calabrese C, McCarville MB, Schaiquevich P, Stewart CF, 2008. A modified surgical procedure for microdialysis probe implantation in the lateral ventricle of a FVB mouse. J. Pharm. Sci.
} 
reproducible technique specifically for the FVB mouse model in order to enhance our studies of drug penetration through the $\mathrm{BCB}$. Thus, the objectives of the present study were to develop a surgical procedure to implant a microdialysis probe into the lateral ventricle of FVB mice, to verify probe position through imaging technology and review of histology, and finally to validate the procedure experimentally by assessing topotecan CSF penetration in the FVB mouse model alone and in the presence of a transporter modulator such as gefitinib.

\subsection{Materials and methods}

\subsubsection{Drugs}

Topotecan (Hycamtin, GlaxoSmithKline, Philadelphia, PA) was prepared in sterile water at a concentration of $1 \mathrm{mg} / \mathrm{ml}$. Gefitinib tablets (Iressa, AstraZeneca), containing $250 \mathrm{mg}$ gefitinib per tablet, were pulverized. Gefitinib powder was wetted with $0.5 \%$ Tween $20(20 \% \mathrm{v} / \mathrm{v}$ final volume $)$ and suspended in carboxymethylcellulose $(0.25 \% \mathrm{w} / \mathrm{v})$ to a final concentration of $40 \mathrm{mg} / \mathrm{mL}$.

\subsubsection{Animals}

FVB female mice (Taconic, Germantown, NY) weighing 20-25g were used for this study. The mice were maintained on a 12 hour light/dark cycle with free access to food and water. After surgery, the mice were housed individually in cages. All procedures were approved by St. Jude Institutional Animal Care and Use Committee and in accordance with the Association for Assessment and Accreditation of Laboratory Animal Care (AALAC).

\subsubsection{Stereotaxic coordinates calculation and adjustment}

To define the axes, $\mathrm{X}$ was described as medial/lateral (left+/right-) movements along a horizontal line perpendicular to the midline on the skull. Y was set as anterior $(+) /$ posterior(-) movements along the line parallel to the midline of the brain. Finally, $Z$ was set as dorsal(+)/ventral(-) vertical movements into the brain. Although this study was performed in FVB female mice (20-25g), our initial coordinates were based on the C57BL/J6 strain as it was the only mouse strain with published stereotaxic coordinates in the brain (Franklin, 1997).

First, we selected a region that included coronal sections from bregma $-0.1 \mathrm{~mm}$ to bregma $-0.58 \mathrm{~mm}$, and measured the $\mathrm{Z}$ coordinates of the roof and bottom of the right

lateral ventricle. The coordinates were then plotted on a chart with dimensions $\mathrm{Y}$ and $\mathrm{Z}$ as shown in Figure 2-1A, where $X$ is $1 \mathrm{~mm}$ lateral to the midline. Second, a right triangle was constructed to represent the ventricle geometrically. Based on the triangle, a 
marginal angle $\alpha$ could be calculated where the angle $\alpha=\beta=\gamma$. Therefore, the $\tan (\gamma)=0.48 / 1.6=0.3$, and $\gamma=\tan ^{-1}(0.3)=16.7$ degree. From these calculations, it was possible to theorize that at any angle greater than 16.7 degree, the probe should be in the lateral ventricle. An angle of 20 degrees was chosen empirically to calculate the $\mathrm{Y}$ and $\mathrm{Z}$ coordinates on the skull surface for use during cranial surgery. The coordinates were calculated as $Y=$ reference- $1.3 \mathrm{~mm}, \mathrm{Z}=$ reference $-2.1 \mathrm{~mm}$, as shown in Figure 2-1B ( $X=$ reference- $1 \mathrm{~mm}$ as mentioned above and angle $\alpha=20$ degree). The reference was the corresponding coordinate of the bregma point.

\subsubsection{Cranial surgery}

The general surgical procedure has been previously described in detail (Leggas et al., 2004c). Briefly, the mouse was anesthetized with ketamine (Abbott Laboratories, Chicago IL)/xylazine (Butler Company, Columbus, OH). A guide cannula with an inner stylet (MD-2255, Bioanalytical Systems, West Lafayette, IN) was positioned in the lateral ventricle. Mice were allowed to recover for 3-5 days prior to insertion of the microdialysis probe (MD-2211, Bioanalytical Systems, West Lafayette, IN). Animals were euthanized at the conclusion of each study. The head was removed and immersed in $10 \%$ neutral buffered formalin (NBF) for fixation prior to histologic evaluation or micro-CT. The stereotaxic coordinates were slightly adjusted and refined accordingly for subsequent studies based upon information gained from imaging techniques and histology.

\subsubsection{Ultrasound imaging}

Mice bearing the implanted microdialysis probe were anesthetized deeply with isoflurane $3-4 \%$ and maintained with a nose cone. Anesthesia was supplemented with intraperitoneal injections of ketamine/xylazine $0.2 \mathrm{~mL} / 100 \mathrm{gm}$ as needed. The state of anesthesia was assessed periodically by "pinch" test of the rear paw for reflex response. The hair was shaved and the skin treated with betadine solution. A coronal incision was made and the skin retracted dorsally to expose the skull. The bone was etched and removed to create a "window" exposing the occipital region of the brain. The skin flap was pulled forward and re-attached along the original incision with surgical cement. Creation of this cranial "window" allows for direct imaging of the brain tissue with an ultrasound probe.

A VisualSonics (Toronto, ON) Vevo 770 ultrasound machine and mouse imaging platform and monitoring system were used. After anesthesia, the mouse was placed in a prone position on the imaging platform with the chin extended. The $70855 \mathrm{MHz}$ highresolution, linear, transducer was secured in position over the skull vertex so that the lateral ventricles were visualized in the coronal plane (Figure 2-2A). We postulated that if the probe was properly positioned within the ventricular system, then injection of 
A

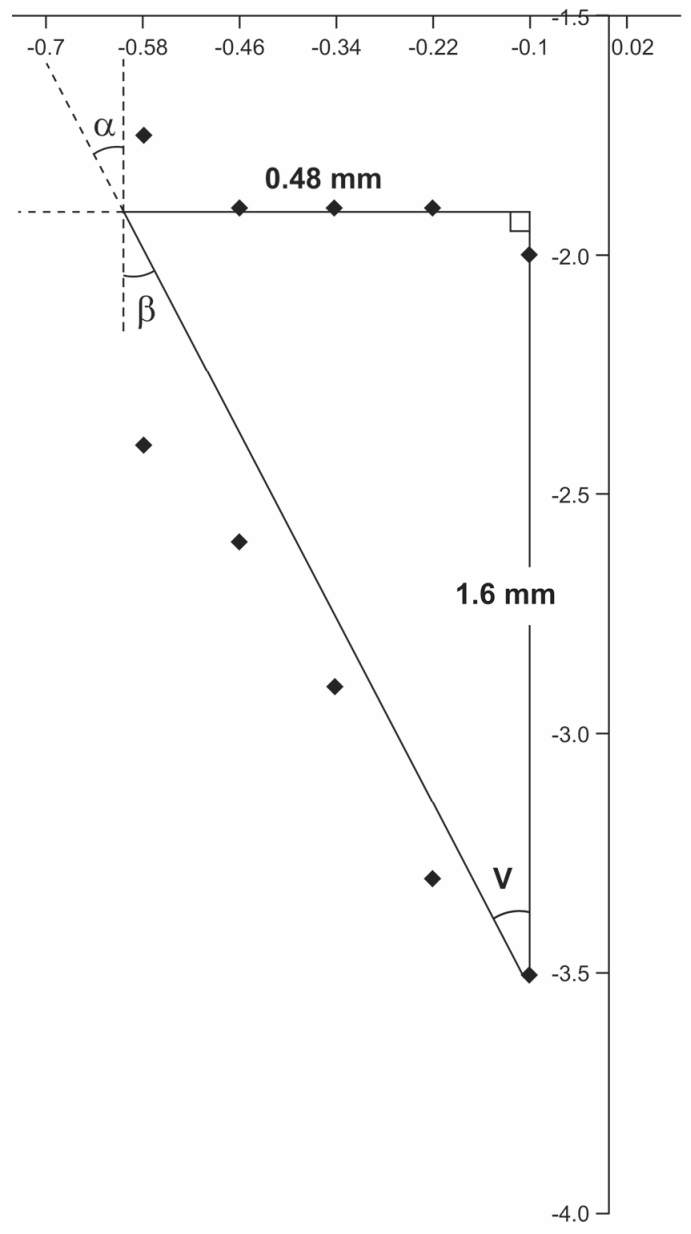

B

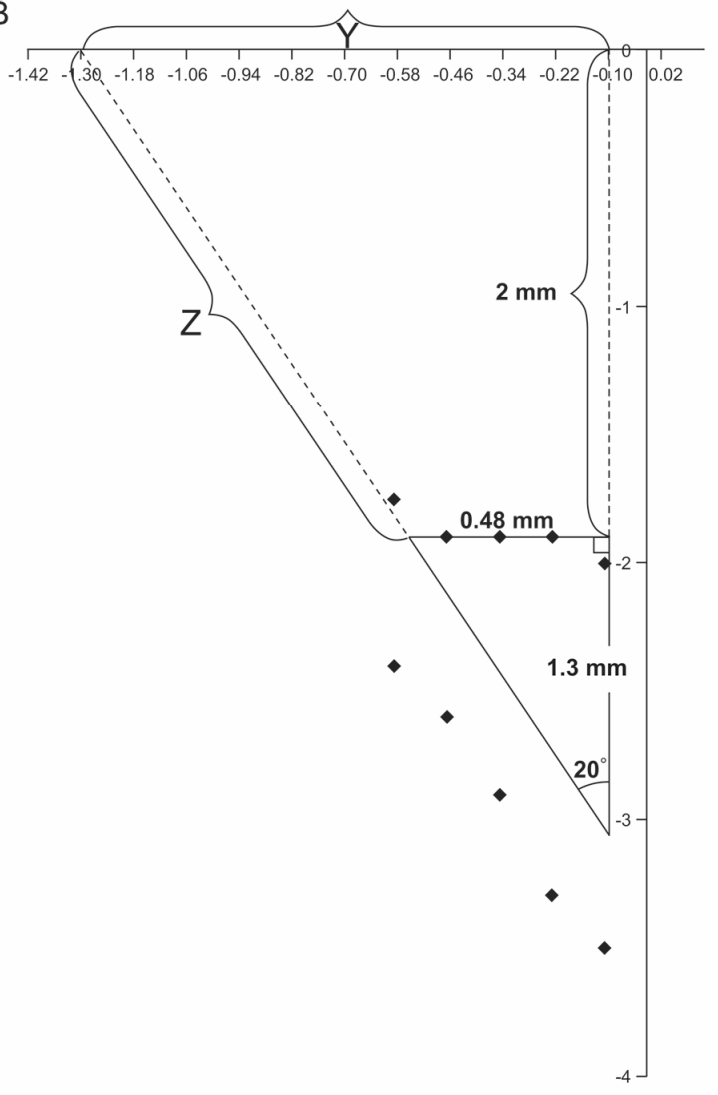

Figure 2-1 Schematic demonstration of stereotaxic coordinates calculation.

A). Lateral ventricle coordinates of C57BL/J6 male mouse (coronal sections containing bregma $-0.1 \mathrm{~mm}$ to bregma $-0.58 \mathrm{~mm}$ ) and right triangle model representing that part of the ventricle. indicate the positions of the roofs and bottoms of ventricles in the coronal sections. See text for calculation steps. (B). Calculation for the coordinates on the skull surface that can be used in surgical procedure. 


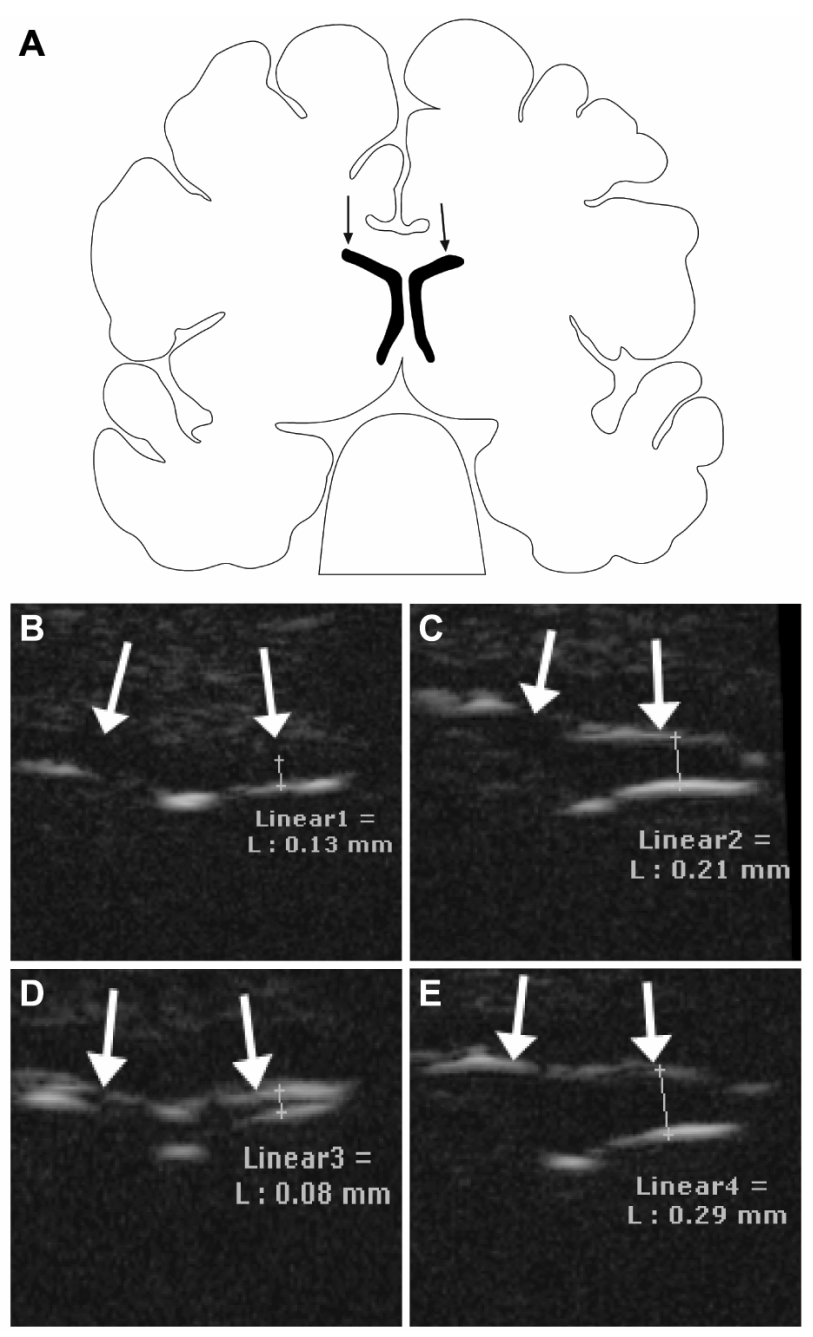

\section{Figure 2-2 Ultrasound imaging study.}

A) Horizontal diagram of brain showing lateral ventricles (arrows) (B-E) Coronal ultrasound images of a mouse bearing an intracranial probe obtained B) Before the injection of sterile saline into the intracranial probe cannula. Note the slit-like appearance of the normal, non-expanded lateral ventricles (arrows). The cranio-caudal diameter of the left lateral ventricle is $0.13 \mathrm{~mm}$. C) This image obtained immediately after the injection of $100 \mu \mathrm{L}$ of saline shows expansion of the lateral ventricles (arrows) to a cranio-caudal diameter of $0.21 \mathrm{~mm}$. D) This image obtained 5 minutes after the first injection shows decompression of the lateral ventricles (arrows) to a cranio-caudal diameter of 0.08 , suggesting circulation of the injected saline out of the lateral ventricles. E) This image obtained immediately after a second injection of $170 \mu \mathrm{L}$ of sterile saline, shows a greater degree of ventricular expansion (arrows) relative to that seen in Fig 2-2C, to a cranio-caudal diameter of $0.29 \mathrm{~mm}$. These findings were taken as evidence of proper probe position. 
sterile saline into the probe cannula would expand the ventricles and this change would be readily apparent on ultrasound imaging. Therefore, we obtained continuous real-time and static ultrasound imaging before, during, and after an initial injection of $100 \mu \mathrm{L}$ of sterile saline through the intracranial probe cannula followed 10 minutes later by a confirmatory, second injection of $170 \mu \mathrm{L}$ of sterile saline. To document a change in ventricular size we measured the cranio-caudal diameter of the mid-portion of the left lateral ventricle on pre and post-injection images.

\subsubsection{Micro-CT analysis}

All micro-CT analyses were performed using an Explore LocusSP specimen scanner (GE Healthcare, Piscataway, NJ). Briefly, mice were euthanized and the microdialysis probe was removed. MicroFil solution (Flow tech Inc., Carver, Massachusetts) was prepared in advance by mixing 2 parts of MV-diluent with one part MV compound. MicroFil solution (100-150 $\mu$ l) was injected through the guide cannula with a 27 gauge needle syringe. Once the injection was complete, the microdialysis probe was immediately re-inserted into the brain through the guide cannula. The MicroFil compound was allowed to solidify for 30 minutes at room temperature, and then the whole mouse head was removed and immersion fixed in 10\% NBF for at least 24 hours. Before scanning skulls were suspended in $70 \%$ ethanol and immobilized using sponges.

For intact skull imaging, specimens were analyzed at $80 \mathrm{kVp}$ and $70 \mathrm{~mA}$ giving 400 total views and an isotropic resolution of $28 \mu \mathrm{m}$. After whole skull scanning and reconstruction, the cannula and probe were removed and the brain extracted. The probe was then reintroduced into the brain and tissue immobilized as before in $70 \%$ ethanol. In the case of excised brain imaging, a second scan protocol was designed that would allow soft tissue discrimination but retain the ability to resolve radio-opaque materials such as MicroFil. This protocol included reduction in voltage to $55 \mathrm{kVp}$ and increase in current to $118 \mathrm{~mA}$. In order to limit artifact noise during scanning, an average of 8 individual frames was used for each view. By performing a $360^{\circ}$ scan, 900 total views were obtained and an isotropic resolution of $16 \mu \mathrm{m}$ achieved.

\subsubsection{Histology}

As mentioned previously, at the conclusion of a microdialysis experiment, the animal was euthanized and the brain removed and fixed in 10\% NBF for at least 24 hours. The brain was then grossed by cutting into saggital segments, dehydrated, cleared and embedded in a paraffin block. Sections were cut on a microtome at $4 \mu \mathrm{m}$ thickness and every tenth section was collected and stained with haematoxylin and eosin. All sections from a given block were examined microscopically to verify the location of the microdialysis probe via the track in the tissue (Zhuang et al., 2006). 


\subsubsection{Microdialysis study in vCSF of FVB mice}

The details of the microdialysis procedure were previously reported (Zhuang et al., 2006; Hammarlund-Udenaes et al., 1997). Briefly on the day of an experiment, a microdialysis probe (MD-2211, Bioanalytical Systems, West Lafayette, IN) was flushed with artificial CSF (aCSF) and inserted through the cannula into the brain lateral ventricle of a FVB mouse. The aCSF perfusion rate was set as $0.5 \mu \mathrm{l} / \mathrm{min}$. The probe was allowed to equilibrate in vivo for 1 hour. Topotecan was administered by i.v. bolus through the lateral tail vein at a dosage of $4 \mathrm{mg} / \mathrm{kg}$ (Zhuang et al., 2006). Gefitinib was administered by oral gavage at a single dose of $200 \mathrm{mg} / \mathrm{kg}$ one hour before intravenous topotecan. The dialysate samples were directly loaded onto a sample loop $(2 \mu \mathrm{l})$ and analyzed through an online microbore HPLC system (Zhuang et al., 2006; Leggas et al., 2004c). During the microdialysis period, three plasma samples were collected from each mouse at $0.25,1$ and 3 hours after topotecan administration. The samples, which were processed and analyzed for topotecan lactone using a previously published HPLC assay with fluorescence detection, were then used to determine the topotecan disposition in that mouse as previously described (Zhuang et al., 2006).

\subsubsection{Microdialysis probe recovery}

At the completion of the microdialysis study, when the topotecan CSF concentration was undetectable, a two-hour washing period was allowed. Then the probe recovery was determined using an in vivo retrodialysis technique. Previously in vitro recovery studies from our lab have shown that topotecan lactone and carboxylate have the same recovery (unpublished data); therefore, we used recovery of total topotecan to represent the recovery for each form of topotecan. A topotecan solution $(50 \mathrm{ng} / \mathrm{ml})$ was prepared in artificial CSF and the total topotecan concentration $\left(\mathrm{C}_{\mathrm{in}}\right)$ was determined by HPLC. The solution was perfused $(0.5 \mu \mathrm{l} / \mathrm{min})$ through the microdialysis probe and the first four measurements exiting the probe were averaged as $C_{\text {out }}$. The recovery $\left(R_{\text {topotecan }}\right)$ was estimated as shown in Equation 2-1:

$$
R_{\text {topotecan }}=\left(C_{\text {in }}-C_{\text {out }}\right) / C_{\text {in }}
$$

\subsubsection{Noncompartmental analysis of vCSF pharmacokinetic data}

The pharmacokinetic analysis of topotecan lactone was performed for each mouse (Zhuang et al., 2006). Topotecan lactone concentrations in vCSF were corrected for the hydrolysis of topotecan lactone to the carboxylate that occurs in $\mathrm{pH} 7.4$ artificial CSF as described previously (Leggas et al., 2004c). The AUC in vCSF from time 0 to infinity was estimated using the linear trapezoidal method with the addition of a residual area as depicted in Equation 2-2, 


$$
A U C_{\text {brain }}^{0 \rightarrow \infty}=\sum_{i=1}^{n} \frac{C_{i}+C_{i-1}}{2} * \Delta t+C_{n} / \beta
$$

where $\mathrm{Ci}$ is the topotecan concentration in dialysate; $\Delta \mathrm{t}$ is the sample injection interval; $\mathrm{Cn}$ is the last measurable topotecan concentration; $\beta$ is estimated by linear regression of the logarithm of the last 4-5 measurable concentrations.

\subsubsection{Compartmental analysis of plasma pharmacokinetic data}

Plasma topotecan lactone concentration-time data were first corrected for plasma protein binding using an unbound factor determined previously (i.e., 0.36) (Zhuang et al., 2006). A two-compartment pharmacokinetic model was fitted to the plasma topotecan lactone concentration-time data using maximum a posteriori probability (MAP) Bayesian estimation as implemented in ADAPT II (D'Argenio and Schumitzky, 1979). Pharmacokinetic parameters determined included volume of distribution of central compartment $(\mathrm{Vc})$, elimination rate constant $(\mathrm{ke})$, and intercompartmental rate constants. Using these parameters, the unbound topotecan lactone concentrations in the plasma were simulated in ADAPT II. The area under the concentration-time curve from zero to infinity $\left(\mathrm{AUC}_{0 \rightarrow \infty}\right)$ for the unbound topotecan plasma was calculated by integration of the simulated concentration-time data from model estimates. A ratio of the vCSF AUC to unbound plasma topotecan lactone AUC was calculated and used as a measurement of topotecan vCSF penetration (Hammarlund-Udenaes et al., 1997).

\subsubsection{Statistical analysis}

Statistical analysis was performed in Sigma Stat 3.1. The student's t-test was used to compare the results of modified procedure with those of conventional approach. The difference was considered significant when $\mathrm{p}<0.05$.

\subsection{Results}

\subsubsection{Determination of stereotaxic coordinates}

We first used the calculated coordinates $(X=$ reference- $1 \mathrm{~mm}, \mathrm{Y}=$ reference- $1.3 \mathrm{~mm}$, $\mathrm{Z}=$ reference- $2.1 \mathrm{~mm}$, Angle $\alpha=20$ degree) to implant cannulas/probes in the right lateral ventricle of FVB female mice (see Methods for details of calculation of coordinates). The postmortem histology showed the probe missed the ventricle on the $Y$ axis by $0.5-0.7 \mathrm{~mm}$ (Figure 2-3A); however, the $\mathrm{X}, \mathrm{Z}$, and angle $\alpha$ were acceptable. We attributed this to differences in strains between FVB and C57BL/J6. We then adjusted the Y coordinate for subsequent studies. With an adjusted Y coordinate, the probe was determined to be precisely located in the lateral ventricle (Figure 2-3B). The final stereotaxic coordinates were determined as $X=$ reference- $1 \mathrm{~mm}, Y=$ reference- $0.8 \mathrm{~mm}, Z=$ reference- $2.1 \mathrm{~mm}$ with an 

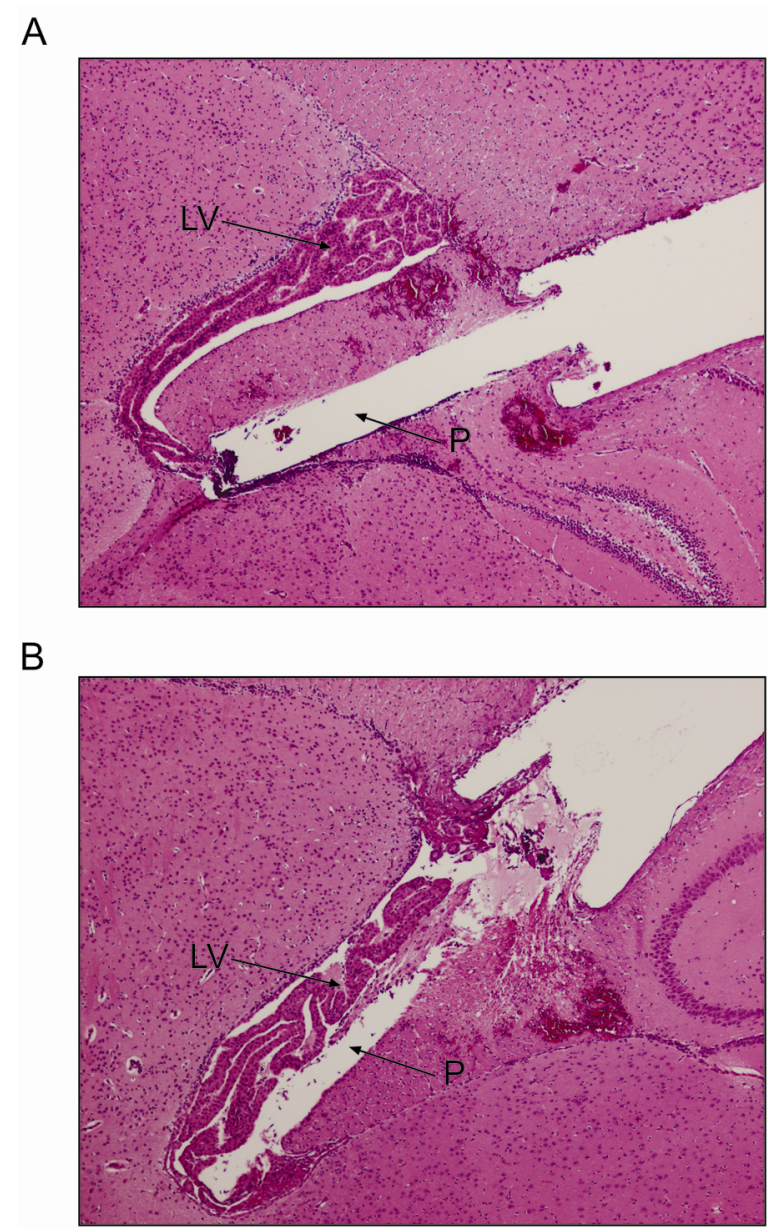

\section{Figure 2-3 H\&E staining of saggital brain section after probe insertion.}

(A), microdialysis probe track (P) missing the lateral ventricle (LV) by using calculated coordinates. (B), microdialysis probe track within the lateral ventricle after coordinates adjusted. 
angle $\alpha=20$ degree leaning toward posterior. The reference is the corresponding coordinate of the bregma point.

\subsubsection{Ultrasound imaging study}

Due to the very small size of the probe, we were unable to confidently visualize the exact location relative to the lateral ventricles on the pre-injection ultrasound images (Figure 2-2B). However, during the first injection of saline, we observed an immediate expansion of the lateral ventricles that was readily apparent on ultrasound imaging and confirmed by an increase in the cranio-caudal diameter from $0.13 \mathrm{~mm}$ pre-injection (Figure 2-2B) to $0.21 \mathrm{~mm}$ post-injection (Figure 2-2C). Five minutes after the first injection, the lateral ventricles were smaller, measuring $0.08 \mathrm{~mm}$ in diameter, (Figure 22D) suggesting circulation of the saline out of the lateral ventricles. During the second injection, the ventricles again immediately expanded, this time to a greater degree than after the initial, smaller volume, injection, to a diameter of $0.29 \mathrm{~mm}$ (Figure 2-2E). These imaging results were interpreted as evidence of proper placement of the probe into the lateral ventricle.

\subsubsection{Micro-CT imaging analysis}

In order to capture a direct image of the cannula/probe in the ventricle, a high resolution micro-CT analysis was performed. To confirm placement of the probe in the lateral ventricle, radio-opaque polymer MicroFil was infused through the implanted cannula. The location of the polymer, cannula, and probe was determined using micro$\mathrm{CT}$. The tip of the probe was located in the lateral ventricle with the whole head scan in both coronal (Figure 2-4A) and saggital (Figure 2-4B) planes. By imaging the excised brain (Figures 2-4C and 2-4D), in the absence of artifacts introduced by the metal screws implanted to secure the cannula, produced a superior to demonstrate the relative position of the probe in the right lateral ventricle.

\subsubsection{Topotecan penetration through blood CSF barrier in FVB mice}

With the coordinates determined and probe placement verified through imaging techniques (ultrasound and micro-CT) and histologic review, this modified surgical procedure was applied to placement of microdialysis probes for the study of topotecan penetration into vCSF. During a baseline period prior implementation of this modified technique, our previous technique for probe placement was utilized. Of 20 microdialysis studies conducted during this period results from seven studies were interpretable (based on histological verification) for a success rate of approximately $35 \%$. However, with implementation of the modified surgical procedure for microdialysis probe implantation, results from 14 of the next 20 studies were interpretable for a success rate of $70 \%$. 

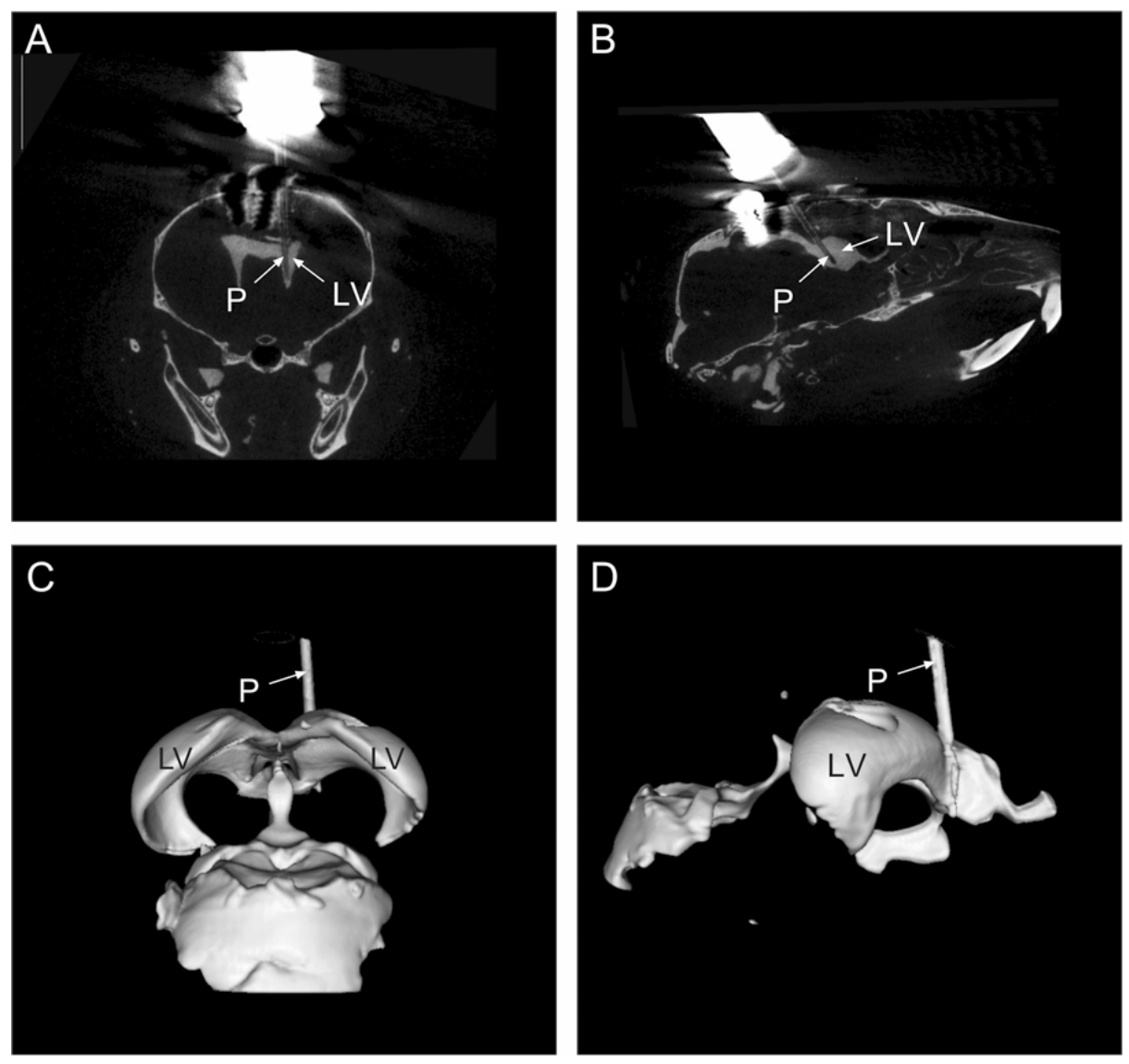

Figure 2-4 MicroCT analysis of intact head and excised brain following MicroFil infusion.

For the whole head scan (coronal plane (A) and saggital plane (B)) the coronal slice (A) is orientated in plane with the microdialysis probe to show the insertion path. The excised brain scan shows posterior (C) and lateral (D) views. $\mathrm{LV}=$ Lateral Ventricle; $\mathrm{P}=$ Probe. 
A noncompartmental approach was used to directly calculate the unbound topotecan lactone AUC from the vCSF microdialysis data. A two-compartment pharmacokinetic model was fitted to the unbound topotecan lactone plasma concentrations to estimate the pharmacokinetic parameters (Zhuang et al., 2006; D'Argenio and Schumitzky, 1979). Based upon these pharmacokinetic parameters, the plasma concentrations were then simulated using ADAPT II (D'Argenio and Schumitzky, 1979) and the plasma AUC was calculated using a log-linear trapezoidal approach. In contrast to previously published results, the unbound topotecan (TPT) lactone concentrations measured in vCSF were much higher with this modified surgical procedure. Interestingly, lower unbound TPT lactone concentrations in VCSF in several experiments were observed, which were comparable to what was previously reported. However, after completion of histologic examination and analysis of CT results for these specific experiments, it was noted that the probe was not completely located within the lateral ventricle. In experiments where the probe was confirmed in the lateral ventricle, the vCSF to plasma AUC ratio of unbound TPT lactone $\left(\mathrm{AUC}_{\mathrm{u}, \mathrm{CSF}} / \mathrm{AUC}_{\mathrm{u} \text {,plasma }}\right)$ was calculated as $2.3 \pm 0.11$ (see Table 2-1), which was almost twice as high as previously determined $\left(\mathrm{AUC}_{\mathrm{u}, \mathrm{CSF}} / \mathrm{AUC}_{\mathrm{u} \text {,plasma }}\right.$ 1.2) with the conventional approach (Zhuang et al., 2006). The in vivo probe recoveries in vCSF were estimated $8.07 \pm 2.42 \%$.

The effect of gefitinib modulation on topotecan vCSF penetration was also investigated with this modified procedure. The vCSF to plasma AUC ratio of unbound TPT lactone was then determined as $1.02 \pm 0.03$ (see Table 1), which was comparable to previously reported data $\left(\mathrm{AUC}_{\mathrm{u}, \mathrm{CSF}} / \mathrm{AUC}_{\mathrm{u}, \text { plasma }}\right.$ approximately 0.98$)$ (Zhuang et al., 2006). The in vivo probe recoveries were $6.7 \pm 1.0 \%$.

\subsection{Discussion}

In this study, we developed a modified surgical procedure to implant a microdialysis probe into the lateral ventricle of FVB mice. Utilizing stereotaxic coordinates calculated from $\mathrm{C} 57 \mathrm{BL} / \mathrm{J} 6$ as our starting point, we have refined those coordinates and changed the angle of the probe placement. This improved surgical procedure takes advantage of the width of the posterior region of the lateral ventricle and gains more space on the Z-axis (Figure 2-1B). This resulted in greatly increased accuracy and precision of microdialysis cannula/probe insertion into the ventricle. To determine if these modifications would impact our results, several microdialysis studies of topotecan in ventricular CSF were performed. The results of these studies showed that when the microdialysis probe was consistently placed in the lateral ventricular space the topotecan ventricular CSF to plasma AUC ratio was greater than that observed with the conventional technique.

To gain insight into the CSF penetration of a compound, studies are often conducted in larger animal species (e.g., nonhuman primate (Blaney et al., 1993; Blaney et al., 1998; D'Argenio and Schumitzky, 1979)), primarily because CSF samples can be collected directly via ventricular shunts or reservoirs. Although accurate placement of ventricular probes requires skill and technique, it can be done relatively easy compared 
Table 2-1 vCSF to plasma AUC ratios of unbound topotecan lactone at the presence or absence of gefitinib.

\begin{tabular}{ccc}
\hline Treatment & Animal ID & AUC ratio \\
\hline & $\# 1$ & 2.22 \\
& $\# 2$ & 2.32 \\
Topotecan 4mg/kg & $\# 3$ & 2.18 \\
& $\# 4$ & 2.45 \\
& $\# 5$ & 2.30 \\
& $\# 6$ & 2.45 \\
& Average & $2.32 \pm 0.11^{*}$ \\
Topotecan 4mg/kg+gefitinib & & \\
$200 \mathrm{mg} / \mathrm{kg}$ & $\# 7$ & 1.05 \\
& $\# 9$ & 1.01 \\
& Average & 0.99 \\
& & $1.02 \pm 0.03 * *$ \\
\hline
\end{tabular}

$*$ Mean \pm SD, $\mathrm{n}=6$

$* *$ Mean \pm SD, $\mathrm{n}=3$

$\mathrm{P}<0.05$ (Student's t test) 
with smaller animal species. However, small animal models (e.g., mice) present a significant technical challenge because of the extremely small size of their ventricles and CSF volume (Cirrito et al., 2003).

In order to investigate the drug penetration through the blood CSF barrier (BCB) utilizing microdialysis, it is crucial to insert the probe directly into the lateral ventricle. Upon examination of coronal sections in the anterior region of the mouse lateral ventricle, it is relatively deep $(1 \mathrm{~mm})$ but narrow $(\sim 0.3 \mathrm{~mm})$, and the posterior portion is relatively wide $(0.8-1 \mathrm{~mm})$ but shallow $(0.5-0.8 \mathrm{~mm})$. The microdialysis probe itself is $1 \mathrm{~mm}$ in length and $0.22 \mathrm{~mm}$ in diameter. Smaller probes are not practical as the recovery is usually low. Due to the dimensional limitations in the mouse, it is extremely difficult to precisely implant a probe into the ventricle using the conventional perpendicular insertion method. Moreover, it requires extensive technical expertise and is labor intensive requiring many experiments to collect the appropriate data. Many times after histologic review, data from experiments are excluded when the probe is determined to have penetrated the ventricle or not deep enough. Thus the objective of the present study was to develop and validate a modified method to implant a microdialysis probe at an angle and from a more dorsal insertion point into the lateral ventricle of a mouse.

This modified procedure also has application for ventricular drug delivery in mouse models. Although, methods for intracerebral injection of drugs in mice were first described by Haley and McCormick (Haley and Mccormick, 1957) and modified by Clark (Clark et al., 1968) and Boschi (Boschi et al., 1981), due to similar reasons as discussed above, the procedures lack accuracy and reliability. If the conventional perpendicular targeting method is used, the width advantage (posterior part of the ventricle) is sacrificed for depth (anterior part of the ventricle). This modified procedure could help deliver drugs into the ventricle much more accurately.

Although this study was initiated to develop a more reliable and robust surgical procedure, unexpectedly the observation that higher unbound topotecan lactone concentrations were present in the vCSF suggests the modified procedure positions the probe for superior performance than the conventional perpendicular method.

Consequently the vCSF to plasma AUC ratio of unbound topotecan lactone was almost doubled $(2.3 \pm 0.11)$ with the modified procedure over that previously reported $(1.2 \pm 0.06)$ with the conventional perpendicular method. Interestingly when mice were pretreated with gefitinib the AUC ratios obtained with the modified procedure (1.02 \pm 0.03$)$ were comparable to those obtained with the conventional method $(0.98 \pm 0.05)$ (Zhuang et al., 2006). Gefitinib has been reported to modulate ATP-binding cassette (ABC) transporters and inhibiting active transport of their substrates (e.g. topotecan, irinotecan) (Leggas et al., 2006; Stewart et al., 2004b). This laboratory previously reported the expression of two ABC transporters, breast cancer resistance protein (Bcrp1) and P-glycoprotein (P-gp) on the apical side of choroid plexus epithelial cells in FVB mice (Zhuang et al., 2006). Based on the results of this study the modified surgical procedure demonstrated enhanced positioning of the microdialysis probe that affords quantitation of active drug transport superior to the conventional perpendicular method. However when gefitinib is present, the active drug transport was inhibited. When this occurs only passive diffusion is 
permitted, and as would be expected, both methods give similar values, $\left(\mathrm{AUC}_{\mathrm{u}, \mathrm{CSF}} / \mathrm{AUC}_{\mathrm{u}, \mathrm{plasma}}\right.$ around 1.08). Therefore the underestimation of drug penetration in vCSF could be caused by either partial probe contact of vCSF (puncture through the ventricle) or non-optimal position of the probe in lateral ventricle (against the wall of the ventricle).

Although it is not exactly clear why this modified procedure more accurately quantitates active CSF drug efflux, it is likely related to the choroid plexus distribution in the lateral ventricle and the direction of CSF circulation in the ventricular system. The choroid plexus is not evenly distributed through the whole lateral ventricle, but is dense in the posterior region. CSF is secreted by the choroid plexus located in lateral ventricles and "percolates" down to the third and fourth ventricle, and then eventually out of ventricular system (Johanson et al., 2005). This modified procedure orients the probe in the posterior part of lateral ventricle, which contains dense segments of choroid plexus and also is in the direct route of the CSF as it flows down to the third ventricle. In contrast, the conventional perpendicular method places the probe in the anterior region of the ventricle, which is not only narrow but also contains much less choroid plexus. Additionally, this positioning is not in the route of CSF flow, which is in a rostral/caudal direction.

Due to possible strain differences, the calculated coordinates based on C57BL/J6 mice may not be directly applied to other mouse strains. This report demonstrated refinement of the coordinates for the FVB strain. The optimal coordinates for a specific mouse model should be experimentally optimized by either postmortem histologic inspection or by imaging technology. In this case, Y coordinate for FVB female mice was adjusted by $0.5-0.7 \mathrm{~mm}$. However the calculated coordinates are invaluable as an initial reference point. Finally we propose that this modified procedure may be used for microdialysis experiments investigating the presence of a compound (e.g., neurotransmitters) or drug penetration into the vCSF in mice. 


\section{Chapter 3. Penetration of Topotecan Lactone across Blood-Brain Barrier and Blood-CSF Barrier: Differential Role of P-Glycoprotein (P-gp) and Breast Cancer Resistance Protein (BCRP)}

\subsection{Introduction}

Treatment of patients with primary central nervous system (CNS) tumors continues to challenge both physicians and researchers. The overall survival rate for these patients has not significantly improved over the last three decades (Jemal et al., 2007). Although surgical resection and radiotherapy are the cornerstones of therapy, chemotherapy is an important treatment modality for many patients. For example, in children less than three years old chemotherapy is preferred to avoid or delay the use of radiotherapy (Rutkowski et al., 2005). However, despite aggressive chemotherapy with currently available agents, clinical outcomes are far from satisfactory (Motl et al., 2006) and development of new chemotherapeutic agents is urgently demanded.

One promising class of drugs, the camptothecin analogs (e.g., topotecan and irinotecan) have demonstrated potent antitumor activity when tested in a panel of human brain tumor xenograft mouse models (Houghton et al., 1995; Hare et al., 1997). However, disappointing results from clinical trials using these chemotherapeutic agents in patients with primary CNS tumors suggest that their in vivo brain penetration might be constrained by the presence of blood brain barrier (BBB) and blood cerebrospinal fluid (CSF) barrier (BCB) (Friedman et al., 1999b; Chamberlain, 2002; Cloughesy et al., 2002; Blaney et al., 1996; Stewart et al., 2004a; Bernier-Chastagner et al., 2005; Chintagumpala et al., 2006). The proteins of the ATP-binding cassette (ABC) transporter family are known to be expressed on the apical side of cerebral vascular endothelial cells, the major component of BBB (Cooray et al., 2002; Leggas et al., 2004a; Schinkel et al., 1994), and may play a significant role on the restriction of topotecan penetration into the brain, as recently shown (de Vries et al., 2007; Leggas et al., 2004a). In fact, camptothecins are substrates of multiple ABC transporters (Sparreboom et al., 2003; Gottesman et al., 2002; Loscher and Potschka, 2005a) and several in vitro studies have demonstrated that overexpression of human breast cancer resistance protein (Bcrp/ABCG2), P-glycoprotein (P-gp), or multidrug resistance protein 4 (MRP4) conferred resistance to topotecan (Yang et al., 1995; Maliepaard et al., 1999a; Tian et al., 2006; Hendricks et al., 1992b).

We have reported that MRP4 (ABCC4) is expressed on the basolateral side of the choroid plexus epithelial cells in both humans and rodents (Leggas et al., 2004a). Taking advantage of an in vivo cerebral microdialysis technique that allows to measure unbound topotecan concentrations in selected tissues or anatomical compartments, we showed increased topotecan concentrations in CSF of Mrp4 knockout mice than wild type (Leggas et al., 2004a). This result suggests Mrp4 constrained topotecan penetration into CSF. However our further study in FVB wild type mouse demonstrated unbound topotecan lactone concentration in CSF was actually higher than that in plasma (Zhuang et al., 2006). The expression of Mrp4 cannot explain the observation. These data strongly 
suggest that at least one other drug efflux pump exists to actively move topotecan from the blood into the CSF.

We reported that murine Bcrp1 and P-gp were expressed on the apical side of the epithelial cells of choroid plexus (BCB) (Zhuang et al., 2006). As topotecan is also a substrate for Bcrp1 and P-gp (Yang et al., 1995; Hendricks et al., 1992b) it is highly probable that Bcrp1 and P-gp are together responsible for the high penetration of topotecan across the BCB. However in vivo data to support this hypothesis is lacking.

Most recently Tellingen et al. reported P-gp and Bcrp1 working together in limiting the brain penetration of topotecan by using a brain homogenate method (de Vries et al., 2007). However the study did not distinguish the CSF compartment from the extracellular fluid (ECF) of brain parenchymal tissue. Furthermore the homogenate method may not explain the drug transport mechanisms across the BBB as it mixes the protein bound and unbound drug from brain microvessels, extracellular fluid, and intracellular fluid.

A complete characterization of the differential function of $\mathrm{BBB}$ versus $\mathrm{BCB}$ in the topotecan brain pharmacokinetics could be of clinical relevance. For instance, it is reported that medulloblastoma patients have a better response rate as compared to glioma patients in several clinical trials with topotecan (Stewart et al., 2004a; Bernier-Chastagner et al., 2005; Chintagumpala et al., 2006). A reasonable hypothesis would be that medulloblastoma, due to its neuraxis location, has more access to CSF regulated by BCB. Glioma is more parenchymal tissue based with very limited CSF access and mainly perfused through cerebral capillary regulated by BBB. Localized pharmacokinetic sampling in the knockout mouse brain could clarify the mechanisms that control the topotecan penetration through the BBB and the BCB, and such knowledge could lead to an effective modulation strategy to enhance drug CNS penetration. However, sampling the mouse brain is challenging and requires special skills and equipments.

To improve our understanding of the role of the $\mathrm{ABC}$ transporters in the in vivo CNS penetration of anticancer drugs, we aim at characterizing their differential function in the BBB and BCB barriers. Pursuing this goal, in the present study we take advantage of 1) knockout mouse models for Bcrp1 and P-gp, 2) the pharmacological effect of gefitinib as a modulator of the $\mathrm{ABC}$ transporters, and 3) a sampling technique (microdialysis) that allows the "online" characterization of the unbound concentrations of topotecan lactone and carboxylate in localized CNS compartments.

\subsection{Materials and methods}

\subsubsection{Drugs and chemicals}

Topotecan hydrochloride (Hycamtin, GlaxoSmithKline, Philadelphia, PA) for use in pharmacokinetic studies was prepared in sterile water $(1 \mathrm{mg} / \mathrm{ml})$. Gefitinib tablets 
(Iressa, AstraZeneca), containing $250 \mathrm{mg}$ gefitinib per tablet, were pulverized. Gefitinib powder was reconstituted with $0.5 \%$ Tween $20(20 \% \mathrm{v} / \mathrm{v}$ final volume $)$ and suspended in carboxymethylcellulose $(0.25 \% \mathrm{w} / \mathrm{v})$ to a final concentration of $40 \mathrm{mg} / \mathrm{mL}$. Topotecan solutions used for chromatography $(1 \mathrm{mg} / \mathrm{ml})$ were prepared in dimethyl sulfoxide (DMSO). All other solvents and chemicals and solvents used were analytical grade or better.

\subsubsection{Animals}

FVB, Bcrp1 $1^{(-)}$(Jonker et al., 2002), Mdrla/b ${ }^{(-)}$(Schinkel et al., 1997), and Mdrla $/ b^{(--)} B_{c r p I^{(--)}}$(Jonker et al., 2005) female mice weighing 20-25 g were ordered from Taconic, Germantown, NY. All targeted mutation mouse models are crossed on an FVB genetic background. The mice were maintained on a 12-hour light/dark cycle with free access to food and water. After surgery, the mice were housed individually in cages. All procedures were approved by St. Jude Institutional Animal Care and Use Committee and in accordance with the Association for Assessment and Accreditation of Laboratory Animal Care (AALAC).

\subsubsection{Topotecan plasma protein binding}

Topotecan lactone plasma protein binding was determined in plasma from each of the mouse models including FVB, BcrpI $1^{(-)}, M d r 1 a / b^{(-)}$, and Mdrla $/ b^{(-)} B c r p 1^{(-)}$. Topotecan lactone was added to plasma from each genotype to make a $500 \mathrm{ng} / \mathrm{ml}$ solution. Spiked plasma $(0.5 \mathrm{~mL})$ was added to a Centrifree YM-30 (Millipore Corporation, Billerica, MA) sample reservoir and centrifuged at $2000 \mathrm{~g}$ for 20 minutes. Topotecan was extracted from the protein and ultrafiltrate portion of the device, respectively, by adding $50 \mu \mathrm{L}$ of sample immediately to $200 \mu \mathrm{L}$ of cold methanol stored on dry ice. Samples were analyzed by an HPLC method as previously reported (Furman et al., 1996; Baker et al., 1996). Topotecan lactone fraction unbound was calculated as amount of unbound topotecan lactone divided by total amount of topotecan lactone in plasma.

\subsubsection{Brain surgery}

The procedure of brain surgery to implant a guide cannula for microdialysis study in vCSF (Shen et al., 2008) or ECF (Zhuang et al., 2006; Leggas et al., 2004c) has been previously reported. Prior to any surgery, the mouse was anesthetized with ketamine (Abbott Laboratories, Chicago IL) /xylazine (Butler Company, Columbus, OH). Briefly, for the vCSF microdialysis study, the guide cannula (MD-2255, Bioanalytical Systems, West Lafayette, IN) was inserted into the lateral ventricle at an angle of 20 degrees posterior $(1 \mathrm{~mm}$ lateral, $0.8 \mathrm{~mm}$ posterior, $2 \mathrm{~mm}$ ventral to the bregma point). For the ECF study, the guide cannula was inserted vertically into the striatum $(1.8 \mathrm{~mm}$ lateral, $0.6 \mathrm{~mm}$ 
anterior, and $2 \mathrm{~mm}$ ventral to the bregma point). After insertion of the cannula, a mouse was allowed three to five days to recover prior to any microdialysis experiment.

\subsubsection{Microdialysis in ventricular CSF and brain ECF}

The microdialysis procedure and probe calibration was previously reported in detail (Leggas et al., 2004c; Zhuang et al., 2006). Briefly, on the day of an experiment, a microdialysis probe (MD-2211, Bioanalytical Systems, West Lafayette, IN) was primed and flushed with blank artificial CSF (aCSF, $\mathrm{NaCl} 148 \mathrm{mM}, \mathrm{KCl} 4 \mathrm{mM}, \mathrm{MgCl}_{2} 0.8 \mathrm{mM}$, $\mathrm{CaCl}_{2} 1.4 \mathrm{mM}, \mathrm{Na}_{2} \mathrm{HPO}_{4} 1.2 \mathrm{mM}, \mathrm{NaH}_{2} \mathrm{PO}_{4} 0.3 \mathrm{mM}$ and Dextrose $5 \mathrm{mM}, \mathrm{pH}$ adjusted to 7.4). The probe was inserted through the cannula into the brain lateral ventricle or striatum, and the aCSF perfusion rate was set at $0.5 \mu 1 / \mathrm{min}$. The probe was allowed to equilibrate in vivo for 1 hour. Topotecan $(4 \mathrm{mg} / \mathrm{kg}$ ) was administered by i.v. bolus through the lateral tail vein. The dialysate samples were directly loaded onto a sample loop $(2 \mu \mathrm{l})$ and analyzed through an online microbore HPLC system (Leggas et al., 2004c). Probe recovery was determined using the in vivo retrodialysis method after each experiment was completed. During the microdialysis period, three plasma samples were collected from a mouse at $0.25,1$, and 3 hours after topotecan administration for plasma pharmacokinetic analysis (Zhuang et al., 2006). At the completion of the microdialysis experiment, the mouse was euthanized, the brain was fixed in 10\% neutral buffered formalin for 24 hours, and then embedded in paraffin. To locate the microdialysis probe track, haematoxylin and eosin stained sections $(4 \mu \mathrm{m})$ were examined microscopically.

\subsubsection{Microdialysis probe recovery}

Once the vCSF topotecan concentration was undetectable, a two-hour wash period was begun. At the end of the wash period, probe recovery was determined using the in vivo retrodialysis method with topotecan as calibrator. In vitro recovery studies done in our lab showed that topotecan lactone and carboxylate have the same recovery (unpublished data); therefore, we used recovery of total topotecan to represent the recovery for each form of topotecan. A topotecan solution $(50 \mathrm{ng} / \mathrm{ml})$ was prepared in artificial CSF and the total topotecan concentration (Cin) was determined by HPLC. The solution was perfused $(0.5 \mu \mathrm{l} / \mathrm{min})$ through the microdialysis probe and the first four measurements exiting the probe were averaged as Cout. The recovery $\left(R_{\text {topotecan }}\right)$ was estimated as shown in Equation 3-1:

$$
R_{\text {topotecan }}=\left(C_{\text {in }}-C_{\text {out }}\right) / C_{\text {in }}
$$

\subsubsection{Noncompartmental analysis of brain topotecan data}

Pharmacokinetic analysis was performed for each mouse (Zhuang et al., 2006). Topotecan carboxylate and lactone concentrations in vCSF were corrected for the hydrolysis of topotecan lactone in $\mathrm{pH} 7.4$ aCSF as described previously (Leggas et al., 
2004c). The AUC of lactone and carboxylate in vCSF or ECF from time 0 to infinity was estimated using the linear trapezoidal method with the addition of a residual area as depicted in Equation 3-2:

$$
A U C_{\text {brain }}^{0 \rightarrow \infty}=\sum_{i=1}^{n} \frac{C_{i}+C_{i-1}}{2} * \Delta t+C_{n} / \beta
$$

where $\mathrm{C}_{\mathrm{i}}$ is the topotecan concentration in the dialysate; $\Delta \mathrm{t}$ is the sample injection interval; $\mathrm{C}_{\mathrm{n}}$ is the last measurable topotecan concentration; $\beta$ is estimated by linear regression of the logarithm of the last 4-5 measurable concentrations.

\subsubsection{Topotecan plasma pharmacokinetics}

As with our previous murine microdialysis study (Zhuang et al., 2006), the goal of each plasma pharmacokinetic study was to assess the topotecan lactone systemic exposure. Because of logistical issues (e.g., blood volume), no more than three samples per mouse could be obtained, thus a limited sampling model was utilized to assess unbound topotecan lactone systemic exposure. Before this analysis could proceed, population mean and variances (i.e., population priors) for topotecan lactone pharmacokinetic parameter estimates were determined in each murine genotype model. A plasma pharmacokinetic study was performed for each genotype $\left(\mathrm{Bcrpl}^{(-/)}, \mathrm{Mdrla} / \mathrm{b}^{(-/)}\right.$, and $M d r l a / b^{(--)} B_{c r p l} I^{(--)}$) and treatment (topotecan $4 \mathrm{mg} / \mathrm{kg}$ i.v. alone or topotecan 4 $\mathrm{mg} / \mathrm{kg}$ i.v. plus oral gefitinib $200 \mathrm{mg} / \mathrm{kg}$ ) using the same approach previously described (Zhuang et al., 2006) to obtain the population mean and variance (estimated via NONMEM) for each pharmacokinetic parameter. Briefly, for each of these studies six to seven mice were used and each mouse was randomly sampled four times out of five sampling time points (e.g., 0.25, 0.5, 1.5, 3 and 6 hours) by either retro-orbital or cardiac puncture at the final time point. Sample processing and topotecan lactone bioanalysis have been previously reported in detail (Baker et al., 1996; Furman et al., 1996; Zhuang et al., 2006). In the studies where the effect of gefitinib was evaluated, it was administered by oral gavage at a dosage of $200 \mathrm{mg} / \mathrm{kg}$ one hour before topotecan, which was administered through lateral tail vein injection (Zhuang et al., 2006).

For each microdialysis experiment (e.g., topotecan with or without gefitinib pretreatment), a two-compartment pharmacokinetic model was fitted to the plasma topotecan lactone concentration-time data using maximum a posteriori probability (MAP) Bayesian estimation as implemented in ADAPT II (D'Argenio and Schumitzky, 1979). The population priors used for this MAP Bayesian analysis were determined for each genotype as described above. The pharmacokinetic parameters estimated included volume of central compartment $\left(\mathrm{V}_{\mathrm{c}}\right)$, systemic clearance $\left(\mathrm{CL}_{\mathrm{t}}\right)$, volume of peripheral compartment $\left(\mathrm{V}_{\mathrm{p}}\right)$, and intercompartmental clearance $\left(\mathrm{CL}_{\mathrm{p}}\right)$. Using these parameters, the unbound topotecan lactone concentrations in the plasma were simulated for each experiment using ADAPT II. The area under the plasma concentration-time curve from zero to infinity $\left(A U C_{0 \rightarrow \infty}\right)$ for the unbound topotecan lactone was calculated by integration of the simulated concentration-time data from the model estimates. 
The extent of CNS penetration of topotecan lactone was determined by the ratio of unbound topotecan lactone AUC in brain ECF or ventricular CSF to the unbound plasma topotecan lactone AUC (Hammarlund-Udenaes et al., 1997). Student's t-test was used to compare the unbound topotecan lactone brain to plasma AUC ratio after each treatment.

\subsubsection{Three-compartment analysis of combined plasma and brain lactone pharmacokinetic data}

The previous assessment of topotecan CNS penetration was a hybrid of noncompartmental and compartmental determinations, so a three compartment model (Hammarlund-Udenaes et al., 1997; Gupta et al., 2006; Zhuang et al., 2006) was developed to fit unbound topotecan lactone concentration-time data in brain parenchymal ECF or vCSF and plasma as implemented in ADAPT II. The parameters describing topotecan disposition between the plasma and CSF or ECF $\left(\mathrm{CL}_{\mathrm{in}}, \mathrm{CL}_{\text {out }}\right.$ and $\mathrm{V}_{\mathrm{ECF}}$ or $\mathrm{V}_{\mathrm{CSF}}$ ) were estimated for each individual mouse. The estimation was performed by fitting the model to the CSF or ECF data with the plasma pharmacokinetic parameters $\left(\mathrm{V}_{\mathrm{c}}, \mathrm{CL}_{\mathrm{t}}, \mathrm{V}_{\mathrm{p}}\right.$, and $\mathrm{CL}_{\mathrm{p}}$ ) fixed at the values obtained in the above described plasma pharmacokinetics section. To compare the noncompartmental and compartmental methods in estimation of $\mathrm{AUC}$ in the brain, we calculated the ratio of $\mathrm{CL}_{\text {in }}$ to $\mathrm{CL}_{\text {out }}$ obtained from the three-compartment analysis. Based on the relationship $\mathrm{CL}_{\text {in }} / \mathrm{CL}_{\text {out }}=\mathrm{AUC}_{\text {brain }} / \mathrm{AUC}_{\text {plasma }}$ (Wong et al., 1993), brain to plasma AUC ratios calculated from the noncompartmental and compartmental analyses were compared.

\subsubsection{Three-compartment analysis of plasma topotecan lactone and carboxylate data}

To calculate the plasma carboxylate AUC (where data were available), models were developed to fit plasma lactone and carboxylate data simultaneously. A three compartment model was best fitted to our dataset (two compartments for lactone and one compartment for carboxylate). The goodness of fit was based on comparing objective functions and diagnostic scatterplots. Prior to pharmacokinetic analysis for an individual mouse, a population analysis was conducted in NONMEM VI (subroutine ADVAN6) to obtain the population means and variations of the model parameters. The individual mouse pharmacokinetic parameters were then determined in ADAPT II by using maximum a posteriori probability (MAP) Bayesian estimation. The carboxylate AUC was simulated with the estimated parameters in ADAPT II. The plasma carboxylate AUC was used to calculate plasma lactone to carboxylate ratio of unbound topotecan. 


\subsubsection{Immunohistochemistry}

Brains were collected from FVB, Bcrpl $1^{(-/)}$, and $M d r l a / b^{(-/)}$mice (Taconic, Germantown, NY), fixed in 10\% neutral buffered formalin overnight, and embedded in paraffin before sectioning. Using immunohistochemistry procedures previously reported, the presence of Bcrp1 and P-gp was assessed in these tissues (Zhuang et al., 2006). The monoclonal antibody rat BXP-53 (1 $\mu \mathrm{g} / \mathrm{mL}$; Alexis Biochemicals, San Diego, CA) was used to detect Bcrp1 with rat IgG2a isotype as the negative control. The polyclonal rabbit anti-MDR antibody ( $2 \mu \mathrm{g} / \mathrm{mL}$; C-19, Santa Cruz Biotechnology, Inc., Santa Cruz, CA) and non-immunized rabbit IgG were used for P-gp detection in mouse brain and the negative control, respectively. Paraffin sections (3-5 $\mu \mathrm{m})$ were stained using standard avidin-biotin complex immunohistochemical techniques. This consisted of streptavidinhorseradish peroxidase combined with an antigen retrieval step (DAKO Corp., Carpinteria, CA), and the addition of an avidin-biotin (Vector Labs, Burlingame, CA) blocking step before primary antibody application. Primary antibodies were incubated overnight at $4^{\circ} \mathrm{C}$ followed by secondary antibody $(4 \mu \mathrm{g} / \mathrm{ml})$, either biotinylated anti-rat or anti-rabbit. Results were visualized with diaminobenzidine (DAKO), counterstained with haematoxylin (DAKO), and mounted in permanent medium (Fisher Scientific, Suwannee, GA).

\subsubsection{In vitro study of effect of $\mathrm{pH}$ on topotecan lactone and carboxylate transport by BCRP}

Saos2-BCRP or Saos2-pcDNA cells $\left(1 \times 10^{6}\right)$ were maintained in regular DMEM media $\left(10 \% \mathrm{FBS}, 1 \% \mathrm{~L}\right.$-glutamine) in $10 \mathrm{~cm}^{2}$ dishes for 16 hours at $37^{\circ} \mathrm{C}$. The media was carefully aspirated and the cells were washed twice with PBS (pH 6 or 8). For topotecan treatment at $\mathrm{pH} 6,4.5 \mu \mathrm{l}$ of $1 \mathrm{mg} / \mathrm{ml}$ topotecan in DMSO was diluted in $9 \mathrm{ml}$ $\mathrm{pH} 6 \mathrm{PBS}$ (at $37^{\circ} \mathrm{C}$ ). For topotecan treatment at $\mathrm{pH} 8,45 \mu \mathrm{l}$ of $0.1 \mathrm{mg} / \mathrm{ml}$ topotecan in $0.4 \mathrm{~N}$ Borax solution was diluted in $9 \mathrm{ml} \mathrm{pH} 8 \mathrm{PBS}$ (at $37^{\circ} \mathrm{C}$ ). The prepared topotecan treatment solution $(8 \mathrm{ml})$ was added to each $10 \mathrm{~cm}^{2}$ dish. The cells were incubated with topotecan solution for 1 minute or 30 minutes at $37^{\circ} \mathrm{C}$ without $\mathrm{CO}_{2}$. Treatment media $(200 \mu \mathrm{l})$ was removed and placed into $800 \mu \mathrm{l}$ cold methanol for topotecan extraction to determine the drug concentration left in the media. The plate was washed once with $5 \mathrm{ml}$ cold PBS at the appropriate $\mathrm{pH}(6$ or 8$)$. PBS $(1 \mathrm{ml})$ at either $\mathrm{pH} 6$ or 8 was added into the dishes and the cells were detached using a cell scraper. The cells were then lysed by sonication for 10 seconds 3 times. The lysate $(200 \mu \mathrm{l})$ was placed into $800 \mu \mathrm{l}$ cold methanol for topotecan extraction to determine the intracellular drug concentration. The remainder of the lysate was used for protein quantification by using Bradford protein assay (Bio-rad \#500-0006).

\subsubsection{Statistical analysis}

Statistical analysis was performed in Sigma Stat 3.1. Data were presented as mean \pm SD. For protein binding study, one-way ANOVA was performed. For vCSF study, 
one way ANOVA was performed to determine the significance of between group differences, followed by bonferroni-corrected multiple comparisons of wild type with each knockout model. For ECF study, Kruskal-Wallis one-way ANOVA was performed (data doesn't pass the normality test), followed by rank test to compare wild type and each knockout model. For gefitinib treatment study, student's $t$ test was performed to compare wild type and $M d r l a / b^{(-)} B c r p 1^{(-)}$. For lactone to carboxylate ratio study, oneway ANOVA was performed to determine the significance of vCSF between group differences, followed by Bonferroni-corrected multiple comparisons of wild type with each knockout model. Student's t test was performed to compare plasma and vCSF lactone to carboxylate AUC ratio within wild type or Mdrla/b $b^{(-)} B c r p 1^{(--)}$. For $\mathrm{pH}$ effect study, student's t test was performed to compare Saos2-BCRP and Saos2-pcDNA intracellular topotecan concentrations.

\subsection{Results}

\subsubsection{Topotecan lactone protein binding}

Topotecan lactone protein binding was assessed in each of the mouse models used in this study, including FVB, Mdrla $/ b^{(-)}, B c r p 1^{(-)}$, and $M d r 1 a / b^{(-)} / B c r p 1^{(--)}$. The topotecan lactone unbound fractions were determined to be $30.1 \pm 1.0 \%, 29.2 \pm 2.7 \%$, $30.9 \pm 2.2 \%$, and $27.5 \pm 1.9 \%$, respectively. One-way ANOVA analysis showed no significant difference among these results $(\mathrm{p}=0.10)$.

\subsubsection{Bcrp1 and P-gp enhance topotecan penetration into ventricular CSF}

To investigate the relative contribution of Bcrp1 and P-gp on topotecan penetration into ventricular CSF (i.e., through blood CSF barrier), FVB wild type and three targeted mutation mouse models $\left(M d r 1 a / b^{(-)}, B c r p 1^{(-)}\right.$, and Mdrla/b $b^{(-)} B c r p 1^{(-)}$) were used. A microdialysis probe was placed in the lateral ventricle of the mouse model as described above, and the probe location was verified with postmortem histology examination as depicted in (Figure 3-1A). After a single $4 \mathrm{mg} / \mathrm{kg}$ topotecan i.v. bolus injection, the $\mathrm{VCSF}$ to plasma ratio of unbound topotecan lactone was calculated for each animal. The $\mathrm{vCSF}$ to plasma $\mathrm{AUC}$ ratios $\left(\mathrm{AUC}_{\mathrm{u}, \mathrm{vCSF}} / \mathrm{AUC}_{\mathrm{u}, \mathrm{plasma}}\right)$ were determined as $2.85 \pm 0.14,2.30 \pm 0.12,1.50 \pm 0.05,0.85 \pm 0.05$ for FVB, Mdrla $/ b^{(-)}, B c r p 1^{(-)}$, and $M d r l a / b^{(--)} / B$ crpl $^{(--)}$, respectively (Figure 3-2). The in vivo probe recoveries were $8.1 \pm 2.4 \%, 7.1 \pm 1.1 \%, 7.5 \pm 1.5 \%$ and $6.0 \pm 0.6 \%$, respectively. The $\mathrm{CL}_{\text {in }} / \mathrm{CL}_{\text {out }}$ at the blood CSF barrier, determined by compartmental analysis, agreed well with the AUC ratio determined by noncompartmental analysis (Table 3-1). 

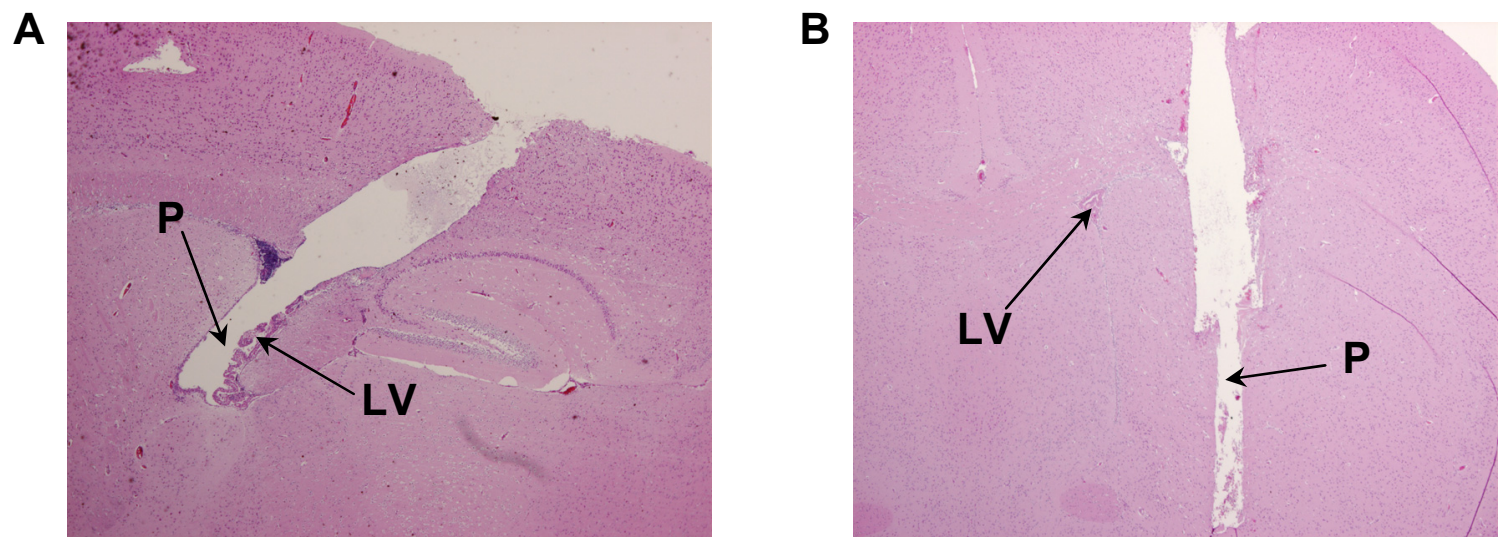

Figure 3-1 Microdialysis probe track in lateral ventricle or parenchymal tissue.

(A). Saggital section of probe track (P) insertion into the lateral ventricle (LV). (B). Coronal section of probe track insertion into the brain parenchyma. 

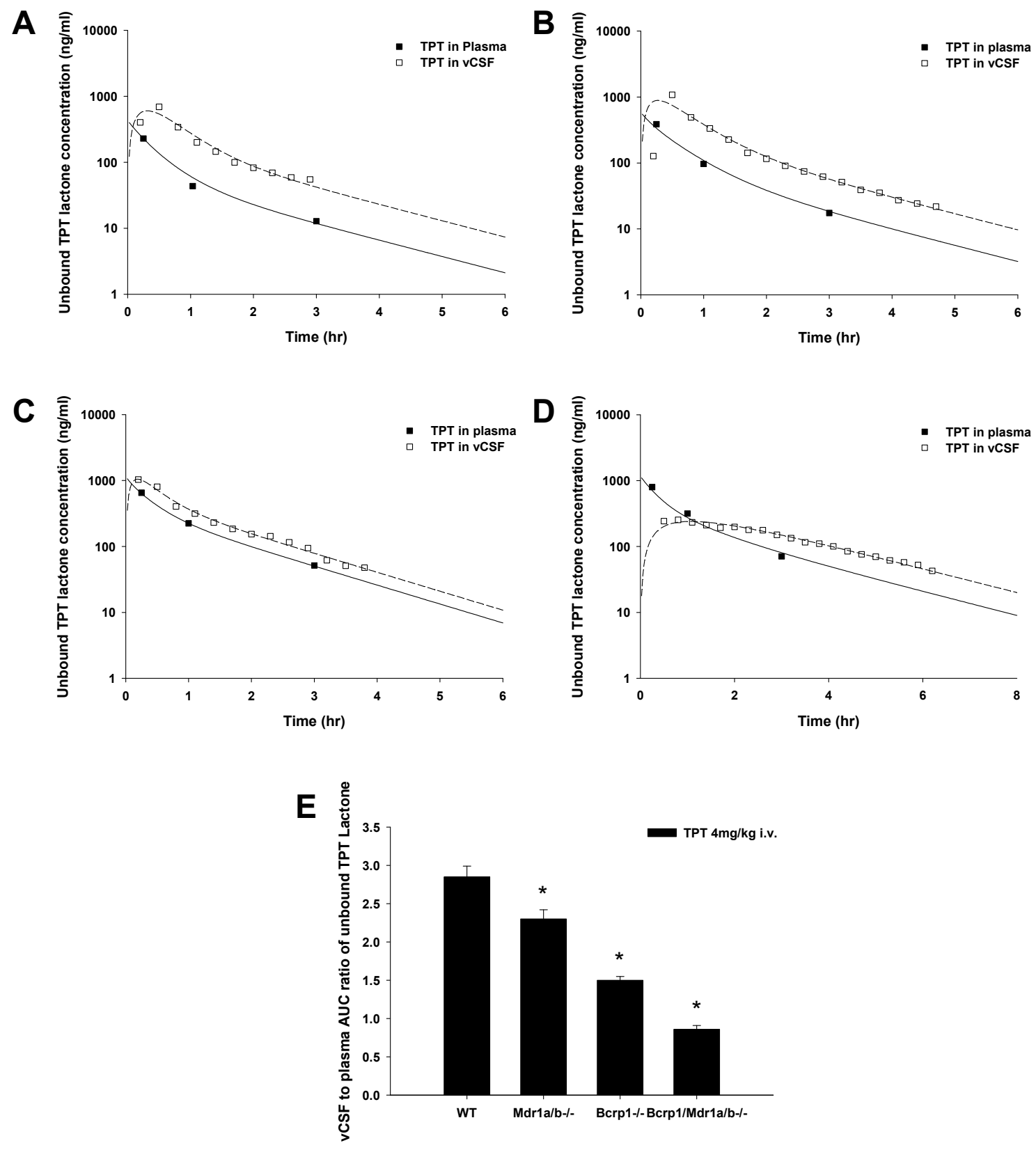

Figure 3-2 Topotecan lactone penetration into VCSF in various mouse models at one dose of topotecan $4 \mathrm{mg} / \mathrm{kg}$ i.v. bolus.

Individual representative unbound topotecan lactone concentration-time plots in vCSF

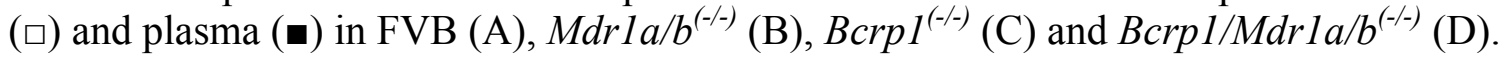
(E). vCSF to plasma AUC ratio of unbound topotecan lactone in various models. (One way ANOVA, $\mathrm{F}=351, \mathrm{P}<0.05$; Bonferroni-corrected multiple comparison between WT and each knockout model, $* \mathrm{P}<0.05$ ) 
Table 3-1 Noncompartmental (AUC ratio) and compartmental analysis (CLin/CLout) for brain (CSF or ECF) to plasma AUC ratio of unbound topotecan lactone in various mouse models.

\begin{tabular}{|c|c|c|c|c|}
\hline \multirow[b]{2}{*}{ Mouse model } & \multicolumn{2}{|c|}{ vCSF } & \multicolumn{2}{|c|}{ ECF } \\
\hline & AUC ratio & CLin/CLout & AUC ratio & CLin/CLout \\
\hline \multicolumn{5}{|l|}{ Topotecan $4 \mathrm{mg} / \mathrm{kg}$} \\
\hline WT & $2.85 \pm 0.14(\mathrm{n}=6)$ & $3.01 \pm 0.15$ & $0.35 \pm 0.04(\mathrm{n}=5)$ & $0.36 \pm 0.06$ \\
\hline$M d r 1 a / b^{(--)}$ & $2.30 \pm 0.12(\mathrm{n}=4)$ & $2.57 \pm 0.17$ & N/A & N/A \\
\hline$B \operatorname{crp} 1^{(--)}$ & $1.50 \pm 0.05(\mathrm{n}=3)$ & $1.59 \pm 0.16$ & $0.35 \pm 0.01(\mathrm{n}=3)$ & $0.42 \pm 0.06$ \\
\hline Bcrpl/Mdrla/b/(--) & $0.86 \pm 0.05(\mathrm{n}=5)$ & $0.86 \pm 0.04$ & $0.81 \pm 0.04(\mathrm{n}=3)$ & $0.89 \pm 0.07$ \\
\hline \multicolumn{5}{|l|}{ Topotecan $4 \mathrm{mg} / \mathrm{kg}+$} \\
\hline \multicolumn{5}{|l|}{ Gefitinib $200 \mathrm{mg} / \mathrm{kg}$} \\
\hline WT & $1.15 \pm 0.16(\mathrm{n}=3)$ & $1.25 \pm 0.14$ & $0.63 \pm 0.03(n=3)$ & $0.74 \pm 0.14$ \\
\hline Bcrpl/Mdrla/b(--) & $1.10 \pm 0.14(\mathrm{n}=3)$ & $1.13 \pm 0.13$ & $0.95 \pm 0.02(\mathrm{n}=3)$ & $1.08 \pm 0.03$ \\
\hline
\end{tabular}




\subsubsection{Bcrp1 and P-gp decrease topotecan penetration into brain ECF}

Using a microdialysis sampling technique, the relative contribution of Bcrp1 and P-gp on topotecan penetration into brain ECF was assessed in transporter deficient mouse models. As with the ventricular CSF studies, the location of the probe was verified with postmortem histology examination as depicted in Figure 3-1B. The ECF to plasma AUC ratios $\left(\mathrm{AUC}_{\mathrm{u}, \mathrm{ECF}} / \mathrm{AUC}_{\mathrm{u} \text {,plasma }}\right)$ of unbound topotecan lactone were determined as $0.35 \pm 0.04,0.35 \pm 0.01$, and $0.81 \pm 0.04$ for FVB, Bcrpl ${ }^{(--)}$, and $M d r l a / b^{(--)} B_{c r p l} I^{(--)}$, respectively (Figure 3-3). The in vivo probe recoveries were $8.5 \pm 2.4 \%, 5.4 \pm 0.6 \%$, and $4.3 \pm 0.2 \%$, respectively. The topotecan lactone ECF concentrations in the $M d r 1 a / b^{(-)}$ were at the lower limit of quantitation of the assay, so it was not possible to report an ECF to plasma AUC ratio (Leggas et al., 2004c; Zhuang et al., 2006). The $\mathrm{CL}_{\text {in }} / \mathrm{CL}_{\text {out }}$ across the blood brain barrier determined by compartmental analysis agreed with the AUC ratio as estimated by the noncompartmental analysis (Table 3-1).

\subsubsection{Differential regulatory effects of gefitinib on topotecan penetration into vCSF and ECF}

To modulate the effect of active transporters on topotecan CNS penetration, the tyrosine kinase inhibitor gefitinib was administered to FVB and $M d r l a / b^{(-)} B c r p 1^{(-/)}$ mice one hour prior to the topotecan dose. After gefitinib pretreatment, similar topotecan lactone vCSF penetration (i.e., $\mathrm{AUC}_{\mathrm{u}, \mathrm{vCSF}} / \mathrm{AUC}_{\mathrm{u}, \text { plasma }}$ ) was determined in $\mathrm{FVB}$ $(1.15 \pm 0.16)$ and $M d r l a / b^{(--)} B c r p 1^{(--)}$mice $(1.1 \pm 0.14)$ (see Figure 3-4A, 3-4B), These values were not significantly different (Student's t test, $p=0.27$ ). These results suggest gefitinib fully inhibits Bcrp1 and P-gp function at the blood CSF barrier.

These results were compared with the effect of gefitinib on topotecan lactone ECF penetration. In FVB mice, gefitinib pretreatment led to a topotecan lactone ECF penetration (e.g., $\mathrm{AUC}_{\mathrm{u}, \mathrm{ECF}} / \mathrm{AUC}_{\mathrm{u} \text {,plasma) }}$ of $0.63 \pm 0.03$ (Figure 3-4C), which increased to $0.95 \pm 0.02$ in $M d r l a / b^{(--)} B c r p 1^{(-/)}$mice (Student's t test, $\mathrm{p}<0.05$ ) (Figure 3-4D). Thus, gefitinib may exert effects on Bcrp1 and P-gp function to different degrees at the blood brain barrier than at the blood CSF barrier.

\subsubsection{Preferential disposition of topotecan lactone in vCSF}

As Bcrp1 was identified as the primary transport protein responsible for topotecan penetration into ventricular CSF, the preference for unbound topotecan lactone and carboxylate was further studied. First, the vCSF carboxylate AUC value was calculated for FVB and transporter deficient mouse models. The vCSF lactone to carboxylate AUC ratio $\left(\mathrm{AUC}_{\text {lactone, } \mathrm{vCSF}} / \mathrm{AUC}_{\text {carboxylate, } \mathrm{vCSF}}\right)$ was determined as $5.7 \pm 0.8,3.9 \pm 0.6,3.6 \pm 0.5$, and $0.8 \pm 0.2$ in FVB, Mdrla $/ b^{(--)}, B c r p 1^{(-)}$, and $M d r l a / b^{(-)} B c r p 1^{(--)}$, respectively (Figure 35). Correspondingly the plasma lactone to carboxylate AUC ratio was $1.24 \pm 0.38$ in FVB and $0.95 \pm 0.28$ in $M d r l a / b^{(-/)} B_{c r p} 1^{(-/)}$. These results suggested the higher lactone penetration into the vCSF cannot be attributed to the plasma lactone level 

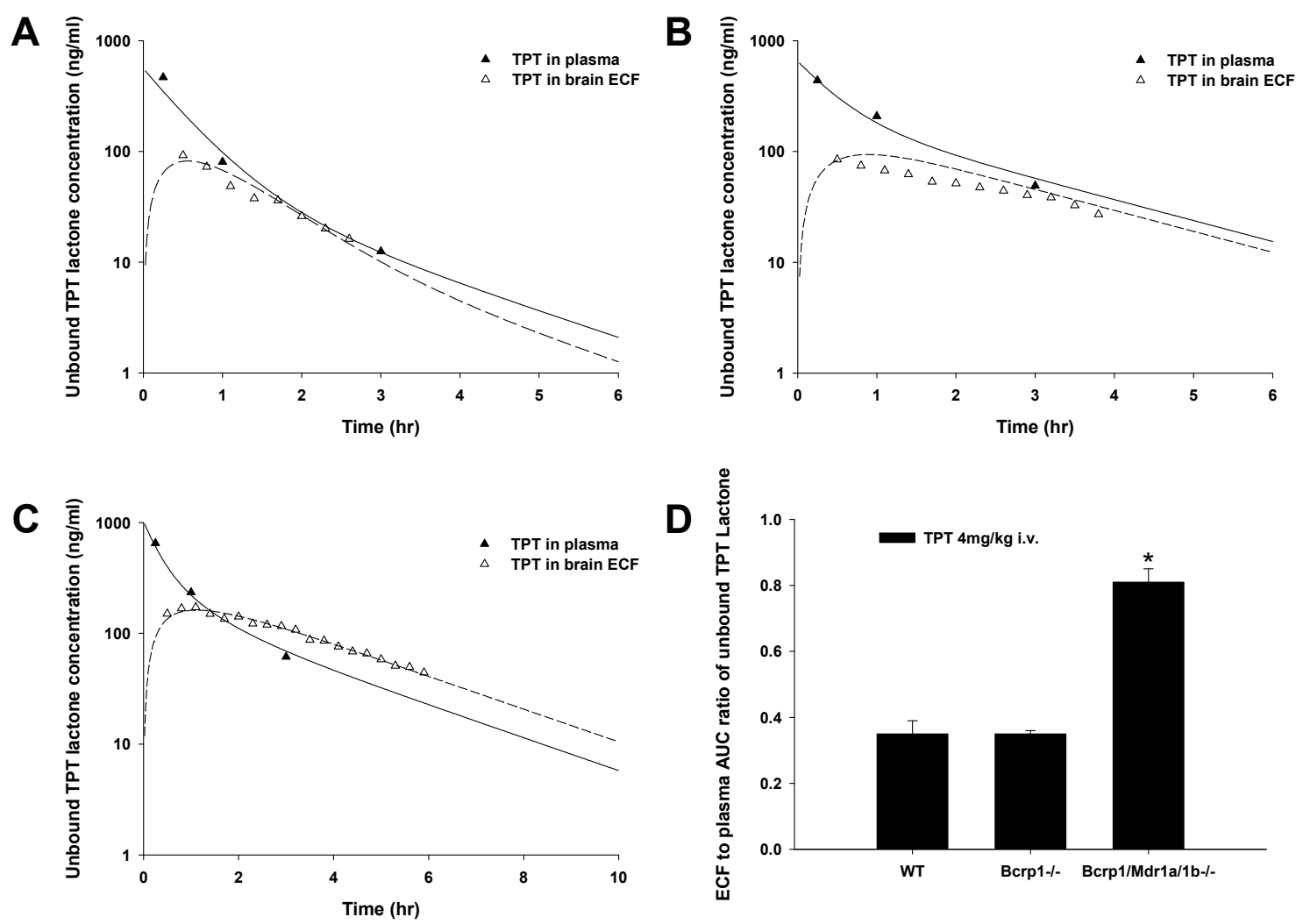

Figure 3-3 Topotecan lactone penetration into brain ECF in various mouse models at one dose of topotecan $4 \mathrm{mg} / \mathrm{kg}$ i.v. bolus.

Individual representative unbound topotecan lactone concentration-time plots in ECF $(\Delta)$ and plasma ( $\mathbf{\Delta})$ in FVB (A), Bcrpl ${ }^{(--)}(\mathrm{B})$ and Bcrpl/Mdrla/b ${ }^{(-)}(\mathrm{C})$. D, brain ECF to plasma AUC ratio of unbound topotecan lactone in various models. (Kruskal-Wallis one way ANOVA, $\mathrm{P}<0.05$; Each knockout model was compared with $\mathrm{WT},{ }^{*} \mathrm{P}<0.05$ ) 

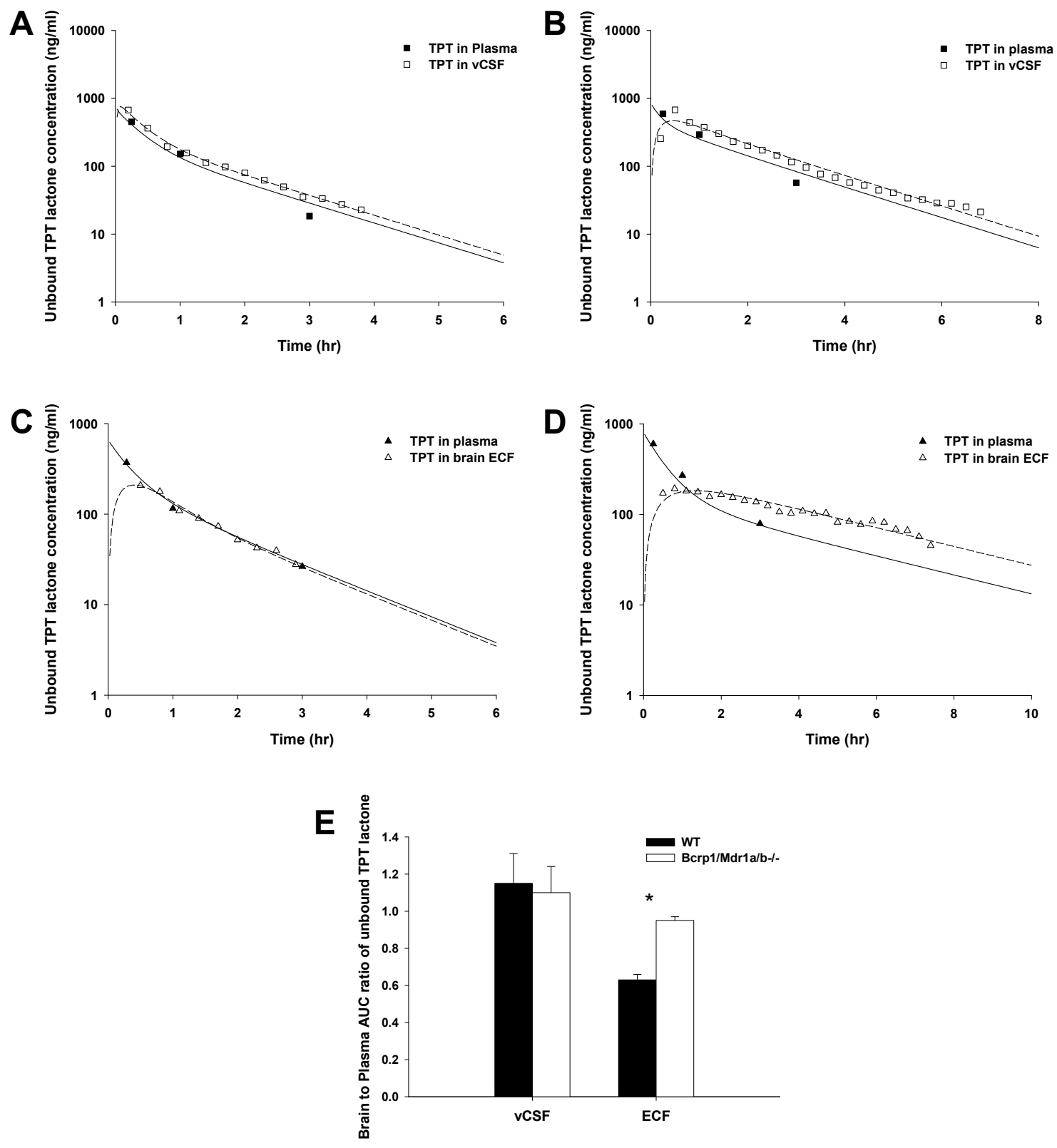

Figure 3-4 Regulatory effect of gefitinib on topotecan penetration into brain ECF or vCSF at one dose of topotecan $4 \mathrm{mg} / \mathrm{kg}$ i.v. bolus and gefitinib $200 \mathrm{mg} / \mathrm{kg}$ oral gavage pretreatment.

Individual representative unbound topotecan lactone concentration-time plots in vCSF $(\mathrm{A}, \mathrm{B} ; \square), \mathrm{ECF}(\mathrm{C}, \mathrm{D} ; \Delta)$ and plasma $(\boldsymbol{\square}, \mathbf{\Delta})$ with oral gefitinib pretreatment in FVB (A, C) and $B c r p 1 / M d r l a / b^{(--)}(\mathrm{B}, \mathrm{D})$. E, Brain (vCSF or ECF) to plasma AUC ratio of unbound topotecan lactone in FVB and Bcrpl/Mdrla/b(-). (Student's t-test, WT compared with Bcrpl/Mdrla/b $\left.b^{(--)}, * \mathrm{P}<0.05\right)$ 


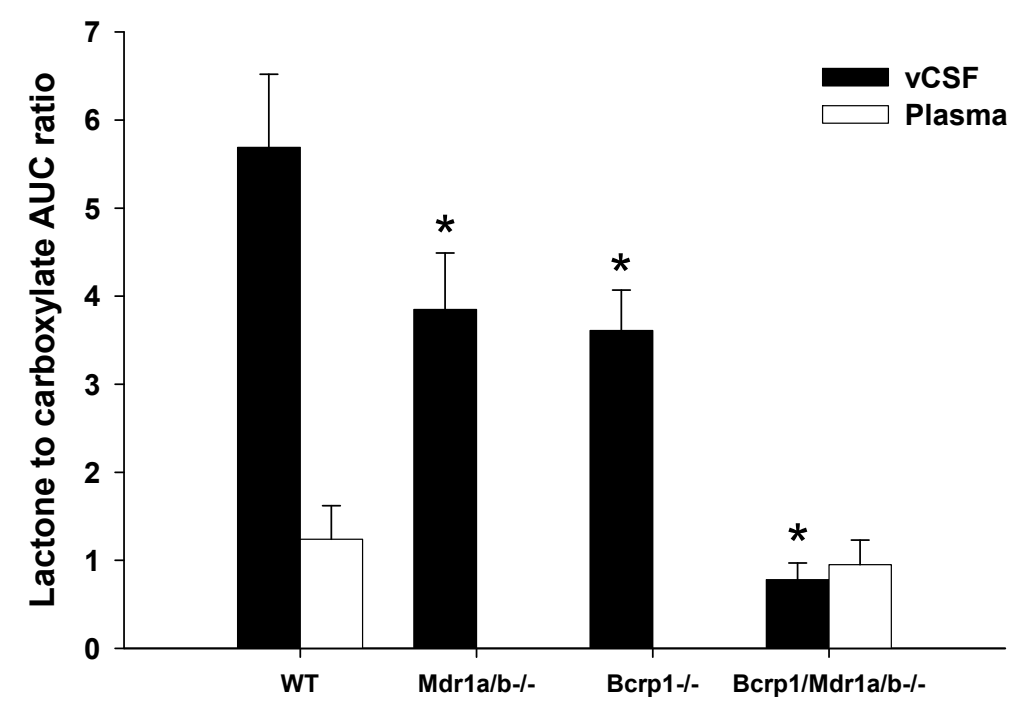

Figure 3-5 vCSF lactone to carboxylate AUC ratio of unbound topotecan.

vCSF (black column) lactone to carboxylate AUC ratio of unbound topotecan were determined in various mouse models and compared with the plasma lactone to carboxylate AUC ratio of unbound topotecan. (For plasma group, Bcrpl/Mdrla/ $b^{(--)}$was compared with WT, student's t-test. For vCSF group, one way ANOVA, F=51.8, $\mathrm{P}<0.05$; Bonferroni-corrected multiple comparison between each knockout model and WT, $* \mathrm{P}<0.05)$ 
but rather to a consequence of active transport across the $\mathrm{BCB}$. This would also suggest that Bcrp1 and P-gp transport the lactone form more effectively than the carboxylate form.

\subsubsection{Saos2-BCRP cells preferentially transport topotecan lactone}

To assess if BCRP will preferentially transport topotecan lactone or carboxylate, Saos2-BCRP cells were incubated with topotecan at different $\mathrm{pH}$ values to shift the distribution of lactone and carboxylate forms. At $\mathrm{pH} 6$ after 1 min incubation of topotecan lactone, the intracellular topotecan lactone concentration was determined as $0.063 \pm 0.01 \mathrm{ng} / \mu \mathrm{g}$ protein in Saos2-pcDNA and $0.043 \pm 0.005 \mathrm{ng} / \mu \mathrm{g}$ protein in Saos2BCRP $(p<0.05$, Student's t-test). Similarly after $30 \mathrm{~min}$ incubation the intracellular topotecan lactone concentration was determined as $0.039 \pm 0.003 \mathrm{ng} / \mu \mathrm{g}$ protein in Saos2pcDNA and $0.026 \pm 0.0043 \mathrm{ng} / \mu \mathrm{g}$ protein in Saos2-BCRP ( $<<0.05$, Student's t-test). Topotecan carboxylate was not detected at pH 6 (Figure 3-6A, 3-6B). At pH 8 after 1 min incubation of topotecan carboxylate, the intracellular topotecan carboxylate concentration was determined as $0.03 \pm 0.003 \mathrm{ng} / \mu \mathrm{g}$ protein in Saos2-pcDNA and $0.028 \pm 0.04 \mathrm{ng} / \mu \mathrm{g}$ protein in Saos2-BCRP ( $\mathrm{p}=0.6$, Student's t test). Similarly after $30 \mathrm{~min}$ incubation the intracellular topotecan carboxylate concentration was determined as $0.02 \pm 0.007 \mathrm{ng} / \mu \mathrm{g}$ protein in Saos2-pcDNA and $0.02 \pm 0.002 \mathrm{ng} / \mu \mathrm{g}$ protein in Saos2-BCRP ( $p=0.9$ Student's $t$ test). Topotecan lactone was not detected at $\mathrm{pH} 8$ (Figure 3-6C, 3-6D). These results demonstrated that BCRP preferentially transports the lactone form but not the carboxylate form of topotecan in vitro.

\subsection{Discussion}

The active transport mechanisms that control topotecan CNS penetration and pharmacokinetics in vivo are not clearly described. In this study, by using ABC transporters-targeted mutation mouse models, microdialysis sampling technique in discrete brain compartments and pharmacokinetic modeling, we demonstrated the differential action of Bcrp1 and P-gp in topotecan brain penetration through the CNS barriers: on one hand, they enhance topotecan accumulation into the CSF through their effect on the $\mathrm{BCB}$; on the other, they restrict parenchymal brain topotecan exposure by their role as efflux transporters in the BBB.

P-gp and Bcrp1 have been recently reported by Tellingen et al to work together in constraining topotecan brain penetration (de Vries et al., 2007). In their study., whole brain tissue homogenates of knockout mice $\left(M d r l a / b^{(-/)}, B \operatorname{crpl} 1^{(-/)}, M d r 1 a / b^{(-/)} B \operatorname{crp} 1^{(-/)}\right.$,

and $\mathrm{Mrp}^{(--)}$) receiving topotecan were analyzed, showing that both P-gp and BCRP, but not MRP4, limit topotecan concentration in brain homogenates (de Vries et al., 2007). Although they obtained significant differences in topotecan penetration between animal groups depending on the presence or absence of $\mathrm{ABC}$ transporters and the inhibitor elacridar, the sampling technique they used is, by itself, a potential source of misleading results. In fact, their samples represent a mixture of three compartments found in the 

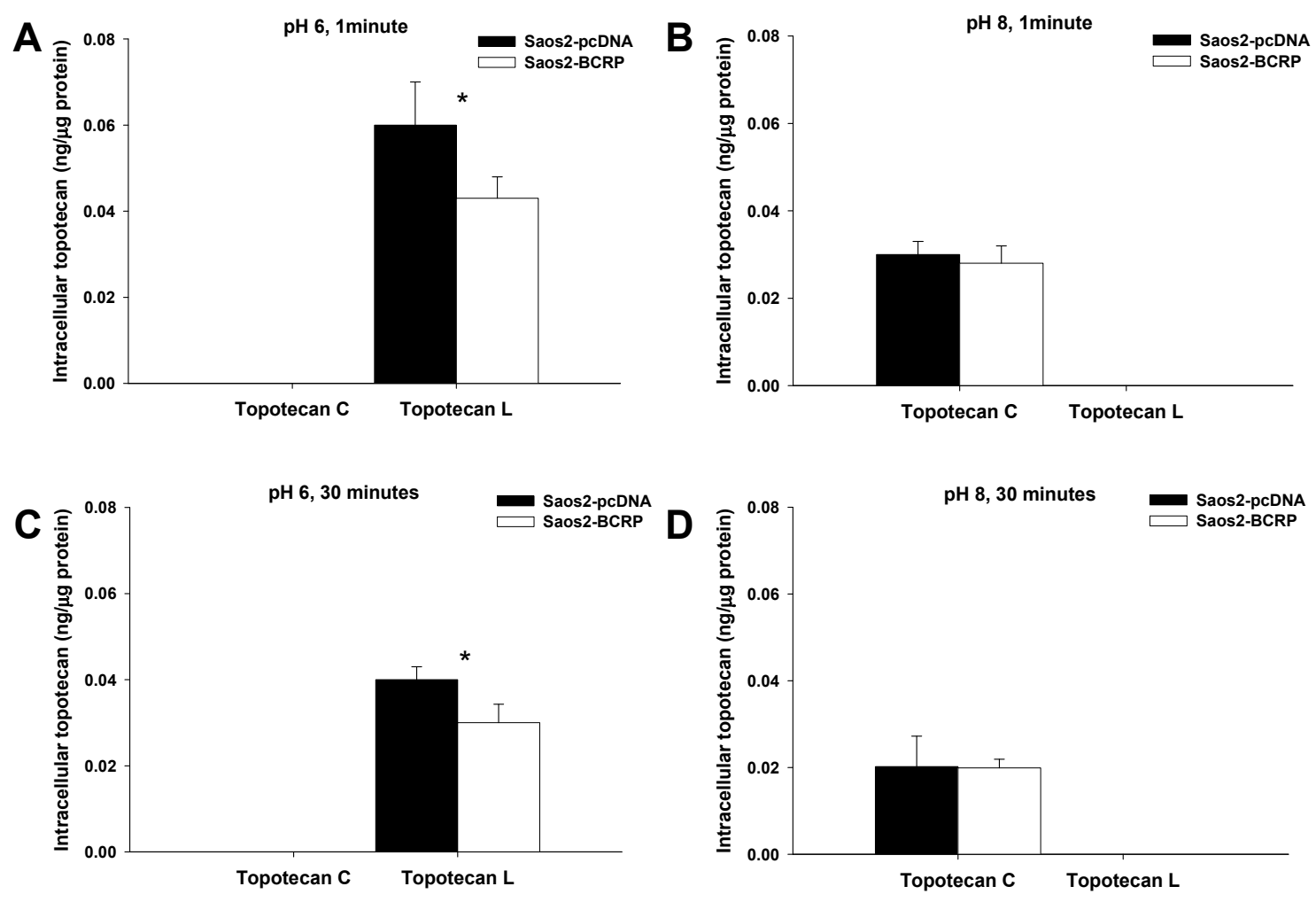

Figure 3-6 pH effect on topotecan lactone and carboxylate transport by BCRP.

Saos2-pcDNA (black column) and Saos2-BCRP (white column) cells were incubated with topotecan solution prepared in either $\mathrm{pH} 6$ (A and $\mathrm{C}$ ) or $\mathrm{pH} 8$ (B and D) for 1 minute (A and $\mathrm{B}$ ) or 30 minutes (C and D). After incubation the medium was removed and the intracellular concentration of topotecan lactone or carboxylate was determined. (Student's t-test, $* \mathrm{P}<0.05$ ). 
brain, the CSF compartment (regulated by the $\mathrm{BCB}$ ), the ECF compartment of brain parenchyma (regulated by the $\mathrm{BBB}$ ), and the vascular compartment (separated from the other two compartments by both the $\mathrm{BBB}$ and the $\mathrm{BCB}$ ). We and others have recently reported on the differential accumulation of drugs in these three compartments (StainTexier et al., 1999; Zhuang et al., 2006). Remarkably, Stain-Texier et al. found sharpdifferences among the results obtained by analyzing morphine-6-glucoronide in brain ECF (sampled using microdialysis), CSF (sampled by puncture aspiration) or whole brain tissue (homogenates), determining a high accumulation of this morphine metabolite in brain ECF that could not have been determined by the brain homogenate technique. Similarly, we recently reported a differential accumulation of topotecan in cerebral compartments of FVB mice treated with gefitinib: increased concentrations in ECF and decreased in vCSF (Zhuang et al., 2006).

The homogenate method used by Tellingen et al was unable to assess another two key parameters in topotecan pharmacokinetics: 1) bound and unbound drug fractions, and 2 ) topotecan lactone-to-carboxylate ratio. The last question is especially relevant since even though simple HPLC methods are available to analyze topotecan and carboxylate forms simultaneously, in the mentioned study the samples were acidified to transform topotecan in the lactone form (de Vries et al., 2007). In contrast, by using the microdialysis technique we were able to accomplish with the challenging task of analyzing simultaneously both topotecan carboxylate and lactone unbound concentrations in the brain parenchymal ECF and the ventricular CSF. The technical difficulty of placing the probe precisely into the mouse lateral ventricle for vCSF sampling was overcome by a modified surgical procedure that takes advantage of the conventional features of a stereotaxic instrument and the tridimensional interpretation of the previously established knowledge on the mouse brain anatomy (Shen et al., 2008). The procedure allowed a continuous sampling in awake animals, defining individual pharmacokinetic profiles and reducing the number of laboratory animals employed in the study.

After evaluating the plasma protein binding of lactone topotecan in our mouse models, vCSF to plasma AUC ratios of unbound topotecan lactone could be determined, serving as an invaluable indicator for drug penetration through BCB (HammarlundUdenaes et al., 1997; Wong et al., 1993). By comparing the AUC ratios of the knockout mouse models to that of FVB wild type, the relative role of Bcrp1 and P-gp in topotecan vCSF penetration could be clearly defined. The results suggest that Bcrp1 plays a major role (AUC ratios were 1.5 vs 2.85 in $B c r p 1^{(--)}$and FVB mice, respectively) and P-gp plays a relatively minor role (AUC ratios were 2.3 vs 2.85 in $M d r l a / b^{(--)}$and FVB mice, respectively) in enhancing topotecan penetration in the $\mathrm{vCSF}$. The results also indicate there was no apparent functional redundancy between Bcrp1 and P-gp at BCB as deficiency of each protein caused corresponding reduced topotecan penetration. Furthermore, the relatively low AUC ratio (0.85) found in the Bcrpl/Mdrla $/ b^{(--)}$model suggests that other efflux mechanisms may be involved. This is in agreement with our previous finding that MRP4 restricts topotecan vCSF penetration (Leggas et al., 2004a). In Bcrp $1 / M d r l a / b^{(-)}$model the unbound topotecan concentrations in vCSF were maintained higher than in plasma in later drug elimination phase (Figure 3-2D). This 
suggests in addition to Bcrp1 and P-gp there exist other mechanisms taking up drug from blood to vCSF.

In contrast, the role of Bcrp1 or P-gp in BBB penetration cannot be defined separately as well as in BCB. In the $M d r l a / b^{(--)}$model Bcrp1 mRNA of cerebral microvessels was reported to be upregulated by 3 times (Cisternino et al., 2004). This is also substantiated by the stronger Bcrp1 immunohistochemical staining in $M d r 1 a / b^{(--)}$ than the FVB wild type mice (Figure 3-7). Although Tellingen et al. reported the Bcrp1 protein level was not changed in this model by using western blot from brain homogenate, it is highly probable the protein expression alteration at microvessels was masked by brain tissues (de Vries et al., 2007). Thus the $M d r l a / b^{(-/)}$model cannot be used reliably to verify the P-gp role in vivo in current experimental settings. In the $B$ crp $^{(--)}$model, although there has been no report of upregulation of any compensatory mechanism, the ECF to plasma AUC ratio was determined as $0.35 \pm 0.01$, which was comparable with FVB $(0.35 \pm 0.04)$. This result agreed with what Tellingen et al. reported that no penetration difference has been found between Bcrpl $^{(--)}$and wild type (de Vries et al., 2007). However Bcrp1 and P-gp together play a major role in restriction of topotecan penetration through BBB. This was substantiated by the ECF to plasma AUC ratio of unbound topotecan lactone $(0.81 \pm 0.04)$ in the $B c r p 1 / M d r 1 a / b^{(-/)}$model. Similar to the situation in vCSF, besides Bcrp1 and P-gp, other mechanism(s) taking up drug from blood to ECF may be involved in the topotecan ECF penetration (Figure 3-3C).

With clear understanding of the mechanisms (e.g. Bcrp1 and P-gp) that regulate topotecan $\mathrm{CNS}$ penetration through $\mathrm{BCB}$ and $\mathrm{BBB}$ we further investigated how these mechanisms can be modulated by a tyrosine kinase inhibitor, gefitinib. FVB and Bcrp $1 / M d r l a / b^{(--)}$mouse models were used for the investigation. Our previous studies have demonstrated that gefitinib increased oral absorption and decreased systemic clearance of topotecan (Leggas et al., 2006; Stewart et al., 2004b). In the current study we are particularly interested in the role of gefitinib on modulating $\mathrm{BBB}$ and $\mathrm{BCB}$. The rationale is if gefitinib fully inhibited Bcrp1 and $\mathrm{P}$-gp function at $\mathrm{BBB}$ or BCB we would see the same AUC ratios in FVB and Bcrpl/Mdrla/b ${ }^{(-)}$mice. At BCB with oral gefitinib pretreatment, the $\mathrm{AUC}_{\mathrm{u}, \mathrm{vCSF}} / \mathrm{AUC}_{\mathrm{u} \text {,plasma }}$ was $1.15 \pm 0.16$ in $\mathrm{FVB}$ and $1.10 \pm 0.14$ (p value $=0.88)$ in $B c r p 1 / M d r l a / b^{(--)}$which suggested gefitinib fully inhibited Bcrp1 and Pgp function. However at $\mathrm{BBB} \mathrm{AUC}_{\mathrm{u}, \mathrm{ECF}} / \mathrm{AUC}_{\mathrm{u} \text {,plasma }}$ was determined as $0.63 \pm 0.03$ in FVB and $0.95 \pm 0.02 \mathrm{Bcrp} 1 / \mathrm{Mdrla} / \mathrm{b}^{(--))}$( $\mathrm{p}$ value $\left.<0.001\right)$, which suggested gefitinib cannot fully inhibit Bcrp1 and P-gp function at BBB (see Appendix for more strict reasoning).

As mentioned above, both vCSF and ECF studies in Bcrp $1 / M d r 1 a / b^{(-/)}$model (Figure 3-2D, 3-3C, 3-4B, 3-4D) have suggested there are other ATP dependent and independent mechanisms involved in topotecan transport across $\mathrm{BCB}$ and $\mathrm{BBB}$. Recently a SLC22 family protein OAT3 has been shown to play a role in the rat renal tubular secretion of topotecan (Matsumoto et al., 2007). This protein is known to be expressed on the apical side of choroid plexus at BCB and basolateral side of endothelial cells at BBB (Loscher and Potschka, 2005a; Nagata et al., 2002). In addition SLC21 family proteins (organic anion transporting polypeptides, OATPs) are also widely expressed on BBB and 


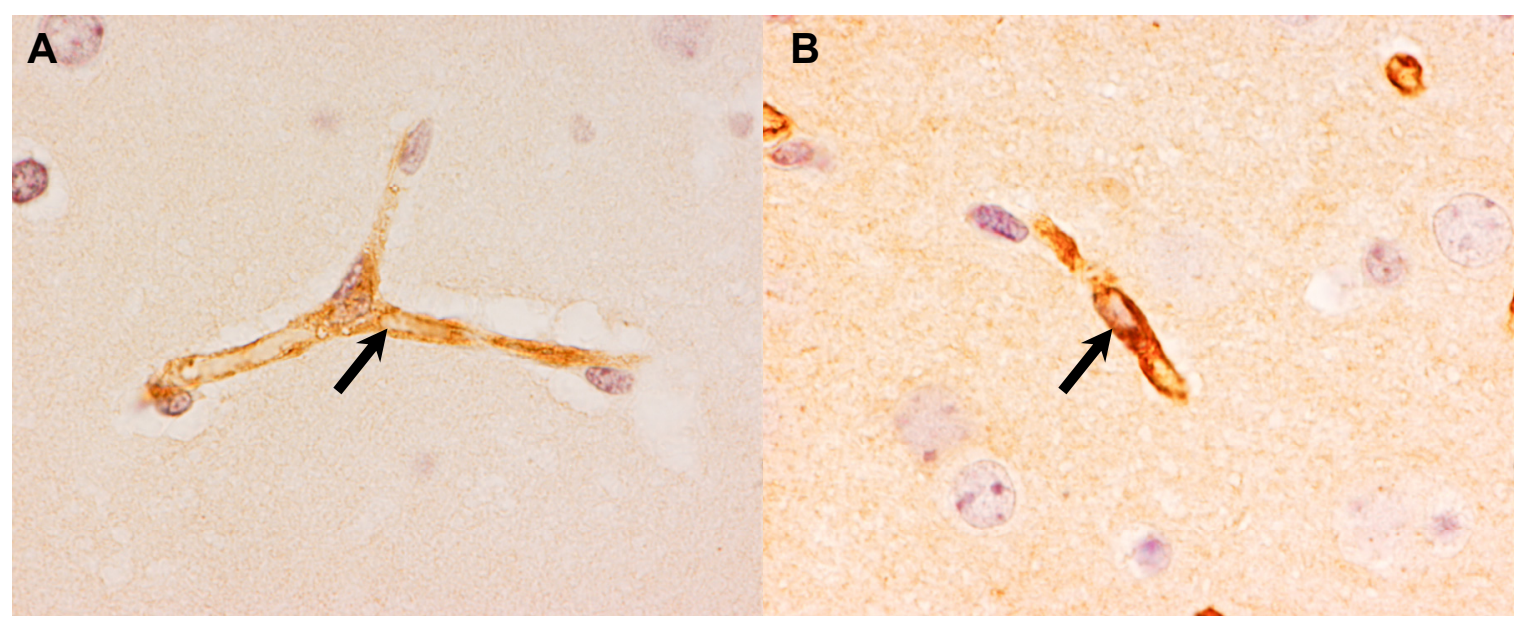

Figure 3-7 IHC staining of Bcrp in FVB and Mdr1a/b ${ }^{(--)}$.

Immunohistochemical staining of Bcrp1 at brain capillary vessels (arrows) in FVB wild type $100 \mathrm{X}(\mathrm{A})$ and $M d r 1 a / b^{(--)} 100 \mathrm{X}(\mathrm{B})$. The staining was stronger in Mdrla $/ \mathrm{b}^{(--)}$than in wild type. 
BCB. For examples, rat Oatp2 is expressed on both apical and basolateral sides of capillary endothelial cells and also expressed on the basolateral side of choroid plexus epithelial cells (Gao et al., 1999). Mouse Oatp3 is localized at the apical side of choroid plexus epithelial cells (Ohtsuki et al., 2004). Although these transporters are not reported to transport topotecan directly it is reported the human counterpart OATP-C can uptake another camptothecin analog, irinotecan and its active metabolite SN-38 (Nozawa et al., 2005). Taken together, these uptaking transporters can work with or against $A B C$ transporters, depending on their subcellular localizations, to regulate drug penetration into the CNS. For example, the basolateral localized Oatp2 at BCB can work with Bcrp1 and P-gp to enhance topotecan penetration into CSF; on the contrary apical localized OAT3 at BCB can work against Bcrp1 and P-gp but with MRP4 to restrict topotecan penetration. The effect of these uptaking mechanisms becomes significant especially when the major efflux transporters, Bcrp1 and P-gp, were absent (Figure 3-2D, 3-3C, 34B, 3-4C). Despite involvement of other transporters, the Bcrp1 and P-gp mediated mechanisms still dominated topotecan transport across BBB and BCB.

The clear understanding of transporting mechanisms for the drugs intended to treat brain tumors and other CNS diseases has significant clinical relevance (Kaddoumi et al., 2007). Only with such knowledge can an appropriate and effective modulation strategy be identified to avoid the drawback of drug penetration. Furthermore, brain penetration should also be considered even for the drugs not meant to treat brain tumors. This is largely because the drugs with poor brain penetration tend to make the brain a safe heaven for metastatic tumor cells. It is roughly estimated $10 \%-30 \%$ of cancer patients eventually developed brain metastasis (Palmieri et al., 2007; Patel and Mehta, 2007). Therefore, clearly defining the CNS penetration property of a new drug deserves more attention in the drug development process. 


\section{Chapter 4. Topotecan Penetration in an Intracranial Human Glioblastoma (U-87) Mouse Model}

\subsection{Introduction}

Central nervous system (CNS) tumors that cannot be managed by surgery and radiation will require adjuvant chemotherapy. However the role of chemotherapy in treatment of CNS tumors in a clinical setting is not clear. For example, camptothecin analogs (e.g. topotecan) are a promising class of agents that has demonstrated potent antitumor activity in preclinical CNS tumor xenograft studies (Hare et al., 1997; Houghton et al., 1995). However in clinical studies, only a modest response was observed in medulloblastoma patients, and very poor response was observed in malignant glioma patients (Nicholson et al., 2007; Stewart et al., 2004a; Chintagumpala et al., 2006). One probable reason is the presence of the blood brain barrier (BBB), which restricts the CNS penetration of the chemotherapeutic agents.

Drug CNS penetration is determined by multiple factors including drug physiochemical properties, drug protein binding fraction, and drug influx or efflux transporter systems. Our previous work demonstrates topotecan penetration into normal brain parenchymal tissue was restricted by the efflux $\mathrm{ABC}$ transporters (e.g. Bcrp1 and Pgp) located at BBB (Zhuang et al., 2006). The pathological conditions of the tumor itself add another layer of complexity to understanding topotecan penetration into CNS tumors.

The CNS tumor BBB is often referred to as blood brain tumor barrier (BTB). $\mathrm{BTB}$ has distinct properties from normal BBB. First, many tight junction proteins (e.g. ZO-1, occludin and claudin) are downregulated at BTB (Sawada et al., 2000; Liebner et al., 2000; Papadopoulos et al., 2001). Second, the vasculature in solid tumors usually appears in abnormal morphology, which results in a torturous and irregular vessel lumen (Baluk et al., 2005). Third, there exists active angiogenesis activity in tumor (Ferrara, 2004; Nabors et al., 2001; Strugar et al., 1995; Weindel et al., 1994). The effects of all these uncommon activities in tumor on drug delivery can become quite complicated and unpredictable. For example, the disregulated tight junction and active angiogenesis activities produce a more permeable blood vessel, which might increase drug delivery (Devineni et al., 1996a; Dukic et al., 2004). On the other hand, the abnormal blood vessel reduces blood flow, which could decrease drug delivery. Furthermore, a more permeable vasculature might cause increased interstitial fluid pressure (IFP), which resists drug delivery from blood to tissue. In a rat model, normalizing the tumor blood vessel increased topotecan delivery to neuroblastoma (Dickson et al., 2007). However the same strategy (normalizing the tumor blood vessel) does not seem to work for CNS tumor (Devineni et al., 1996b; Claes et al., 2008).

Although topotecan penetration studies have been done in other tumors (e.g. neuroblastoma) (Dickson et al., 2007), the data cannot be directly extrapolated for gliomas due to the distinct features of CNS tumors (e.g. blood brain barrier). A clearer understanding of drug disposition in the tumor itself is critical for developing effective 
therapy. If topotecan concentrations in tumor can be measured, a model can then be constructed to relate the drug tumor exposure to plasma exposure. Based on that model, a target exposure level can be reached by controlling the plasma exposure, which is clinically more convenient. For example, in a pediatric medulloblastoma trial, when topotecan lactone CSF concentrations were measured (medulloblastoma has extensive access to CSF), a three-compartment model was constructed to relate CSF concentrations to plasma concentrations. With the model, a CSF target exposure ( $>1 \mathrm{ng} / \mathrm{ml}$ for $8 \mathrm{hr}$ ) determined through preclinical study (Zamboni et al., 1998) can be reached by maintaining a plasma exposure between 120 and $160 \mathrm{ng} / \mathrm{ml} \cdot \mathrm{hr}$ (Stewart et al., 2004a). The clinical outcome was also encouraging because $28 \%$ of the patients responded to the therapy and $47 \%$ of the patients had their diseases stabilized (Stewart et al., 2004a). Currently however, reliable topotecan disposition data in gliomas is still lacking.

It would be of great clinical interest to define the topotecan penetration through BTB of gliomas. Our hypothesis is the pathological conditions in glioma will increase topotecan penetration into tumor tissue compared with normal brain. Thus, the objective of this study is to provide insights to the topotecan disposition in a mouse glioma (U-87) model.

\subsection{Methods and materials}

\subsubsection{Drugs and reagents}

Topotecan (Hycamtin ${ }^{\mathrm{TM}}$ ) was obtained from GlaxoSmithKline (Philadelphia, PA) and prepared in sterile water at a concentration of $1 \mathrm{mg} / \mathrm{ml}$. HPLC grade reagents are obtained from Fisher Scientific (Pittsburgh, PA) or Sigma-Aldrich (St. Louis, MO).

\subsubsection{Tumor cell line}

Firefly luciferase labeled U-87 human glioblastoma cells were kindly provided by Dr. Davidoff (Department of Surgery, St. Jude Children's Research Hospital). Cells were cultured in DMEM medium (10-013-CM, Mediatech, Herndorn, VA) supplemented with 10\% fetal bovine serum (SV30014.03, Hyclone, Logan UT), 1\% L-glutamine (13533, Invitrogen, Grand Island, NY) and $0.4 \mathrm{mg} / \mathrm{ml} \mathrm{G} 418$ (10131-035, Invitrogen, Grand Island, $\mathrm{NY}$ ) in a humidified incubator with $5 \% \mathrm{CO}_{2}$ at $37^{\circ} \mathrm{C} .80 \%-90 \%$ confluent cells were collected, counted and resuspended in Matrigel (356234, BD Biosciences, San Jose, CA) to prepare a cell suspension of $1 \times 10^{5}$ cells/ $\mu$ l for tumor inoculation.

\subsubsection{Animal}

CD-1 nude female mice were purchased from Charles River Laboratories

(Wilmington, MA). The mice were maintained on a 12 hour light/dark cycle with free 
access to food and water. After surgery, the mice were housed individually in cages. All procedures were approved by St. Jude Institutional Animal Care and Use Committee and in accordance with the Association for Assessment and Accreditation of Laboratory Animal Care (AALAC).

\subsubsection{Intracranial tumor inoculation}

CD-1 nude female mice (25-30 g) were anaesthetized with ketamine (Abbott Laboratories, Chicago IL) /xylazine (Butler Company, Columbus, OH) at the dosage of one $0.1 \mathrm{ml}$ intraperitoneal injection followed by two $0.05 \mathrm{ml}$ intramuscular injections. The skull was secured in a stereotaxic apparatus. A small piece of scalp was removed to expose the top of skull. A burr hole was drilled (AP: $-2 \mathrm{~mm}$, ML: $1.5 \mathrm{~mm}$, DV: $-2 \mathrm{~mm}$, relative to bregma point on a flat skull). Two additional small holes were drilled for bone anchor screws a few millimeters away from the burr hole. A $25 \mu 1$ Hamilton syringe (14813-21, Fisher) with a 26-gauge needle (14-816-212A, Fisher) was used to implant tumor cells. The syringe was lowered down $2 \mathrm{~mm}$ into the brain parenchyma through the burr hole drilled previously. $2.5 \mu \mathrm{l}$ of tumor cells in Matrigel was slowly injected into the brain. After 1 minute, the syringe was retrieved by $1 \mathrm{~mm}$ and another $2.5 \mu 1$ of tumor cells was injected. After 1 minute the syringe was slowly removed. Total $5 \times 10^{5}$ cells in 5 $\mu 1$ Matrigel were injected.

\subsubsection{Microdialysis guide cannula implant}

Three different groups of study were conducted. 1) For microdialysis study in tumor tissue, a guide cannula (MD-2255, Bioanalytical Systems, West Lafayette, IN) was inserted ( $2 \mathrm{~mm}$ deep) through the same burr hole as the tumor implant right after tumor inoculation. 2) For microdialysis in contralateral normal tissue study, another burr hole was drilled (1.5 mm lateral to the left, $2 \mathrm{~mm}$ posterior) for guide cannula implant. 3) For microdialysis study in nontumor bearing mice, only the matrigel without tumor was sham inoculated. Dental cement was applied to seal the scalp opening and support the anchor screws. Animals were returned to cages and observed for survival.

\subsubsection{Bioluminescence imaging}

Luciferin (69000, Xenogen, Hopkinton, MA) was prepared in water at a concentration of $15 \mathrm{mg} / \mathrm{ml}$ and administered to animals through i.p. (giving $3 \mathrm{mg}$ per mouse). At 4 minutes post-injection, mice were anesthetized using isoflurane and placed into the Xenogen IVIS-200 imaging system where anesthesia was maintained for the duration of the scan (no longer than $5 \mathrm{~min}$ ). After scanning, animals were allowed to recover under observation and supplemented with oxygen where necessary. The total flux (photons/second) from the whole tumor imaged area was used as the bioluminescence signal. 


\subsubsection{Correlation of tumor growth with bioluminescence signal}

24 CD-1 nude female mice were inoculated with luciferase labeled U-87 cells on day 1 as described above (without guide cannula). On day 7, 9, 11, 13, 15, 17 after tumor implant, three or four mice were randomly selected and measured for bioluminescence signals as described above. After imaging the mice were euthanized immediately and the whole head was removed and fixed in 10\% neutral formalin for 48 hours. The mouse brain was then excised and the tumor was measured on three dimensions ( $\mathrm{X}, \mathrm{Y}$ and $\mathrm{Z}$ ). The tumor volume was calculated by using an ellipsoid equation (tumor volume $=X^{*} Y^{*} Z * 0.5233$ ). The tumor volume was correlated to the bioluminescence signal.

\subsubsection{Microdialysis procedure}

CD-1 nude mice inoculated with tumor were monitored and imaged on day 12 or 13 after tumor implant. Mice with the bioluminescence signals within the range of $1 \times 10^{8}$ to $8 \times 10^{8} \mathrm{p} / \mathrm{s}$ were used for microdialysis study. The microdialysis procedure and probe calibration was previously reported in detail (Leggas et al., 2004c; Zhuang et al., 2006). Briefly, on the day of an experiment, a microdialysis probe (MD-2211, Bioanalytical Systems, West Lafayette, IN) was flushed with blank artificial CSF (aCSF, NaCl $148 \mathrm{mM}, \mathrm{KCl} 4 \mathrm{mM}, \mathrm{MgCl}_{2} 0.8 \mathrm{mM}, \mathrm{CaCl}_{2} 1.4 \mathrm{mM}, \mathrm{Na}_{2} \mathrm{HPO}_{4} 1.2 \mathrm{mM}, \mathrm{NaH}_{2} \mathrm{PO}_{4} 0.3 \mathrm{mM}$ and Dextrose $5 \mathrm{mM}, \mathrm{pH}$ adjusted to 7.4 ). The probe was inserted through the cannula into the brain, and the aCSF perfusion rate was set at $0.5 \mu 1 / \mathrm{min}$. The probe was allowed to equilibrate in vivo for 1 hour. Topotecan ( $4 \mathrm{mg} / \mathrm{kg}$ ) was administered by i.v. bolus through the lateral tail vein. The dialysate samples were directly loaded onto a sample loop $(2 \mu \mathrm{l})$ and analyzed through an online microbore HPLC system (Leggas et al., 2004c). Probe recovery was determined using the in vivo retrodialysis method after each experiment was completed. During the microdialysis period, three plasma samples were collected from a mouse at $0.25,1$, and 3 hours after topotecan administration for plasma pharmacokinetic analysis (Zhuang et al., 2006).

\subsubsection{Histology}

At the completion of a microdialysis experiment, the animal was euthanized and the brain removed and fixed in 10\% NBF for at least 48 hours. The brain was then cut into coronal segments, dehydrated, cleared and embedded in a paraffin block. Sections were cut on a microtome at $4 \mu \mathrm{m}$ thickness and every tenth section was collected and stained with haematoxylin and eosin. All sections from a given block were examined microscopically to verify the location of the microdialysis probe via the track in the tissue (Zhuang et al., 2006). 


\subsubsection{Noncompartmental analysis of brain pharmacokinetic data}

The pharmacokinetic analysis of topotecan lactone was performed for each mouse (Zhuang et al., 2006). Topotecan lactone concentrations in vCSF were corrected for the hydrolysis of topotecan lactone to the carboxylate that occurs in $\mathrm{pH} 7.4$ artificial CSF as described previously (Leggas et al., 2004c). The AUC in vCSF from time 0 to infinity was estimated using the linear trapezoidal method with the addition of a residual area as depicted in Equation 4-1,

$$
A U C_{\text {brain }}^{0 \rightarrow \infty}=\sum_{i=1}^{n} \frac{C_{i}+C_{i-1}}{2} * \Delta t+C_{n} / \beta
$$

where $C_{i}$ is the topotecan concentration in dialysate; $\Delta t$ is the sample injection interval; $\mathrm{C}_{\mathrm{n}}$ is the last measurable topotecan concentration; $\beta$ is estimated by linear regression of the logarithm of the last 4-5 measurable concentrations.

\subsubsection{Protein binding}

Topotecan lactone protein binding was determined in plasma collected from CD-1 nude tumor bearing mice. The approach was previously described (Zhuang et al., 2006). Briefly, topotecan lactone was added to the plasma to make a $500 \mathrm{ng} / \mathrm{ml}$ solution. Spiked plasma $(0.5 \mathrm{~mL})$ was added to a Centrifree YM-30 (Millipore Corporation, Billerica, MA) sample reservoir and centrifuged at $2000 \mathrm{~g}$ for 20 minutes. Topotecan was extracted from the protein and ultrafiltrate portion of the device, respectively, by adding $50 \mu \mathrm{L}$ of sample immediately to $200 \mu \mathrm{L}$ of cold methanol stored on dry ice. Samples were analyzed by an HPLC method as previously reported (Furman et al., 1996; Baker et al., 1996). Topotecan lactone fraction unbound was calculated as amount of unbound topotecan lactone divided by total amount of topotecan lactone in plasma.

\subsubsection{Compartmental analysis of plasma pharmacokinetic data}

Plasma topotecan lactone concentration-time data were first corrected for plasma protein binding using an unbound factor. A population pharmacokinetic study was first performed to determine the population mean and variance for unbound topotecan lactone pharmacokinetic parameters as previously described (Zhuang et al., 2006). Then for each mouse, a two-compartment pharmacokinetic model was fitted to the plasma topotecan lactone concentration-time data using maximum a posteriori probability (MAP) Bayesian estimation as implemented in ADAPT II (D'Argenio and Schumitzky, 1979). Pharmacokinetic parameters determined included volume of central compartment $\left(\mathrm{V}_{\mathrm{c}}\right)$, total elimination clearance $\left(\mathrm{CL}_{\mathrm{t}}\right)$, volume of peripheral compartment $\left(\mathrm{V}_{\mathrm{p}}\right)$ and intercompartmental clearance $\left(\mathrm{CL}_{\mathrm{p}}\right)$. Using these parameters, the unbound topotecan lactone concentrations in the plasma were simulated in ADAPT II. The area under the concentration-time curve from zero to infinity $\left(\mathrm{AUC}_{0 \rightarrow \infty}\right)$ for the unbound topotecan plasma was calculated by integration of the simulated concentration-time data from 
model estimates. A ratio of the brain AUC to unbound plasma topotecan lactone AUC was calculated and used as a measurement of topotecan vCSF penetration (HammarlundUdenaes et al., 1997).

\subsubsection{Statistical analysis}

Statistical analysis was performed in Sigma Stat 3.1. One way ANOVA was conducted to determine the significance of between group differences. The difference was considered significant when $\mathrm{p}<0.05$.

\subsection{Results}

\subsubsection{Topotecan lactone protein binding}

Topotecan protein binding was assessed in the plasma collected from CD-1 nude tumor bearing mice. The topotecan lactone unbound fraction was determined $27.0 \% \pm 2.43$.

\subsubsection{Tumor volume correlated to bioluminescence signal}

To assess the correlation between bioluminescence signal and tumor volume, tumor growth was monitored by measuring the bioluminescence signal indicated by total flux (photon/s). After completion of imaging, the tumor volume was measured and then correlated with bioluminescence signal. As shown in Figure 4-1, although the bioluminescence signal increased with the growth of tumor, a linear regression only produced a $\mathrm{R}^{2}=0.47$ Figure 4-2, which indicates the bioluminescence signal is not linearly correlated with tumor volume very well and was only used as a reference.

\subsubsection{Topotecan penetration is not statistically different in the U-87 tumor tissue from normal tissue}

Topotecan penetration was assessed in U-87 tumor tissue, normal tissue of nontumor bearing mice and contralateral normal tissue of tumor bearing mice. The probe placement was verified in the tumor or normal tissue as depicted in Figure 4-3. After one i.v. bolus $4 \mathrm{mg} / \mathrm{kg}$ topotecan injection, the brain ECF to plasma AUC ratio of unbound topotecan lactone was determined as $0.68 \pm 0.32(\mathrm{n}=9), 0.82 \pm 0.47(\mathrm{n}=3)$ and $0.63 \pm 0.59$ $(n=3)$ in tumor tissue, normal brain of non-tumor bearing mice and contralateral normal brain of tumor bearing mice Figure 4-4. No statistical difference was found between any groups (one way ANOVA, $\mathrm{F}=0.182, \mathrm{P}=0.836$ ). 


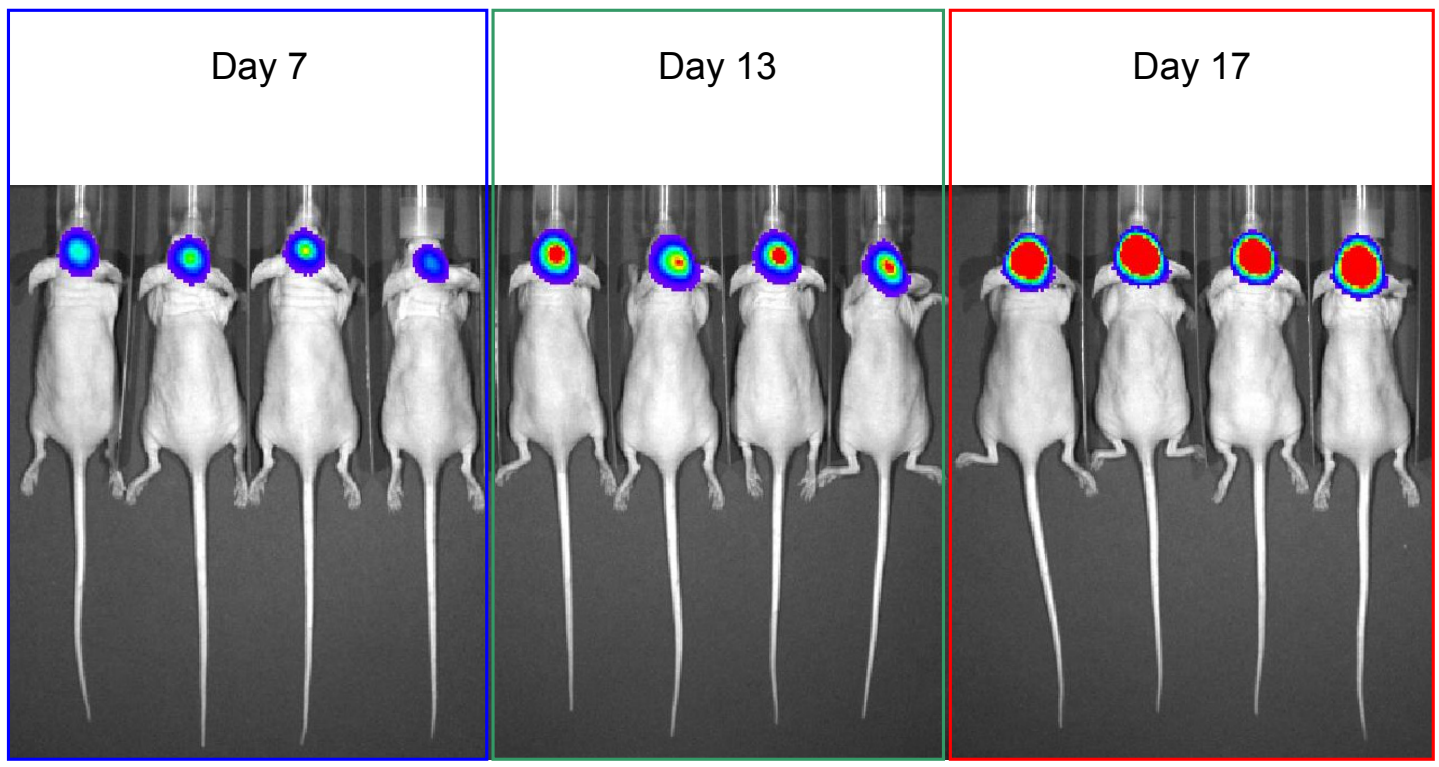

Figure 4-1 Tumor (U-87) growth monitored by bioluminescence.

U-87 tumor was implanted on day 1. Tumor growth was monitored with bioluminescence imaging by randomly selecting 3 to 4 mice from total 24 mice. The signal strength increased from blue to red. The images on day 7,13 and 17 after tumor implant were shown. 


\section{Luminescence vs Tumor volume}

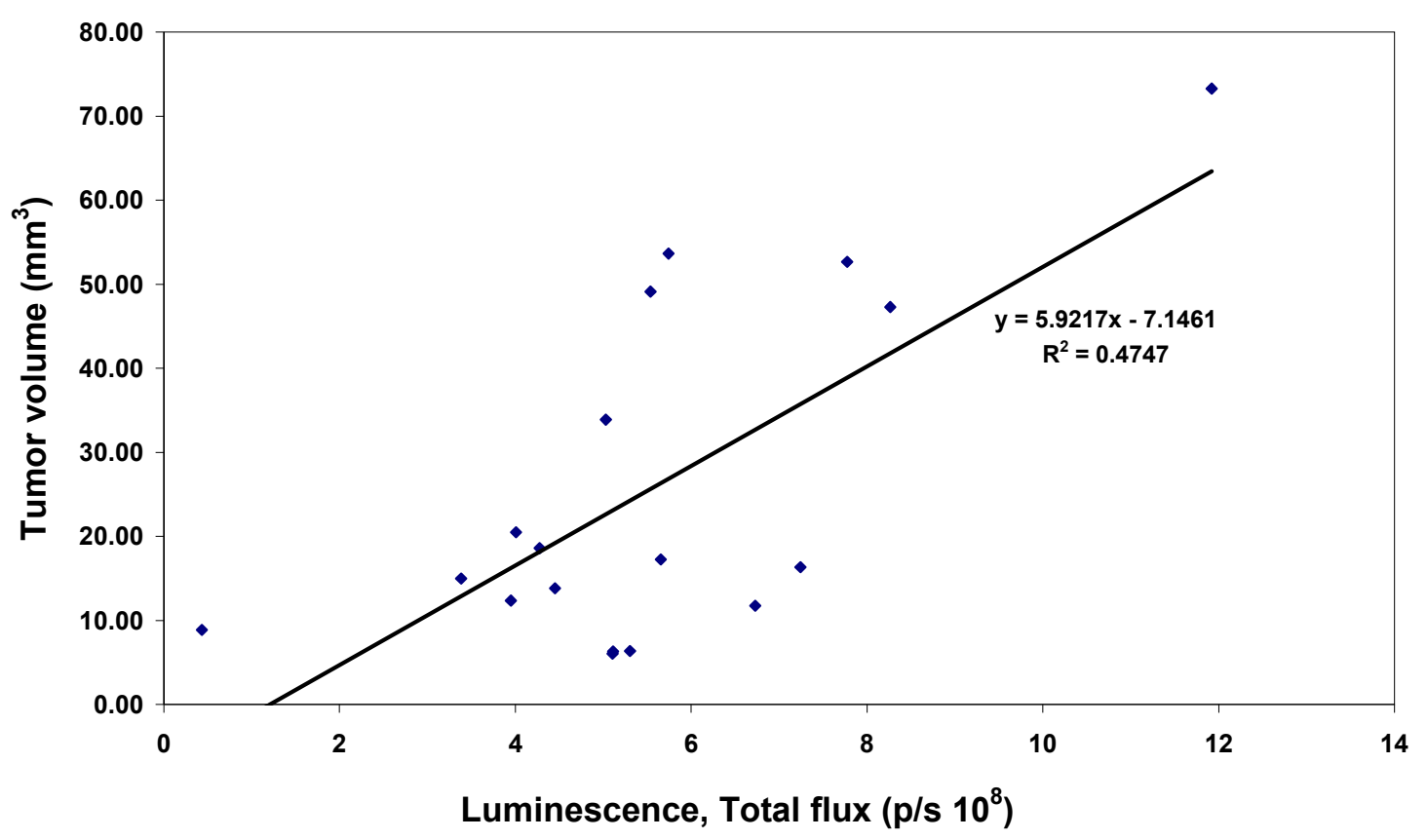

Figure 4-2 Correlation of tumor volume to bioluminescence signal.

A linear regression was performed to correlate the bioluminescence signal (total flux $\mathrm{p} / \mathrm{s}$ $\left.10^{8}\right)$ to tumor volume $\left(\mathrm{mm}^{3}\right)$. 

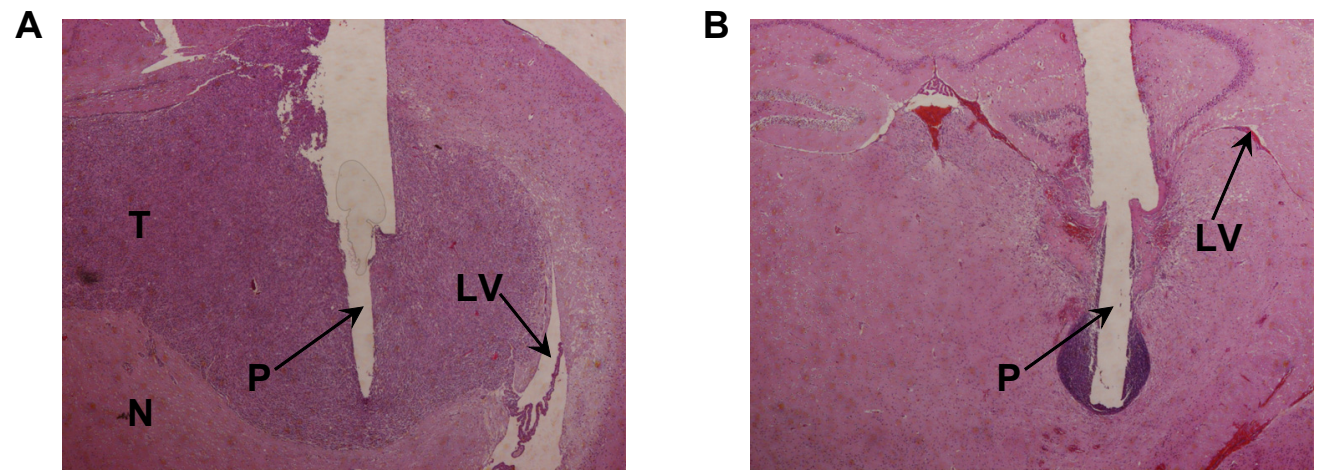

Figure 4-3 Microdialysis probe in tumor or normal brain.

H\&E staining of coronal section of probe insertion into tumor (A) or normal brain (B). Probe $(\mathrm{P})$, lateral ventricle $(\mathrm{LV})$, tumor tissue $(\mathrm{T})$, normal brain $(\mathrm{N})$. 


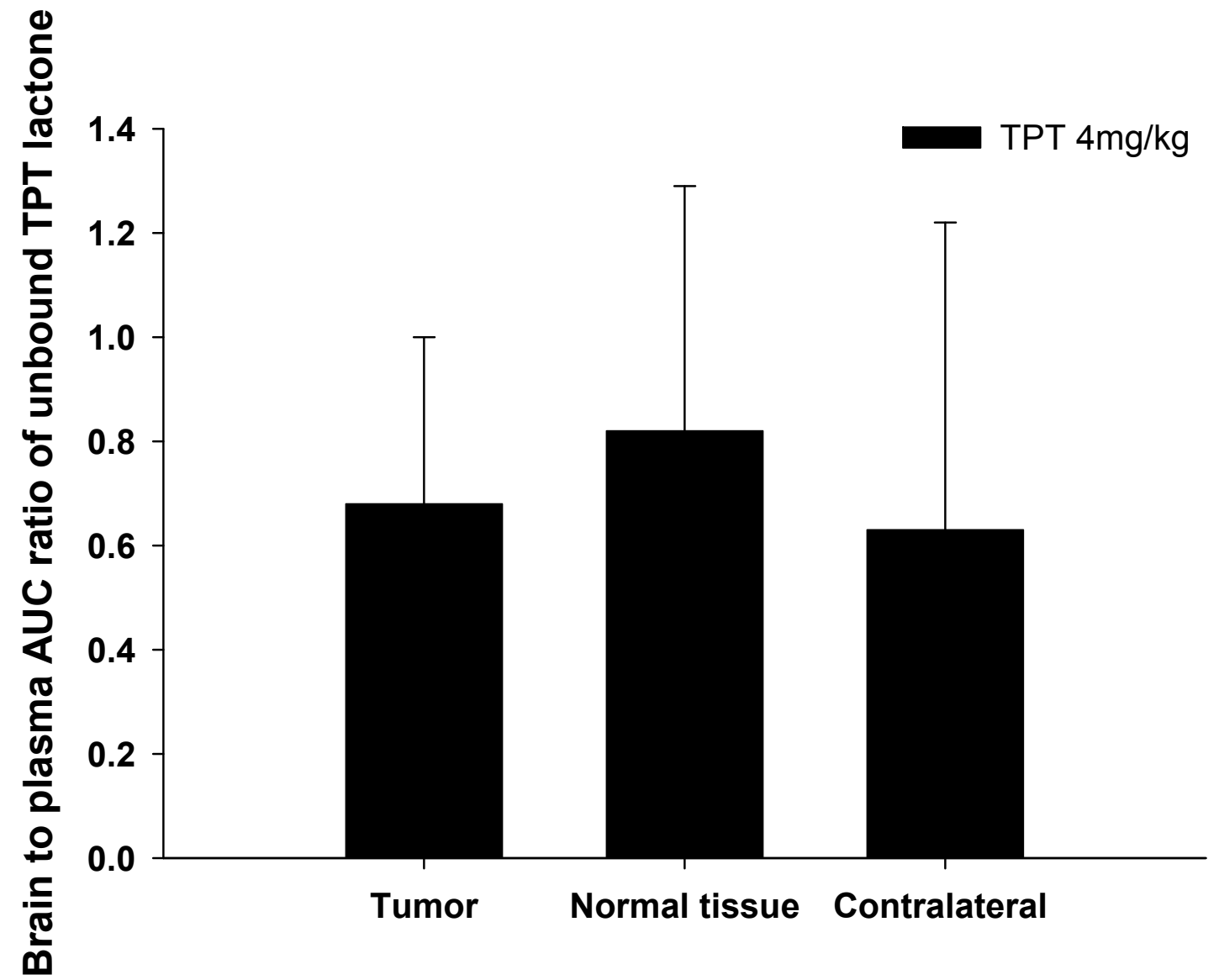

Figure 4-4 TPT penetration in the U-87 tumor model of CD-1 nude mice.

After one i.v. bolus $4 \mathrm{mg} / \mathrm{kg}$ topotecan injection, the brain ECF to plasma AUC ratio of unbound topotecan lactone was determined in tumor tissue (Tumor), normal brain of nontumor bearing mice (Normal tissue) and contralateral normal brain of tumor bearing mice (Contralateral). (one way ANOVA, $\mathrm{F}=0.182, \mathrm{P}>0.05$ ) 


\subsection{Discussion}

Topotecan penetration in gliomas is not well understood. In this study, we constructed an orthotopic human glioblastoma (U-87) xenograft mouse model to gain insights into topotecan penetration into a glioma tumor model, which is primarily parenchymal tissue based. Our preliminary results suggest that topotecan penetration in U-87 glioma tumor tissue is not statistically different from normal brain.

U-87 is a well established human glioblastoma cell line. It has often been used as a human glioma model (Claes et al., 2008; Nakatsu et al., 1997). The tumor line expresses several ABC transporters such as P-gp and MRP1(Rittierodt et al., 2004; Perek et al., 2005). Our previous study also suggested MRP4 might also be expressed in U-87 (unpublished data). In the present study, U-87 was labeled with a firefly luciferase, which makes it possible to monitor the tumor growth with bioluminescence imaging in vivo.

It is also recognized that U-87 grows in a self-contained fashion (Cretu et al., 2005; Ozawa et al., 2002), which does not recapitulate the infiltrative feature of gliomas in clinic. This limitation has to be taken into account in the interpretation of drug penetration data obtained in U-87.

Although topotecan penetration into normal brain tissue ECF was restricted by $\mathrm{ABC}$ transporters (e.g., Bcrp1 and P-gp) as described in Chapter 3, the overall effect of the presence of tumor on topotecan penetration is not clear. Straathof et al. first reported in a rat glioma (9L) model, topotecan concentrations in tumor were 20 -fold higher than in normal brain tissue (Straathof et al., 1999). However, in that study the topotecan concentrations in tumor or normal brain were determined by tissue homogenate method. Furthermore the total concentrations rather than protein unbound concentrations were used. The conclusions based on these data could be misleading due to the limitations of the homogenate method, which included the drug that was bound to proteins (also discussed in Chapter 1).

In the current study, by using intratumoral microdialysis the protein unbound topotecan concentrations were directly measured through an online microbore HPLC system. Plasma concentrations were corrected with a topotecan protein unbound fraction (0.27). The tumor to plasma AUC ratio of unbound topotecan lactone $\left(\mathrm{AUC}_{\mathrm{u}, \text { tumor }} / \mathrm{AUC}_{\mathrm{u} \text {,plasma }}\right)$ serves a more reliable indicator for topotecan intratumoral penetration.

Another anti-cancer drug methotrexate was reported to have a higher penetration in brain tumor than in normal brain by using intratumoral microdialysis (de Lange et al., 1995; Devineni et al., 1996a; Dukic et al., 2004). Based on our data, topotecan apparently has a different disposition tendency in glioma. Thus it is strongly suggested that drug disposition in CNS tumors depend not only on tumor but also on drug properties. The penetration data from one drug cannot be extrapolated to another without care. 
Unexpectedly, the normal brain to plasma AUC ratio of unbound topotecan lactone in CD-1 nude mice $(0.82 \pm 0.47)$ appears much higher than in FVB mice $(0.36 \pm 0.06$, Chapter 3$)$. It might be attributed to the position through which guide cannulas were implanted. Although the microdialysis probe was apparently in the parenchymal tissue, the position we selected in this study went through the lateral ventricle, which might cause CSF to leak into the microdialysis probe. For future study, a new position should be used to implant tumor and a guide cannula to rule out the possibility of CSF contamination. 


\section{Chapter 5. Summary and Future Directions}

Therapy for primary CNS tumors, especially malignant gliomas, still remains a major challenge. Failure of therapy could be attributed to multiple factors, which can be categorized into tumor intrinsic resistance, therapy related induced resistance, and inadequate drug delivery. Camptothecin analogs (e.g. topotecan and irinotecan) demonstrate potent anti-tumor activity including CNS tumors in animal xenograft models. However, the results of clinical trials with camptothecin analogs for CNS tumors are far from satisfactory. Although medulloblastoma patients responded modestly (Stewart et al., 2004a; Nicholson et al., 2007), malignant glioma patients responded poorly (Chintagumpala et al., 2006; Nicholson et al., 2007).

A possible explanation for the discrepancy between the preclinical and clinical results is that in the subcutaneous xenograft tumor model, drug administration is associated with an increased drug delivery to the tumor sites. This is compared with clinical studies where systemic drug delivery to intracranial tumor sites may be inadequate due to two well-known barriers in the brain, BBB and BCB. From the perspective of drug delivery, the $\mathrm{BBB}$ and the $\mathrm{BCB}$ regulate two distinct brain compartments; the ECF compartment of parenchymal tissues and the brain vCSF compartment respectively. After systemic administration, drugs must pass through at least one of these two barriers to gain access to the brain (Loscher and Potschka, 2005a). Included in these barriers are transporter proteins of the ATP binding cassette (ABC) family, including P-gp, MRP1, MRP2, MRP4, and BCRP (Ito et al., 2005b). These transporters play a role in drug resistance and results of several studies have shown that topotecan is a substrate for BCRP (Maliepaard et al., 1999b; Maliepaard et al., 2001b; Ito et al., 2005a), P-gp (Chen et al., 1991; Hendricks et al., 1992a), and MRP4 (Leggas et al., 2004b). The distinct physiological and molecular properties of BBB and BCB could result in very different drug penetration into the two brain compartments (ECF or CSF) through these two barriers (Zhuang et al., 2006). Thus, it is necessary to investigate each compartment by separate experiments. Although some work has been published on the extent to which these barriers regulate topotecan CNS penetration, it would be of great clinical interest to gain a better understanding of how and to what degree this regulation occurs.

Investigation of drug penetration into these compartments may be performed utilizing a microdialysis technique in the ventricular CSF or in ECF of brain parenchymal tissues. To date, most work using this technique has been done in a rodent model, primarily the rat. It has not been common practice to use murine models for microdialysis studies as it poses a great deal of technical challenge to place microdialysis probe precisely into the mouse ventricle due to its small size and strain related variability in anatomy. However the traditional perpendicular probe insertion method used primarily in rats gives a very low success rate and poor reproducibility in murine models. Although we had previously developed a method to perform the microdialysis procedure in FVB mouse, but it was often associated with probe placement failure and side-effects (e.g., hemorrhage, inflammatory reactions) making interpretation of the results difficult. We 
are the first laboratory to study drug distribution in vCSF by using the CNS microdialysis technique in a murine model. Since the current work requires the use of knockout mice, it is necessary to develop a more reliable probe placement procedure to precisely place the microdialysis probe into the ventricle with an acceptable success rate and fewer side effects.

We developed a modified surgical procedure to implant a microdialysis probe to sample vCSF in FVB mice. The modification consisted of changes in the stereotaxic coordinates and insertion of the cannula and ultimately the probe at a 20 degree angle (Shen et al., 2008). Exact placement of the probe was confirmed using ultrasound (US), micro-computed tomography (CT), and histologic review of serial paraffin sections. Additionally, studies of topotecan CSF penetration in the FVB mouse were conducted. Unexpectedly with this modified procedure, the ventricular CSF to plasma AUC ratio of unbound topotecan lactone was greater than that previously reported using conventional methods (Shen et al., 2008). We speculate this is due to changes incorporated by the modified procedure that places the probe directly into the lateral ventricle allowing sampling of that discrete compartment.

Next we applied this modified surgical procedure in the microdialysis studies to investigate the mechanisms that control topotecan penetration into brain ECF or vCSF. A differential topotecan penetration in two CNS compartments, the ventricular cerebrospinal fluid (vCSF) and brain parenchymal extracellular fluid (ECF) was determined in targeted mutation mouse models $\left(\right.$ Bcrpl $^{(-/)}, M d r l a / b^{(-/)}$and Bcrpl/Mdrla/ $\left./ b^{(--)}\right)$. After an intravenous (i.v.) bolus dose of $4 \mathrm{mg} / \mathrm{kg}$ topotecan, unbound lactone vCSF penetration was defined by a vCSF to plasma area under the curve ratio $\left(\mathrm{AUC}_{\mathrm{u}, \mathrm{vCSF}} / \mathrm{AUC}_{\mathrm{u} \text {,plasma }}\right.$; mean $\left.\pm \mathrm{SD}\right)$ of $2.85 \pm 0.14,2.3 \pm 0.12,1.5 \pm 0.05$ and $0.86 \pm 0.05$ in FVB, Mdrla/b(-), Bcrpl $1^{(--)}$and Bcrpl/Mdrla/l $b^{(-/)}$mice, respectively. The penetration into the brain parenchyma, defined by the ECF to plasma AUC ratio $\left(\mathrm{AUC}_{\mathrm{u}, \mathrm{ECF}} / \mathrm{AUC}_{\mathrm{u}, \mathrm{plasma}}\right.$ ) was determined as $0.35 \pm 0.04,0.35 \pm 0.01$ and $0.81 \pm 0.04$ for $\mathrm{FVB}$, Bcrpl $^{(--)}$and Bcrp $1 / M d r l a / b^{(-/)}$mice, respectively. By co-administering the tyrosine kinase inhibitor gefitinib (200 mg/kg) to inhibit Bcrpl and P-gp transporters, the $\mathrm{AUC}_{\mathrm{u}, \mathrm{VCSF}} / \mathrm{AUC}_{\mathrm{u} \text {,plasma }}$ values significantly decreased to $1.15 \pm 0.16$ in FVB mice and $1.1 \pm 0.14$ in $B c r p 1 / M d r l a / b^{(--)}$, while $\mathrm{AUC}_{\mathrm{u}, \mathrm{ECF}} / \mathrm{AUC}_{\mathrm{u} \text {,plasma }}$ ratios significantly increased to $0.63 \pm 0.03$ in FVB and $0.95 \pm 0.02$ in Bcrp1/Mdr1 $\mathrm{a} / \mathrm{b}^{(-/)}$. Interestingly, a preferential disposition of topotecan lactone over carboxylate was apparent in vCSF. The topotecan lactone to carboxylate $\mathrm{AUC}$ ratio in $\mathrm{VCSF}$ ( $\mathrm{AUC}_{\text {lactone, } \mathrm{CSF}} / \mathrm{AUC}_{\text {carboxylate, } \mathrm{CSF}}$ ) was calculated as $5.69 \pm 0.83,3.85 \pm 0.64,3.61 \pm 0.46$ and $0.78 \pm 0.19$ in FVB, $M d r 1 a / b^{(-/)}$, Bcrpl $^{(--)}$and Bcrpl/Mdrla/b $b^{(-/)}$mice, respectively. In contrast, in mouse plasma this lactone to carboxylate ratio did not change significantly between the mouse knockout strains. Taken together, our results 1) support a dominant role of Bcrp1 over P-gp in topotecan penetration through the blood CSF barrier (BCB), 2) suggest that Bcrp1 and Pgp work similarly in constraining topotecan penetration through the blood brain barrier (BBB), and 3) determine, for the first time, that the lactone form is a preferred substrate for the $\mathrm{ABC}$ transporters as compared to the carboxylate. Our findings could be relevant for the improvement of pharmacological strategies to treat brain tumors. 
Although this study was designed to evaluate topotecan CNS penetration, our long-term goal is to establish a model to determine the CNS penetration of any drug, and address research questions that are related to drug transport mechanisms. Such a model must include reliable surgical procedures to target distinct brain compartments (e.g., ventricles or parenchymal tissues), microdialysis sampling technique coupled with a sensitive bioanalytical method, proper animal models, and robust pharmacokinetic analysis method. This study was an example of successful application of such a model for topotecan CNS penetration.

Once we had an understanding of the mechanisms that control topotecan CNS penetration under physiological conditions, we further investigated its penetration in the presence of tumor. A luciferase labeled human glioblastoma (U-87) mouse model was constructed and used in this study. This allowed the tumor growth to be monitored by measuring the bioluminescence signal. Our preliminary results suggest topotecan penetration in the U-87 tumor model was not really different from the normal brain. However this model might be biased due to the location of tumor inoculation, which went through the mouse lateral ventricle. Based on the knowledge obtained from Chapter 3 , the leaking CSF might cause an overestimation of the drug penetration into brain parenchyma. Further investigation is necessary.

Evidence has just started to accumulate that drug differential penetration into ECF from CSF may be a more universal phenomenon in more species. A Pfizer group reported that in rats an investigational new drug CP-615003 had a CSF-to serum ratio seven times as high as ECF-to serum ratio (Venkatakrishnan et al., 2007). An HIV-1 proteinase inhibitor nelfinavir, a P-gp substrate, was also reported to have distinct penetration into CSF from ECF in nonhuman primates. With co-treatment of a P-gp inhibitor zosuquidar, nelfinavir ECF concentrations were increased by 146-fold while the CSF concentrations were unaffected (Kaddoumi et al., 2007). Most recently it was reported that P-gp restricted loperamide penetration into ECF, but not in CSF, in a spontaneous canine Pglycoprotein knockout model (Mealey et al., 2008). Thus, for future studies, the tumor inoculation position in the brain should be adjusted where a microdialysis probe does not have to go through the lateral ventricle. This is particularly important for drugs (e.g., topotecan) that have distinct penetration into ECF from CSF.

Over the last decade many genetic manipulated mouse models have been generated to mimic human CNS tumors (e.g., medulloblastoma and glioblastoma) (Uziel et al., 2005; Romer et al., 2004; Lee et al., 2003). In addition to xenograft models, the mouse spontaneous tumor models are better alternatives to investigate drug penetration into the tumors. As spontaneous models offer an in vivo tumor environment with much less artificial effects, which are important for drug penetration studies.

With appropriate tumor models, research questions with great clinical relevance can be addressed. For example, in clinical practice many CNS tumor patients also receive dexamethasone for controlling the brain edema (Nahaczewski et al., 2004).

Dexamethasone (DEX), a synthetic glucocorticoid, binds to glucocorticoid receptor that is widely expressed intracellularly and regulates transcription of various genes involved 
in glucose metabolism, cytochrome P450 drug metabolizing enzymes, drug transporters and immune response. Numerous studies have demonstrated DEX can enhance CYP3As expression in rodents (Anakk et al., 2003; Down et al., 2006; Wei et al., 2002; Huss and Kasper, 2000) and human (Moore et al., 2000; Pascussi et al., 2000b; Pascussi et al., 2000a; Pascussi et al., 2001) through nuclear receptor PXR. DEX also regulates drug transporters expression and the pattern is more complicated. In a few early studies the effects of DEX treatment on P-gp are not consistent. A murine thymoma cell line was induced to overexpress P-gp by DEX (Bourgeois et al., 1993). In rat hepatocytes P-gp can only be weakly upregulated by DEX (Fardel et al., 1993). Later on it became clearer that the P-gp regulation by DEX could be cell and tissue specific. In primary normal rat hepatocytes DEX suppressed P-gp expression (Chieli et al., 1994; Schuetz et al., 1995) through a post-transcriptional mechanism. However in a rat hepatoma cell line H35 DEX activated P-gp transcription (Schuetz et al., 1995). A recent study in rats has clearly demonstrated DEX treatment significantly upregulated P-gp mRNA and protein by 5 7 fold in small intestine but down-regulated in liver by $90 \%$ and little or no change in kidney, colon or brain microvessels (Mei et al., 2004). However another study in a rat endothelial cell line showed even the protein level of P-gp was not changed by DEX, Pgp function was actually enhanced by a decreased accumulation of vincristine assay (Regina et al., 1999). DEX also upregulates MRP2 in both human and rat hepatocytes (Courtois et al., 1999; Johnson and Klaassen, 2002; Kast et al., 2002; Kubitz et al., 1999). Most recently DEX was shown to induce BCRP in the rat brain microvessels (unpublished data). Since CYP3A, P-gp and BCRP are all involved in TPT metabolism or elimination, the differential regulatory effects of DEX on these proteins make it hard to predict the overall effect of DEX on TPT. On the other hand TPT exposure is deeply related to response, a small reduction in TPT systemic exposure may lead to a significant loss of antitumor activity (Furman et al., 1996). Recently DEX has also been shown to confer resistance to cisplatin and 5-fluorouracil in many of the malignant tumor cells (Zhang et al., 2006). Thus it would be of great clinical interest to understand how cotreatment of dexamethasone affects anti-cancer drug disposition and metabolism.

Different strategies to enhance drug penetration (e.g., $\mathrm{ABC}$ transporter modulators, anti-angiogenesis therapy) can also be tested in these tumor models. Once the pharmacokinetics in tumor tissue can be defined in mouse models, a pharmacokinetic model can then be developed to relate the drug exposure in tumor to the exposure in plasma. With the model, once an effective therapy is found for a specific tumor in mice, it can be translated for human clinical trials. 


\section{List of References}

Abbott NJ, 2002. Astrocyte-endothelial interactions and blood-brain barrier permeability. J. Anat. 200: 629-638.

Abbott NJ, 2005. Dynamics of CNS barriers: evolution, differentiation, and modulation. Cell Mol. Neurobiol. 25: 5-23.

Al-Mefty O, Topsakal C, Pravdenkova S, Sawyer JR, Harrison MJ, 2004. Radiationinduced meningiomas: clinical, pathological, cytokinetic, and cytogenetic characteristics. J. Neurosurg. 100: 1002-1013.

Allen JD, Brinkhuis RF, Wijnholds J, Schinkel AH, 1999. The mouse Bcrp1/Mxr/Abcp gene: amplification and overexpression in cell lines selected for resistance to topotecan, mitoxantrone, or doxorubicin. Cancer Res. 59: 4237-4241.

American Cancer Society. Detailed Guide: Brain / CNS Tumors in Children. 2007. Ref Type: Generic

Anakk S, Kalsotra A, Shen Q, Vu MT, Staudinger JL, Davies PJ, Strobel HW, 2003. Genomic characterization and regulation of CYP3a13: role of xenobiotics and nuclear receptors. FASEB J. 17: 1736-1738.

Apparaju SK, Gudelsky GA, Desai PB, 2008. Pharmacokinetics of gemcitabine in tumor and non-tumor extracellular fluid of brain: an in vivo assessment in rats employing intracerebral microdialysis. Cancer Chemother. Pharmacol. 61: 223-229.

Arismendi-Morillo G, Castellano A, 2005. Tumoral micro-blood vessels and vascular microenvironment in human astrocytic tumors. A transmission electron microscopy study. J Neurooncol. 73: 211-217.

Arner P, Bolinder J, Eliasson A, Lundin A, Ungerstedt U, 1988. Microdialysis of adipose tissue and blood for in vivo lipolysis studies. Am. J. Physiol 255: E737-E742.

Arroyo AG, Genis L, Gonzalo P, Matias-Roman S, Pollan A, Galvez BG, 2007. Matrix metalloproteinases: new routes to the use of MT1-MMP as a therapeutic target in angiogenesis-related disease. Curr. Pharm. Des 13: 1787-1802.

Asthagiri AR, Pouratian N, Sherman J, Ahmed G, Shaffrey ME, 2007. Advances in brain tumor surgery. Neurol. Clin. 25: 975-1003.

Baker SD, Heideman RL, Crom WR, Kuttesch JF, Gajjar A, Stewart CF, 1996.

Cerebrospinal fluid pharmacokinetics and penetration of continuous infusion topotecan in children with central nervous system tumors. Cancer Chemother. Pharmacol. 37: 195202. 
Balabanov R, Dore-Duffy P, 1998. Role of the CNS microvascular pericyte in the bloodbrain barrier. J. Neurosci. Res. 53: 637-644.

Baluk P, Hashizume H, McDonald DM, 2005. Cellular abnormalities of blood vessels as targets in cancer. Curr. Opin. Genet. Dev. 15: 102-111.

Batchelor TT, Gilbert MR, Supko JG, Carson KA, Nabors LB, Grossman SA, Lesser GJ, Mikkelsen T, Phuphanich S, 2004. Phase 2 study of weekly irinotecan in adults with recurrent malignant glioma: final report of NABTT 97-11. Neuro. -oncol. 6: 21-27.

Ben-Nun J, Cooper RL, Cringle SJ, Constable IJ, 1988. Ocular dialysis. A new technique for in vivo intraocular pharmacokinetic measurements. Arch. Ophthalmol. 106: 254-259.

Benveniste H, Drejer J, Schousboe A, Diemer NH, 1987. Regional cerebral glucose phosphorylation and blood flow after insertion of a microdialysis fiber through the dorsal hippocampus in the rat. J. Neurochem. 49: 729-734.

Benveniste H, Huttemeier PC, 1990. Microdialysis--theory and application. Prog. Neurobiol. 35: 195-215.

Bernier-Chastagner V, Grill J, Doz F, Bracard S, Gentet JC, Marie-Cardine A, Luporsi E, Margueritte G, Lejars O, Laithier V, Mechinaud F, Millot F, Kalifa C, Chastagner P, 2005. Topotecan as a radiosensitizer in the treatment of children with malignant diffuse brainstem gliomas: results of a French Society of Paediatric Oncology Phase II Study. Cancer 104: 2792-2797.

Bito L, Davson H, Levin E, Murray M, Snider N, 1966. The concentrations of free amino acids and other electrolytes in cerebrospinal fluid, in vivo dialysate of brain, and blood plasma of the dog. J. Neurochem. 13: 1057-1067.

Blaney SM, Cole DE, Balis FM, Godwin K, Poplack DG, 1993. Plasma and cerebrospinal fluid pharmacokinetic study of topotecan in nonhuman primates. Cancer Res. 53: 725-727.

Blaney SM, Phillips PC, Packer RJ, Heideman RL, Berg SL, Adamson PC, Allen JC, Sallan SE, Jakacki RI, Lange BJ, Reaman GH, Horowitz ME, Poplack DG, Balis FM, 1996. Phase II evaluation of topotecan for pediatric central nervous system tumors. Cancer 78: 527-531.

Blaney SM, Takimoto C, Murry DJ, Kuttesch N, McCully C, Cole DE, Godwin K, Balis FM, 1998. Plasma and cerebrospinal fluid pharmacokinetics of 9-aminocamptothecin (9$\mathrm{AC})$, irinotecan (CPT-11), and SN-38 in nonhuman primates. Cancer Chemother. Pharmacol. 41: 464-468.

Bleyer WA, 1999. Epidemiologic impact of children with brain tumors. Childs Nerv. Syst. 15: 758-763. 
Bomgaars LR, Bernstein M, Krailo M, Kadota R, Das S, Chen Z, Adamson PC, Blaney SM, 2007. Phase II trial of irinotecan in children with refractory solid tumors: a Children's Oncology Group Study. J. Clin. Oncol. 25: 4622-4627.

Boschi G, Launay N, Rips R, 1981. Implantation of an intracerebral cannula in the mouse. J. Pharmacol. Methods 6: 193-198.

Boschi G, Scherrmann J, 2000. Microdialysis in mice for drug delivery research. Adv. Drug Deliv. Rev. 45: 271-281.

Bostrom E, Simonsson US, Hammarlund-Udenaes M, 2006. In vivo blood-brain barrier transport of oxycodone in the rat: indications for active influx and implications for pharmacokinetics/pharmacodynamics. Drug Metab Dispos. 34: 1624-1631.

Bourgeois S, Gruol DJ, Newby RF, Rajah FM, 1993. Expression of an mdr gene is associated with a new form of resistance to dexamethasone-induced apoptosis. Mol. Endocrinol. 7: 840-851.

Brangi M, Litman T, Ciotti M, Nishiyama K, Kohlhagen G, Takimoto C, Robey R, Pommier Y, Fojo T, Bates SE, 1999. Camptothecin resistance: role of the ATP-binding cassette (ABC), mitoxantrone-resistance half-transporter (MXR), and potential for glucuronidation in MXR-expressing cells. Cancer Res. 59: 5938-5946.

Broniscer A, Gururangan S, MacDonald TJ, Goldman S, Packer RJ, Stewart CF, Wallace D, Danks MK, Friedman HS, Poussaint TY, Kun LE, Boyett JM, Gajjar A, 2007. Phase I trial of single-dose temozolomide and continuous administration of o6-benzylguanine in children with brain tumors: a pediatric brain tumor consortium report. Clin. Cancer Res. 13: 6712-6718.

Capranico G, Ferri F, Fogli MV, Russo A, Lotito L, Baranello L, 2007. The effects of camptothecin on RNA polymerase II transcription: roles of DNA topoisomerase I.

Biochimie 89: 482-489.

Carmeliet P, Jain RK, 2000. Angiogenesis in cancer and other diseases. Nature 407: 249257.

Castro MG, Cowen R, Williamson IK, David A, Jimenez-Dalmaroni MJ, Yuan X, Bigliari A, Williams JC, Hu J, Lowenstein PR, 2003. Current and future strategies for the treatment of malignant brain tumors. Pharmacol. Ther. 98: 71-108.

Centers for Disease Control and Prevention, 2007. Trends in childhood cancer mortality-United States, 1990-2004. MMWR Morb. Mortal. Wkly. Rep. 56: 1257-1261.

Chamberlain MC, 2002. Salvage chemotherapy with CPT-11 for recurrent glioblastoma multiforme. J. Neurooncol. 56: 183-188. 
Chen AY, Yu C, Potmesil M, Wall ME, Wani MC, Liu LF, 1991. Camptothecin overcomes MDR1-mediated resistance in human KB carcinoma cells. Cancer Res. 51: 6039-6044.

Chieli E, Santoni-Rugiu E, Cervelli F, Sabbatini A, Petrini M, Romiti N, Paolicchi A, Tongiani R, 1994. Differential modulation of P-glycoprotein expression by dexamethasone and 3-methylcholanthrene in rat hepatocyte primary cultures. Carcinogenesis 15: 335-341.

Chintagumpala MM, Friedman HS, Stewart CF, Kepner J, McLendon RE, Modrich PL, McCluggage C, Burger P, Holmes E, Thompson S, Rutka J, Michalski J, Woo S, Blaney SM, Kun LE, Horowitz ME, 2006. A phase II window trial of procarbazine and topotecan in children with high-grade glioma: a report from the children's oncology group. J. Neurooncol. 77: 193-198.

Chu XY, Kato Y, Sugiyama Y, 1999a. Possible involvement of P-glycoprotein in biliary excretion of CPT-11 in rats. Drug Metab Dispos. 27: 440-441.

Chu XY, Suzuki H, Ueda K, Kato Y, Akiyama S, Sugiyama Y, 1999b. Active efflux of CPT-11 and its metabolites in human KB-derived cell lines. J. Pharmacol. Exp. Ther. 288: 735-741.

Cirrito JR, May PC, O'Dell MA, Taylor JW, Parsadanian M, Cramer JW, Audia JE, Nissen JS, Bales KR, Paul SM, DeMattos RB, Holtzman DM, 2003. In vivo assessment of brain interstitial fluid with microdialysis reveals plaque-associated changes in amyloid-beta metabolism and half-life. J. Neurosci. 23: 8844-8853.

Cisternino S, Mercier C, Bourasset F, Roux F, Scherrmann JM, 2004. Expression, upregulation, and transport activity of the multidrug-resistance protein Abcg2 at the mouse blood-brain barrier. Cancer Res. 64: 3296-3301.

Claes A, Wesseling P, Jeuken J, Maass C, Heerschap A, Leenders WP, 2008. Antiangiogenic compounds interfere with chemotherapy of brain tumors due to vessel normalization. Mol. Cancer Ther. 7: 71-78.

Clark WG, Vivonia CA, Baxter CF, 1968. Accurate freehand injection into the lateral brain ventricle of the conscious mouse. J. Appl. Physiol 25: 319-321.

Cloughesy TF, Filka E, Nelson G, Kabbinavar F, Friedman H, Miller LL, Elfring GL, 2002. Irinotecan treatment for recurrent malignant glioma using an every-3-week regimen. Am. J. Clin. Oncol. 25: 204-208.

Colorado PC, Torre A, Kamphaus G, Maeshima Y, Hopfer H, Takahashi K, Volk R, Zamborsky ED, Herman S, Sarkar PK, Ericksen MB, Dhanabal M, Simons M, Post M, Kufe DW, Weichselbaum RR, Sukhatme VP, Kalluri R, 2000. Anti-angiogenic cues from vascular basement membrane collagen. Cancer Res. 60: 2520-2526. 
Cooray HC, Blackmore CG, Maskell L, Barrand MA, 2002. Localisation of breast cancer resistance protein in microvessel endothelium of human brain. Neuroreport 13: 20592063.

Cordon-Cardo C, O'Brien JP, Boccia J, Casals D, Bertino JR, Melamed MR, 1990. Expression of the multidrug resistance gene product (P-glycoprotein) in human normal and tumor tissues. J. Histochem. Cytochem. 38: 1277-1287.

Courtois A, Payen L, Guillouzo A, Fardel O, 1999. Up-regulation of multidrug resistance-associated protein 2 (MRP2) expression in rat hepatocytes by dexamethasone. FEBS Lett. 459: 381-385.

Cretu A, Fotos JS, Little BW, Galileo DS, 2005. Human and rat glioma growth, invasion, and vascularization in a novel chick embryo brain tumor model. Clin. Exp. Metastasis 22: $225-236$.

D'Argenio DZ, Schumitzky A, 1979. A program package for simulation and parameter estimation in pharmacokinetic systems. Comput. Programs Biomed. 9: 115-134.

Davson H, Segal M, 1996. Physiology of the CSF and blood-brain barriers. CRC Press Inc., Boca Raton, FL.

de Lange EC, Danhof M, de Boer AG, Breimer DD, 1997. Methodological considerations of intracerebral microdialysis in pharmacokinetic studies on drug transport across the blood-brain barrier. Brain Res. Brain Res. Rev. 25: 27-49.

de Lange EC, de Boer AG, Breimer DD, 2000. Methodological issues in microdialysis sampling for pharmacokinetic studies. Adv. Drug Deliv. Rev. 45: 125-148.

de Lange EC, de Vries JD, Zurcher C, Danhof M, de Boer AG, Breimer DD, 1995. The use of intracerebral microdialysis for the determination of pharmacokinetic profiles of anticancer drugs in tumor-bearing rat brain. Pharm. Res. 12: 1924-1931.

de Lange EC, de BG, Schinkel AH, de Boer AG, Breimer DD, 1998. BBB transport and P-glycoprotein functionality using MDR1A (-/-) and wild-type mice. Total brain versus microdialysis concentration profiles of rhodamine-123. Pharm. Res. 15: 1657-1665.

de Vries NA, Zhao J, Kroon E, Buckle T, Beijnen JH, van TO, 2007. P-glycoprotein and breast cancer resistance protein: two dominant transporters working together in limiting the brain penetration of topotecan. Clin. Cancer Res. 13: 6440-6449.

Deguchi Y, 2002. Application of in vivo brain microdialysis to the study of blood-brain barrier transport of drugs. Drug Metab Pharmacokinet. 17: 395-407.

Dempsey EW, Wislocki GB, 1955. An electron microscopic study of the blood-brain barrier in the rat, employing silver nitrate as a vital stain. J. Biophys. Biochem. Cytol. 1: $245-256$. 
Denny WA, Rewcastle GW, Bridges AJ, Fry DW, Kraker AJ, 1996. Structure-activity relationships for 4-anilinoquinazolines as potent inhibitors at the ATP binding site of the epidermal growth factor receptor in vitro. Clin. Exp. Pharmacol. Physiol 23: 424-427.

Desrayaud S, Guntz P, Scherrmann JM, Lemaire M, 1997. Effect of the P-glycoprotein inhibitor, SDZ PSC 833, on the blood and brain pharmacokinetics of colchicine. Life Sci. 61: 153-163.

Devineni D, Klein-Szanto A, Gallo JM, 1996a. In vivo microdialysis to characterize drug transport in brain tumors: analysis of methotrexate uptake in rat glioma-2 (RG-2)-bearing rats. Cancer Chemother. Pharmacol. 38: 499-507.

Devineni D, Klein-Szanto A, Gallo JM, 1996b. Uptake of temozolomide in a rat glioma model in the presence and absence of the angiogenesis inhibitor TNP-470. Cancer Res. 56: 1983-1987.

Dickson PV, Hamner JB, Sims TL, Fraga CH, Ng CY, Rajasekeran S, Hagedorn NL, McCarville MB, Stewart CF, Davidoff AM, 2007. Bevacizumab-induced transient remodeling of the vasculature in neuroblastoma xenografts results in improved delivery and efficacy of systemically administered chemotherapy. Clin. Cancer Res. 13: 39423950.

Down MJ, Arkle S, Mills JJ, 2006. Regulation and induction of CYP3A11, CYP3A13 and CYP3A25 in C57BL/6J mouse liver. Arch. Biochem. Biophys.

Dukic SF, Kaltenbach ML, Heurtaux T, Hoizey G, Lallemand A, Vistelle R, 2004. Influence of C6 and CNS1 brain tumors on methotrexate pharmacokinetics in plasma and brain tissue. J. Neurooncol. 67: 131-138.

Eisenblatter T, Huwel S, Galla HJ, 2003. Characterisation of the brain multidrug resistance protein (BMDP/ABCG2/BCRP) expressed at the blood-brain barrier. Brain Res. 971: 221-231.

Elmquist WF, Sawchuk RJ, 2000. Use of microdialysis in drug delivery studies. Adv. Drug Deliv. Rev. 45: 123-124.

Evans AE, Jenkin RD, Sposto R, Ortega JA, Wilson CB, Wara W, Ertel IJ, Kramer S, Chang CH, Leikin SL, ., 1990. The treatment of medulloblastoma. Results of a prospective randomized trial of radiation therapy with and without $\mathrm{CCNU}$, vincristine, and prednisone. J. Neurosurg. 72: 572-582.

Faraci FM, Kinzenbaw D, Heistad DD, 1994. Effect of endogenous vasopressin on blood flow to choroid plexus during hypoxia and intracranial hypertension. Am. J. Physiol 266: H393-H398.

Fardel O, Lecureur V, Guillouzo A, 1993. Regulation by dexamethasone of Pglycoprotein expression in cultured rat hepatocytes. FEBS Lett. 327: 189-193. 
Ferrara N, 2004. Vascular endothelial growth factor: basic science and clinical progress. Endocr. Rev. 25: 581-611.

Finlay JL, 1999. Chemotherapy for childhood brain tumors: an appraisal for the millenium. Childs Nerv. Syst. 15: 496-497.

Fisher JL, Schwartzbaum JA, Wrensch M, Wiemels JL, 2007. Epidemiology of brain tumors. Neurol. Clin. 25: 867-90, vii.

Folkman J, 1971. Tumor angiogenesis: therapeutic implications. N. Engl. J. Med. 285: 1182-1186.

Franklin KB, 1997. The mouse brain in stereotaxic coordinates. Academic Press, San Diego, CA 92101.

Friedman HS, Colvin OM, Ludeman SM, Schold SC, Jr., Boyd VL, Mulhbaier LH, Bigner DD, 1986. Experimental chemotherapy of human medulloblastoma with classical alkylators. Cancer Res. 46: 2827-2833.

Friedman HS, Houghton PJ, Schold SC, Keir S, Bigner DD, 1994. Activity of 9dimethylaminomethyl-10-hydroxycamptothecin against pediatric and adult central nervous system tumor xenografts. Cancer Chemother. Pharmacol. 34: 171-174.

Friedman HS, Kerby T, Fields S, Zilisch JE, Graden D, McLendon RE, Houghton PJ, Arbuck S, Cokgor I, Friedman AH, 1999a. Topotecan treatment of adults with primary malignant glioma. The Brain Tumor Center at Duke. Cancer 85: 1160-1165.

Friedman HS, Petros WP, Friedman AH, Schaaf LJ, Kerby T, Lawyer J, Parry M, Houghton PJ, Lovell S, Rasheed K, Cloughsey T, Stewart ES, Colvin OM, Provenzale JM, McLendon RE, Bigner DD, Cokgor I, Haglund M, Rich J, Ashley D, Malczyn J, Elfring GL, Miller LL, 1999b. Irinotecan therapy in adults with recurrent or progressive malignant glioma. J. Clin. Oncol. 17: 1516-1525.

Friedman HS, Schold SC, Jr., Varia M, Bigner DD, 1983. Chemotherapy and radiation therapy of human medulloblastoma in athymic nude mice. Cancer Res. 43: 3088-3093.

Furman WL, Baker SD, Pratt CB, Rivera GK, Evans WE, Stewart CF, 1996. Escalating systemic exposure of continuous infusion topotecan in children with recurrent acute leukemia. J. Clin. Oncol. 14: 1504-1511.

Gajjar A, Chintagumpala M, Ashley D, Kellie S, Kun LE, Merchant TE, Woo S, Wheeler G, Ahern V, Krasin MJ, Fouladi M, Broniscer A, Krance R, Hale GA, Stewart CF, Dauser R, Sanford RA, Fuller C, Lau C, Boyett JM, Wallace D, Gilbertson RJ, 2006. Risk-adapted craniospinal radiotherapy followed by high-dose chemotherapy and stemcell rescue in children with newly diagnosed medulloblastoma (St Jude Medulloblastoma-96): long-term results from a prospective, multicentre trial. Lancet Oncol. 7: 813-820. 
Gao B, Stieger B, Noe B, Fritschy JM, Meier PJ, 1999. Localization of the organic anion transporting polypeptide 2 (Oatp2) in capillary endothelium and choroid plexus epithelium of rat brain. J. Histochem. Cytochem. 47: 1255-1264.

Ghersi-Egea JF, Minn A, Siest G, 1988. A new aspect of the protective functions of the blood-brain barrier: activities of four drug-metabolizing enzymes in isolated rat brain microvessels. Life Sci. 42: 2515-2523.

Gilbert MR, Supko JG, Batchelor T, Lesser G, Fisher JD, Piantadosi S, Grossman S, 2003. Phase I clinical and pharmacokinetic study of irinotecan in adults with recurrent malignant glioma. Clin. Cancer Res. 9: 2940-2949.

Gottardo NG, Gajjar A, 2006. Current Therapy for Medulloblastoma. Curr. Treat. Options. Neurol. 8: 319-334.

Gottesman MM, Fojo T, Bates SE, 2002. Multidrug resistance in cancer: role of ATPdependent transporters. Nat. Rev. Cancer 2: 48-58.

Groothuis DR, Fischer JM, Lapin G, Bigner DD, Vick NA, 1982. Permeability of different experimental brain tumor models to horseradish peroxidase. J. Neuropathol. Exp. Neurol. 41: 164-185.

Groothuis DR, Fischer JM, Pasternak JF, Blasberg RG, Vick NA, Bigner DD, 1983. Regional measurements of blood-to-tissue transport in experimental RG-2 rat gliomas. Cancer Res. 43: 3368-3373.

Groothuis DR, Molnar P, Blasberg RG, 1984. Regional blood flow and blood-to-tissue transport in five brain tumor models. Implications for chemotherapy. Prog. Exp. Tumor Res. 27: 132-153.

Gupta A, Chatelain P, Massingham R, Jonsson EN, Hammarlund-Udenaes M, 2006. Brain distribution of cetirizine enantiomers: comparison of three different tissue-toplasma partition coefficients: K(p), K(p,u), and K(p,uu). Drug Metab Dispos. 34: 318323.

Gururangan S, Friedman HS, 2004. Recent advances in the treatment of pediatric brain tumors. Oncology (Williston. Park) 18: 1649-1661.

Haley TJ, Mccormick WG, 1957. Pharmacological effects produced by intracerebral injection of drugs in the conscious mouse. Br. J. Pharmacol. Chemother. 12: 12-15.

Hamano Y, Zeisberg M, Sugimoto H, Lively JC, Maeshima Y, Yang C, Hynes RO, Werb Z, Sudhakar A, Kalluri R, 2003. Physiological levels of tumstatin, a fragment of collagen IV alpha3 chain, are generated by MMP-9 proteolysis and suppress angiogenesis via alphaV beta3 integrin. Cancer Cell 3: 589-601. 
Hammarlund-Udenaes M, Paalzow LK, de Lange EC, 1997. Drug equilibration across the blood-brain barrier--pharmacokinetic considerations based on the microdialysis method. Pharm. Res. 14: 128-134.

Hare CB, Elion GB, Houghton PJ, Houghton JA, Keir S, Marcelli SL, Bigner DD, Friedman HS, 1997. Therapeutic efficacy of the topoisomerase I inhibitor 7-ethyl-10-(4[1-piperidino]-1-piperidino)-carbonyloxy-camptothecin against pediatric and adult central nervous system tumor xenografts. Cancer Chemother. Pharmacol. 39: 187-191.

Hasegawa H, Ushio Y, Hayakawa T, Yamada K, Mogami H, 1983. Changes of the blood-brain barrier in experimental metastatic brain tumors. J. Neurosurg. 59: 304-310.

Hashizume H, Baluk P, Morikawa S, McLean JW, Thurston G, Roberge S, Jain RK, McDonald DM, 2000. Openings between defective endothelial cells explain tumor vessel leakiness. Am. J. Pathol. 156: 1363-1380.

Hashizume K, Black KL, 2002. Increased endothelial vesicular transport correlates with increased blood-tumor barrier permeability induced by bradykinin and leukotriene C4. J. Neuropathol. Exp. Neurol. 61: 725-735.

Hedaya MA, Pan WJ, 1997. Effect of alcohol coadministration on the plasma and brain concentrations of cocaine in rats. Drug Metab Dispos. 25: 647-650.

Hediger MA, Romero MF, Peng JB, Rolfs A, Takanaga H, Bruford EA, 2004. The ABCs of solute carriers: physiological, pathological and therapeutic implications of human membrane transport proteinsIntroduction. Pflugers Arch. 447: 465-468.

Hendricks CB, Rowinsky EK, Grochow LB, Donehower RC, Kaufmann SH, 1992b. Effect of P-glycoprotein expression on the accumulation and cytotoxicity of topotecan (SK\&F 104864), a new camptothecin analogue. Cancer Res. 52: 2268-2278.

Hendricks CB, Rowinsky EK, Grochow LB, Donehower RC, Kaufmann SH, 1992a. Effect of P-glycoprotein expression on the accumulation and cytotoxicity of topotecan (SK\&F 104864), a new camptothecin analogue. Cancer Res. 52: 2268-2278.

Hernandez L, Stanley BG, Hoebel BG, 1986. A small, removable microdialysis probe. Life Sci. 39: 2629-2637.

Hertzberg RP, Caranfa MJ, Holden KG, Jakas DR, Gallagher G, Mattern MR, Mong SM, Bartus JO, Johnson RK, Kingsbury WD, 1989. Modification of the hydroxy lactone ring of camptothecin: inhibition of mammalian topoisomerase I and biological activity. $\mathrm{J}$. Med. Chem. 32: 715-720.

Hori S, Ohtsuki S, Tachikawa M, Kimura N, Kondo T, Watanabe M, Nakashima E, Terasaki T, 2004. Functional expression of rat ABCG2 on the luminal side of brain capillaries and its enhancement by astrocyte-derived soluble factor(s). J. Neurochem. 90: 526-536. 
Houghton PJ, Cheshire PJ, Hallman JD, Lutz L, Friedman HS, Danks MK, Houghton JA, 1995. Efficacy of topoisomerase I inhibitors, topotecan and irinotecan, administered at low dose levels in protracted schedules to mice bearing xenografts of human tumors. Cancer Chemother. Pharmacol. 36: 393-403.

Huss JM, Kasper CB, 2000. Two-stage glucocorticoid induction of CYP3A23 through both the glucocorticoid and pregnane X receptors. Mol. Pharmacol. 58: 48-57.

Ito K, Suzuki H, Horie T, Sugiyama Y, 2005a. Apical/basolateral surface expression of drug transporters and its role in vectorial drug transport. Pharm. Res. 22: 1559-1577.

Ito K, Suzuki H, Horie T, Sugiyama Y, 2005b. Apical/basolateral surface expression of drug transporters and its role in vectorial drug transport. Pharm. Res. 22: 1559-1577.

Ito K, Suzuki H, Horie T, Sugiyama Y, 2005c. Apical/basolateral surface expression of drug transporters and its role in vectorial drug transport. Pharm. Res. 22: 1559-1577.

Jain RK, 1989. Delivery of novel therapeutic agents in tumors: physiological barriers and strategies. J. Natl. Cancer Inst. 81: 570-576.

Jemal A, Siegel R, Ward E, Murray T, Xu J, Thun MJ, 2007. Cancer statistics, 2007. CA Cancer J. Clin. 57: 43-66.

Johanson CE, Duncan JA, Stopa EG, Baird A, 2005. Enhanced prospects for drug delivery and brain targeting by the choroid plexus-CSF route. Pharm. Res. 22: 10111037.

Johnson DR, Klaassen CD, 2002. Regulation of rat multidrug resistance protein 2 by classes of prototypical microsomal enzyme inducers that activate distinct transcription pathways. Toxicol. Sci. 67: 182-189.

Johnson MD, Anderson BD, 1996. Localization of purine metabolizing enzymes in bovine brain microvessel endothelial cells: an enzymatic blood-brain barrier for dideoxynucleosides? Pharm. Res. 13: 1881-1886.

Jonker JW, Buitelaar M, Wagenaar E, van d, V, Scheffer GL, Scheper RJ, Plosch T, Kuipers F, Elferink RP, Rosing H, Beijnen JH, Schinkel AH, 2002. The breast cancer resistance protein protects against a major chlorophyll-derived dietary phototoxin and protoporphyria. Proc. Natl. Acad. Sci. U. S. A 99: 15649-15654.

Jonker JW, Freeman J, Bolscher E, Musters S, Alvi AJ, Titley I, Schinkel AH, Dale TC, 2005. Contribution of the ABC transporters Bcrp1 and Mdrla/1b to the side population phenotype in mammary gland and bone marrow of mice. Stem Cells 23: 1059-1065.

Juliano RL, Ling V, 1976. A surface glycoprotein modulating drug permeability in Chinese hamster ovary cell mutants. Biochim. Biophys. Acta 455: 152-162. 
Kaddoumi A, Choi SU, Kinman L, Whittington D, Tsai CC, Ho RJ, Anderson BD, Unadkat JD, 2007. Inhibition of P-glycoprotein activity at the primate blood-brain barrier increases the distribution of nelfinavir into the brain but not into the cerebrospinal fluid. Drug Metab Dispos. 35: 1459-1462.

Kalluri R, 2003. Basement membranes: structure, assembly and role in tumour angiogenesis. Nat. Rev. Cancer 3: 422-433.

Kamphaus GD, Colorado PC, Panka DJ, Hopfer H, Ramchandran R, Torre A, Maeshima Y, Mier JW, Sukhatme VP, Kalluri R, 2000. Canstatin, a novel matrix-derived inhibitor of angiogenesis and tumor growth. J. Biol. Chem. 275: 1209-1215.

Kast HR, Goodwin B, Tarr PT, Jones SA, Anisfeld AM, Stoltz CM, Tontonoz P, Kliewer S, Willson TM, Edwards PA, 2002. Regulation of multidrug resistance-associated protein 2 (ABCC2) by the nuclear receptors pregnane $\mathrm{X}$ receptor, farnesoid $\mathrm{X}$-activated receptor, and constitutive androstane receptor. J. Biol. Chem. 277: 2908-2915.

Keep RF, Jones HC, 1990. A morphometric study on the development of the lateral ventricle choroid plexus, choroid plexus capillaries and ventricular ependyma in the rat. Brain Res Dev. Brain Res 56: 47-53.

Kepler JA, Wani MC, McNaull JN, Wall ME, Levine SG, 1969. Plant antitumor agents. IV. An approach toward the synthesis of camptothecin. J. Org. Chem. 34: 3853-3858.

Kitazaki T, Oka M, Nakamura Y, Tsurutani J, Doi S, Yasunaga M, Takemura M, Yabuuchi H, Soda H, Kohno S, 2005. Gefitinib, an EGFR tyrosine kinase inhibitor, directly inhibits the function of P-glycoprotein in multidrug resistant cancer cells. Lung Cancer 49: 337-343.

Kohn MI, Tanna NK, Herman GT, Resnick SM, Mozley PD, Gur RE, Alavi A, Zimmerman RA, Gur RC, 1991. Analysis of brain and cerebrospinal fluid volumes with MR imaging. Part I. Methods, reliability, and validation. Radiology 178: 115-122.

Krischer JP, Ragab AH, Kun L, Kim TH, Laurent JP, Boyett JM, Cornell CJ, Link M, Luthy AR, Camitta B, 1991. Nitrogen mustard, vincristine, procarbazine, and prednisone as adjuvant chemotherapy in the treatment of medulloblastoma. A Pediatric Oncology Group study. J. Neurosurg. 74: 905-909.

Kubitz R, Warskulat U, Schmitt M, Haussinger D, 1999. Dexamethasone- and osmolarity-dependent expression of the multidrug-resistance protein 2 in cultured rat hepatocytes. Biochem. J. 340 ( Pt 3): 585-591.

Kudelka AP, Tresukosol D, Edwards CL, Freedman RS, Levenback C, Chantarawiroj P, Gonzalez de LC, Kim EE, Madden T, Wallin B, Hord M, Verschraegen C, Raber M, Kavanagh JJ, 1996. Phase II study of intravenous topotecan as a 5-day infusion for refractory epithelial ovarian carcinoma. J. Clin. Oncol. 14: 1552-1557. 
Kuppens IE, Witteveen EO, Jewell RC, Radema SA, Paul EM, Mangum SG, Beijnen JH, Voest EE, Schellens JH, 2007. A phase I, randomized, open-label, parallel-cohort, dosefinding study of elacridar (GF120918) and oral topotecan in cancer patients. Clin. Cancer Res. 13: 3276-3285.

Lamers LM, Stupp R, van den Bent MJ, Al MJ, Gorlia T, Wasserfallen JB, Mittmann N, Jin SS, Crott R, Uyl-de Groot CA, 2008. Cost-effectiveness of temozolomide for the treatment of newly diagnosed glioblastoma multiforme : a report from the EORTC 26981/22981 NCI-C CE3 Intergroup Study. Cancer.

Lansiaux A, Leonce S, Kraus-Berthier L, Bal-Mahieu C, Mazinghien R, Didier S, vidCordonnier MH, Hautefaye P, Lavielle G, Bailly C, Hickman JA, Pierre A, 2007. Novel stable camptothecin derivatives replacing the E-ring lactone by a ketone function are potent inhibitors of topoisomerase I and promising antitumor drugs. Mol. Pharmacol. 72: 311-319.

Lee Y, Miller HL, Jensen P, Hernan R, Connelly M, Wetmore C, Zindy F, Roussel MF, Curran T, Gilbertson RJ, McKinnon PJ, 2003. A molecular fingerprint for medulloblastoma. Cancer Res. 63: 5428-5437.

Leggas M, Adachi M, Scheffer GL, Sun D, Wielinga P, Du G, Mercer KE, Zhuang Y, Panetta JC, Johnston B, Scheper RJ, Stewart CF, Schuetz JD, 2004a. Mrp4 confers resistance to topotecan and protects the brain from chemotherapy. Mol. Cell Biol. 24: $7612-7621$.

Leggas M, Adachi M, Scheffer GL, Sun D, Wielinga P, Du G, Mercer KE, Zhuang Y, Panetta JC, Johnston B, Scheper RJ, Stewart CF, Schuetz JD, 2004b. Mrp4 confers resistance to topotecan and protects the brain from chemotherapy. Mol. Cell Biol. 24: $7612-7621$.

Leggas M, Panetta JC, Zhuang Y, Schuetz JD, Johnston B, Bai F, Sorrentino B, Zhou S, Houghton PJ, Stewart CF, 2006. Gefitinib modulates the function of multiple ATPbinding cassette transporters in vivo. Cancer Res. 66: 4802-4807.

Leggas M, Zhuang Y, Welden J, Self Z, Waters CM, Stewart CF, 2004c. Microbore HPLC method with online microdialysis for measurement of topotecan lactone and carboxylate in murine CSF. J. Pharm. Sci. 93: 2284-2295.

Lehmann A, 1989. Effects of microdialysis-perfusion with anisoosmotic media on extracellular amino acids in the rat hippocampus and skeletal muscle. J. Neurochem. 53: $525-535$.

Levine JE, Powell KD, 1989. Microdialysis for measurement of neuroendocrine peptides. Methods Enzymol. 168: 166-181.

Liebner S, Fischmann A, Rascher G, Duffner F, Grote EH, Kalbacher H, Wolburg H, 2000. Claudin-1 and claudin-5 expression and tight junction morphology are altered in blood vessels of human glioblastoma multiforme. Acta Neuropathol. (Berl) 100: 323-331. 
Loscher W, Potschka H, 2005a. Drug resistance in brain diseases and the role of drug efflux transporters. Nat. Rev. Neurosci. 6: 591-602.

Loscher W, Potschka H, 2005b. Role of drug efflux transporters in the brain for drug disposition and treatment of brain diseases. Prog. Neurobiol. 76: 22-76.

Lynch TJ, Jr., Kalish L, Strauss G, Elias A, Skarin A, Shulman LN, Posner M, Frei E, III, 1994. Phase II study of topotecan in metastatic non-small-cell lung cancer. J. Clin. Oncol. 12: 347-352.

Macdonald D, Cairncross G, Stewart D, Forsyth P, Sawka C, Wainman N, Eisenhauer E, 1996. Phase II study of topotecan in patients with recurrent malignant glioma. National Clinical Institute of Canada Clinical Trials Group. Ann. Oncol. 7: 205-207.

Maeshima Y, Sudhakar A, Lively JC, Ueki K, Kharbanda S, Kahn CR, Sonenberg N, Hynes RO, Kalluri R, 2002. Tumstatin, an endothelial cell-specific inhibitor of protein synthesis. Science 295: 140-143.

Maliepaard M, Scheffer GL, Faneyte IF, van Gastelen MA, Pijnenborg AC, Schinkel AH, van D, V, Scheper RJ, Schellens JH, 2001a. Subcellular localization and distribution of the breast cancer resistance protein transporter in normal human tissues. Cancer Res. 61: 3458-3464.

Maliepaard M, van Gastelen MA, de Jong LA, Pluim D, van Waardenburg RC, Ruevekamp-Helmers MC, Floot BG, Schellens JH, 1999a. Overexpression of the BCRP/MXR/ABCP gene in a topotecan-selected ovarian tumor cell line. Cancer Res. 59: 4559-4563.

Maliepaard M, van Gastelen MA, de Jong LA, Pluim D, van Waardenburg RC, Ruevekamp-Helmers MC, Floot BG, Schellens JH, 1999b. Overexpression of the $\mathrm{BCRP} / \mathrm{MXR} / \mathrm{ABCP}$ gene in a topotecan-selected ovarian tumor cell line. Cancer Res. 59: 4559-4563.

Maliepaard M, van Gastelen MA, Tohgo A, Hausheer FH, van Waardenburg RC, de Jong LA, Pluim D, Beijnen JH, Schellens JH, 2001c. Circumvention of breast cancer resistance protein (BCRP)-mediated resistance to camptothecins in vitro using nonsubstrate drugs or the BCRP inhibitor GF120918. Clin. Cancer Res. 7: 935-941.

Maliepaard M, van Gastelen MA, Tohgo A, Hausheer FH, van Waardenburg RC, de Jong LA, Pluim D, Beijnen JH, Schellens JH, 2001b. Circumvention of breast cancer resistance protein (BCRP)-mediated resistance to camptothecins in vitro using nonsubstrate drugs or the BCRP inhibitor GF120918. Clin. Cancer Res. 7: 935-941.

Marchand C, Antony S, Kohn KW, Cushman M, Ioanoviciu A, Staker BL, Burgin AB, Stewart L, Pommier Y, 2006. A novel norindenoisoquinoline structure reveals a common interfacial inhibitor paradigm for ternary trapping of the topoisomerase I-DNA covalent complex. Mol. Cancer Ther. 5: 287-295. 
Matsumoto SI, Yoshida K, Ishiguro N, Maeda T, Tamai I, 2007. Involvement of Rat and Human Organic Anion Transporter 3 in the Renal Tubular Secretion of Topotecan. J. Pharmacol. Exp. Ther.

Mealey KL, Greene S, Bagley R, Gay JM, Tucker RL, Gavin PR, Schmidt K, Nelson FR, 2008. P-glycoprotein contributes to the blood-brain, but not blood-CSF, barrier in a spontaneous canine P-glycoprotein knockout model. Drug Metab Dispos.

Mei Q, Richards K, Strong-Basalyga K, Fauty SE, Taylor A, Yamazaki M, Prueksaritanont T, Lin JH, Hochman J, 2004. Using real-time quantitative TaqMan RTPCR to evaluate the role of dexamethasone in gene regulation of rat P-glycoproteins mdr1a/1b and cytochrome P450 3A1/2. J. Pharm. Sci. 93: 2488-2496.

Meirieu O, Pairet M, Sutra JF, Ruckebusch M, 1986. Local release of monoamines in the gastrointestinal tract: an in vivo study in rabbits. Life Sci. 38: 827-834.

Merchant TE, Kun LE, Krasin MJ, Wallace D, Chintagumpala MM, Woo SY, Ashley DM, Sexton M, Kellie SJ, Ahern V, Gajjar A, 2007. Multi-Institution Prospective Trial of Reduced-Dose Craniospinal Irradiation (23.4 Gy) Followed by Conformal Posterior Fossa (36 Gy) and Primary Site Irradiation (55.8 Gy) and Dose-Intensive Chemotherapy for Average-Risk Medulloblastoma. Int. J. Radiat. Oncol. Biol. Phys.

Moore LB, Parks DJ, Jones SA, Bledsoe RK, Consler TG, Stimmel JB, Goodwin B, Liddle C, Blanchard SG, Willson TM, Collins JL, Kliewer SA, 2000. Orphan nuclear receptors constitutive androstane receptor and pregnane $\mathrm{X}$ receptor share xenobiotic and steroid ligands. J. Biol. Chem. 275: 15122-15127.

Motl S, Zhuang Y, Waters CM, Stewart CF, 2006. Pharmacokinetic considerations in the treatment of CNS tumours. Clin. Pharmacokinet. 45: 871-903.

Nabors LB, Gillespie GY, Harkins L, King PH, 2001. HuR, a RNA stability factor, is expressed in malignant brain tumors and binds to adenine- and uridine-rich elements within the 3' untranslated regions of cytokine and angiogenic factor mRNAs. Cancer Res. 61: 2154-2161.

Nagata Y, Kusuhara H, Endou H, Sugiyama Y, 2002. Expression and functional characterization of rat organic anion transporter 3 (rOat3) in the choroid plexus. Mol. Pharmacol. 61: 982-988.

Nahaczewski AE, Fowler SB, Hariharan S, 2004. Dexamethasone therapy in patients with brain tumors--a focus on tapering. J. Neurosci. Nurs. 36: 340-343.

Nakamura Y, Oka M, Soda H, Shiozawa K, Yoshikawa M, Itoh A, Ikegami Y, Tsurutani J, Nakatomi K, Kitazaki T, Doi S, Yoshida H, Kohno S, 2005. Gefitinib ("Iressa", ZD1839), an epidermal growth factor receptor tyrosine kinase inhibitor, reverses breast cancer resistance protein/ABCG2-mediated drug resistance. Cancer Res. 65: 1541-1546. 
Nakashima M, Shibata S, Tokunaga Y, Fujita H, Anda T, Arizono K, Tomiyama N, Sasaki H, Ichikawa M, 1997. In-vivo microdialysis study of the distribution of cisplatin into brain tumour tissue after intracarotid infusion in rats with 9L malignant glioma. J. Pharm. Pharmacol. 49: 777-780.

Nakatsu S, Kondo S, Kondo Y, Yin D, Peterson JW, Kaakaji R, Morimura T, Kikuchi H, Takeuchi J, Barnett GH, 1997. Induction of apoptosis in multi-drug resistant (MDR) human glioblastoma cells by SN-38, a metabolite of the camptothecin derivative CPT-11. Cancer Chemother. Pharmacol. 39: 417-423.

Nguyen TD, DeAngelis LM, 2007. Brain metastases. Neurol. Clin. 25: 1173-11xi.

Nicholson HS, Kretschmar CS, Krailo M, Bernstein M, Kadota R, Fort D, Friedman H, Harris MB, Tedeschi-Blok N, Mazewski C, Sato J, Reaman GH, 2007. Phase 2 study of temozolomide in children and adolescents with recurrent central nervous system tumors: a report from the Children's Oncology Group. Cancer 110: 1542-1550.

Nierenberg D, Harbaugh R, Maurer LH, Reeder T, Scott G, Fratkin J, Newman E, 1991. Continuous intratumoral infusion of methotrexate for recurrent glioblastoma: a pilot study. Neurosurgery 28: 752-761.

Nies AT, Jedlitschky G, Konig J, Herold-Mende C, Steiner HH, Schmitt HP, Keppler D, 2004. Expression and immunolocalization of the multidrug resistance proteins, MRP1MRP6 (ABCC1-ABCC6), in human brain. Neuroscience 129: 349-360.

Ningaraj NS, Rao M, Hashizume K, Asotra K, Black KL, 2002. Regulation of bloodbrain tumor barrier permeability by calcium-activated potassium channels. J. Pharmacol. Exp. Ther. 301: 838-851.

Ningaraj NS, Rao MK, Black KL, 2003. Adenosine 5'-triphosphate-sensitive potassium channel-mediated blood-brain tumor barrier permeability increase in a rat brain tumor model. Cancer Res. 63: 8899-8911.

Norris MD, Smith J, Tanabe K, Tobin P, Flemming C, Scheffer GL, Wielinga P, Cohn SL, London WB, Marshall GM, Allen JD, Haber M, 2005. Expression of multidrug transporter MRP4/ABCC4 is a marker of poor prognosis in neuroblastoma and confers resistance to irinotecan in vitro. Mol. Cancer Ther. 4: 547-553.

Nozawa T, Minami H, Sugiura S, Tsuji A, Tamai I, 2005. Role of organic anion transporter OATP1B1 (OATP-C) in hepatic uptake of irinotecan and its active metabolite, 7-ethyl-10-hydroxycamptothecin: in vitro evidence and effect of single nucleotide polymorphisms. Drug Metab Dispos. 33: 434-439.

O'Reilly MS, Boehm T, Shing Y, Fukai N, Vasios G, Lane WS, Flynn E, Birkhead JR, Olsen BR, Folkman J, 1997. Endostatin: an endogenous inhibitor of angiogenesis and tumor growth. Cell 88: 277-285. 
O'Reilly MS, Holmgren L, Shing Y, Chen C, Rosenthal RA, Cao Y, Moses M, Lane WS, Sage EH, Folkman J, 1994. Angiostatin: a circulating endothelial cell inhibitor that suppresses angiogenesis and tumor growth. Cold Spring Harb. Symp. Quant. Biol. 59: 471-482.

Ohtsuki S, Takizawa T, Takanaga H, Hori S, Hosoya K, Terasaki T, 2004. Localization of organic anion transporting polypeptide 3 (oatp3) in mouse brain parenchymal and capillary endothelial cells. J. Neurochem. 90: 743-749.

Ohtsuki S, Terasaki T, 2007. Contribution of carrier-mediated transport systems to the blood-brain barrier as a supporting and protecting interface for the brain; importance for CNS drug discovery and development. Pharm. Res. 24: 1745-1758.

Oldendorf WH, Cornford ME, Brown WJ, 1977. The large apparent work capability of the blood-brain barrier: a study of the mitochondrial content of capillary endothelial cells in brain and other tissues of the rat. Ann. Neurol. 1: 409-417.

Ozawa T, Wang J, Hu LJ, Bollen AW, Lamborn KR, Deen DF, 2002. Growth of human glioblastomas as xenografts in the brains of athymic rats. In Vivo 16: 55-60.

Packer RJ, Gajjar A, Vezina G, Rorke-Adams L, Burger PC, Robertson PL, Bayer L, LaFond D, Donahue BR, Marymont MH, Muraszko K, Langston J, Sposto R, 2006. Phase III study of craniospinal radiation therapy followed by adjuvant chemotherapy for newly diagnosed average-risk medulloblastoma. J. Clin. Oncol. 24: 4202-4208.

Palmieri D, Chambers AF, Felding-Habermann B, Huang S, Steeg PS, 2007. The biology of metastasis to a sanctuary site. Clin. Cancer Res. 13: 1656-1662.

Papadopoulos MC, Saadoun S, Woodrow CJ, Davies DC, Costa-Martins P, Moss RF, Krishna S, Bell BA, 2001. Occludin expression in microvessels of neoplastic and nonneoplastic human brain. Neuropathol. Appl. Neurobiol. 27: 384-395.

Pardridge WM, Oldendorf WH, Cancilla P, Frank HJ, 1986. Blood-brain barrier: interface between internal medicine and the brain. Ann. Intern. Med. 105: 82-95.

Pascussi JM, Drocourt L, Fabre JM, Maurel P, Vilarem MJ, 2000a. Dexamethasone induces pregnane $\mathrm{X}$ receptor and retinoid $\mathrm{X}$ receptor-alpha expression in human hepatocytes: synergistic increase of CYP3A4 induction by pregnane X receptor activators. Mol. Pharmacol. 58: 361-372.

Pascussi JM, Drocourt L, Gerbal-Chaloin S, Fabre JM, Maurel P, Vilarem MJ, 2001. Dual effect of dexamethasone on CYP3A4 gene expression in human hepatocytes. Sequential role of glucocorticoid receptor and pregnane X receptor. Eur. J. Biochem. 268: 6346-6358.

Pascussi JM, Gerbal-Chaloin S, Fabre JM, Maurel P, Vilarem MJ, 2000 b.

Dexamethasone enhances constitutive androstane receptor expression in human 
hepatocytes: consequences on cytochrome P450 gene regulation. Mol. Pharmacol. 58: 1441-1450.

Patel RR, Mehta MP, 2007. Targeted therapy for brain metastases: improving the therapeutic ratio. Clin. Cancer Res. 13: 1675-1683.

Pawlik CA, Houghton PJ, Stewart CF, Cheshire PJ, Richmond LB, Danks MK, 1998. Effective schedules of exposure of medulloblastoma and rhabdomyosarcoma xenografts to topotecan correlate with in vitro assays. Clin. Cancer Res. 4: 1995-2002.

Perek N, Le JN, Denoyer D, Dubois F, 2005. MRP-1 protein expression and glutathione content of in vitro tumor cell lines derived from human glioma carcinoma U-87-MG do not interact with 99mTc-glucarate uptake. Cancer Biother. Radiopharm. 20: 391-400.

Pomeroy SL, Tamayo P, Gaasenbeek M, Sturla LM, Angelo M, McLaughlin ME, Kim JY, Goumnerova LC, Black PM, Lau C, Allen JC, Zagzag D, Olson JM, Curran T, Wetmore C, Biegel JA, Poggio T, Mukherjee S, Rifkin R, Califano A, Stolovitzky G, Louis DN, Mesirov JP, Lander ES, Golub TR, 2002. Prediction of central nervous system embryonal tumour outcome based on gene expression. Nature 415: 436-442.

Potschka H, Fedrowitz M, Loscher W, 2003. Multidrug resistance protein MRP2 contributes to blood-brain barrier function and restricts antiepileptic drug activity. J. Pharmacol. Exp. Ther. 306: 124-131.

Prados MD, Yung WK, Jaeckle KA, Robins HI, Mehta MP, Fine HA, Wen PY, Cloughesy TF, Chang SM, Nicholas MK, Schiff D, Greenberg HS, Junck L, Fink KL, Hess KR, Kuhn J, 2004. Phase 1 trial of irinotecan (CPT-11) in patients with recurrent malignant glioma: a North American Brain Tumor Consortium study. Neuro. -oncol. 6: 44-54.

Rabbani G, Benzil D, Wallam MN, Chen B, Hoang A, Kancherla R, Ahmed T, 2007. Combination therapy with thalidomide, temozolomide and tamoxifen improves quality of life in patients with malignant astrocytomas. Anticancer Res. 27: 2729-2736.

Rao VV, Dahlheimer JL, Bardgett ME, Snyder AZ, Finch RA, Sartorelli AC, PiwnicaWorms D, 1999. Choroid plexus epithelial expression of MDR1 P glycoprotein and multidrug resistance-associated protein contribute to the blood-cerebrospinal-fluid drugpermeability barrier. Proc. Natl. Acad. Sci. U. S. A 96: 3900-3905.

Raymond E, Fabbro M, Boige V, Rixe O, Frenay M, Vassal G, Faivre S, Sicard E, Germa C, Rodier JM, Vernillet L, Armand JP, 2003. Multicentre phase II study and pharmacokinetic analysis of irinotecan in chemotherapy-naive patients with glioblastoma. Ann. Oncol. 14: 603-614.

Redzic ZB, Segal MB, 2004. The structure of the choroid plexus and the physiology of the choroid plexus epithelium. Adv. Drug Deliv. Rev. 56: 1695-1716. 
Regina A, Romero IA, Greenwood J, Adamson P, Bourre JM, Couraud PO, Roux F, 1999. Dexamethasone regulation of P-glycoprotein activity in an immortalized rat brain endothelial cell line, GPNT. J. Neurochem. 73: 1954-1963.

Rittierodt M, Tschernig T, Harada K, 2004. Modulation of multidrug-resistanceassociated P-glycoprotein in human U-87 MG and HUV-ECC cells with antisense oligodeoxynucleotides to MDR1 mRNA. Pathobiology 71: 123-128.

Robertson PL, 2006. Advances in treatment of pediatric brain tumors. NeuroRx. 3: 276291.

Romer JT, Kimura H, Magdaleno S, Sasai K, Fuller C, Baines H, Connelly M, Stewart CF, Gould S, Rubin LL, Curran T, 2004. Suppression of the Shh pathway using a small molecule inhibitor eliminates medulloblastoma in Ptc1(+/-)p53(-/-) mice. Cancer Cell 6: 229-240.

Rothenberg ML, Eckardt JR, Kuhn JG, Burris HA, III, Nelson J, Hilsenbeck SG, Rodriguez GI, Thurman AM, Smith LS, Eckhardt SG, Weiss GR, Elfring GL, Rinaldi DA, Schaaf LJ, Von Hoff DD, 1996. Phase II trial of irinotecan in patients with progressive or rapidly recurrent colorectal cancer. J. Clin. Oncol. 14: 1128-1135.

Rundhaug JE, 2005. Matrix metalloproteinases and angiogenesis. J. Cell Mol. Med. 9: 267-285.

Rutkowski S, Bode U, Deinlein F, Ottensmeier H, Warmuth-Metz M, Soerensen N, Graf N, Emser A, Pietsch T, Wolff JE, Kortmann RD, Kuehl J, 2005. Treatment of early childhood medulloblastoma by postoperative chemotherapy alone. N. Engl. J. Med. 352: 978-986.

Sanders RP, Kocak M, Burger PC, Merchant TE, Gajjar A, Broniscer A, 2007. Highgrade astrocytoma in very young children. Pediatr. Blood Cancer 49: 888-893.

Sasaki Y, Ohtsu A, Shimada Y, Ono K, Saijo N, 1994. Simultaneous administration of CPT-11 and fluorouracil: alteration of the pharmacokinetics of CPT-11 and SN-38 in patients with advanced colorectal cancer. J. Natl. Cancer Inst. 86: 1096-1098.

Sawada T, Kato Y, Kobayashi M, Takekekawa Y, 2000. Immunohistochemical study of tight junction-related protein in neovasculature in astrocytic tumor. Brain Tumor Pathol. 17: 1-6.

Schinkel AH, Mayer U, Wagenaar E, Mol CA, van DL, Smit JJ, van der Valk MA, Voordouw AC, Spits H, van TO, Zijlmans JM, Fibbe WE, Borst P, 1997. Normal viability and altered pharmacokinetics in mice lacking mdr1-type (drug-transporting) Pglycoproteins. Proc. Natl. Acad. Sci. U. S. A 94: 4028-4033.

Schinkel AH, Smit JJ, van TO, Beijnen JH, Wagenaar E, van DL, Mol CA, van d, V, Robanus-Maandag EC, te Riele HP, ., 1994. Disruption of the mouse mdrla P- 
glycoprotein gene leads to a deficiency in the blood-brain barrier and to increased sensitivity to drugs. Cell 77: 491-502.

Schuetz JD, Silverman JA, Thottassery JV, Furuya KN, Schuetz EG, 1995. Divergent regulation of the class II P-glycoprotein gene in primary cultures of hepatocytes versus H35 hepatoma by glucocorticoids. Cell Growth Differ. 6: 1321-1332.

Shapiro WR, 1985. Animal models: blood-brain barrier and pharmacology. Prog. Exp. Tumor Res. 29: 116-122.

Shapiro WR, Shapiro JR, 1986. Principles of brain tumor chemotherapy. Semin. Oncol. 13: 56-69.

Shapiro WR, Voorhies RM, Hiesiger EM, Sher PB, Basler GA, Lipschutz LE, 1988. Pharmacokinetics of tumor cell exposure to [14C]methotrexate after intracarotid administration without and with hyperosmotic opening of the blood-brain and bloodtumor barriers in rat brain tumors: a quantitative autoradiographic study. Cancer Res. 48: 694-701.

Shen J, Fraga C, Calabrese C, McCarville MB, Schaiquevich P, Stewart CF, 2008. A modified surgical procedure for microdialysis probe implantation in the lateral ventricle of a FVB mouse. J. Pharm. Sci.

Shimada Y, Yoshino M, Wakui A, Nakao I, Futatsuki K, Sakata Y, Kambe M, Taguchi T, Ogawa N, 1993. Phase II study of CPT-11, a new camptothecin derivative, in metastatic colorectal cancer. CPT-11 Gastrointestinal Cancer Study Group. J. Clin. Oncol. 11: 909-913.

Slordal L, Jaeger R, Kjaeve J, Aarbakke J, 1988. Pharmacokinetics of 7-hydroxymethotrexate and methotrexate in the rat. Pharmacol. Toxicol. 63: 81-84.

Smith QR, Johanson CE, Woodbury DM, 1981. Uptake of $36 \mathrm{Cl}$ and $22 \mathrm{Na}$ by the braincerebrospinal fluid system: comparison of the permeability of the blood-brain and bloodcerebrospinal fluid barriers. J. Neurochem. 37: 117-124.

Sparreboom A, Danesi R, Ando Y, Chan J, Figg WD, 2003. Pharmacogenomics of ABC transporters and its role in cancer chemotherapy. Drug Resist. Updat. 6: 71-84.

Sposto R, Ertel IJ, Jenkin RD, Boesel CP, Venes JL, Ortega JA, Evans AE, Wara W, Hammond D, 1989. The effectiveness of chemotherapy for treatment of high grade astrocytoma in children: results of a randomized trial. A report from the Childrens Cancer Study Group. J. Neurooncol. 7: 165-177.

Stahle L, 1994. Zidovudine and alovudine as cross-wise recovery internal standards in microdialysis experiments? J. Pharmacol. Toxicol. Methods 31: 167-169.

Stahle L, Borg N, 2000. Transport of alovudine (3'-fluorothymidine) into the brain and the cerebrospinal fluid of the rat, studied by microdialysis. Life Sci. 66: 1805-1816. 
Stain-Texier F, Boschi G, Sandouk P, Scherrmann JM, 1999. Elevated concentrations of morphine 6-beta-D-glucuronide in brain extracellular fluid despite low blood-brain barrier permeability. Br. J. Pharmacol. 128: 917-924.

Stewart CF, Iacono LC, Chintagumpala M, Kellie SJ, Ashley D, Zamboni WC, Kirstein MN, Fouladi M, Seele LG, Wallace D, Houghton PJ, Gajjar A, 2004a. Results of a phase II upfront window of pharmacokinetically guided topotecan in high-risk medulloblastoma and supratentorial primitive neuroectodermal tumor. J. Clin. Oncol. 22: 3357-3365.

Stewart CF, Leggas M, Schuetz JD, Panetta JC, Cheshire PJ, Peterson J, Daw N, Jenkins JJ, III, Gilbertson R, Germain GS, Harwood FC, Houghton PJ, 2004b. Gefitinib enhances the antitumor activity and oral bioavailability of irinotecan in mice. Cancer Res. 64: 7491-7499.

Stieber VW, Mehta MP, 2007. Advances in radiation therapy for brain tumors. Neurol. Clin. 25: 1005-33, ix.

Straathof CS, van den Bent MJ, Loos WJ, Vecht CJ, Schellens JH, 1999. The accumulation of topotecan in 9L glioma and in brain parenchyma with and without dexamethasone administration. J. Neurooncol. 42: 117-122.

Strazielle N, Ghersi-Egea JF, 2000. Choroid plexus in the central nervous system: biology and physiopathology. J. Neuropathol. Exp. Neurol. 59: 561-574.

Strugar JG, Criscuolo GR, Rothbart D, Harrington WN, 1995. Vascular endothelial growth/permeability factor expression in human glioma specimens: correlation with vasogenic brain edema and tumor-associated cysts. J. Neurosurg. 83: 682-689.

Tait DM, Thornton-Jones H, Bloom HJ, Lemerle J, Morris-Jones P, 1990. Adjuvant chemotherapy for medulloblastoma: the first multi-centre control trial of the International Society of Paediatric Oncology (SIOP I). Eur. J. Cancer 26: 464-469.

Taphoorn MJ, van den Bent MJ, Mauer ME, Coens C, Delattre JY, Brandes AA, Sillevis Smitt PA, Bernsen HJ, Frenay M, Tijssen CC, Lacombe D, Allgeier A, Bottomley A, 2007. Health-related quality of life in patients treated for anaplastic oligodendroglioma with adjuvant chemotherapy: results of a European Organisation for Research and Treatment of Cancer randomized clinical trial. J. Clin. Oncol. 25: 5723-5730.

Thiebaut F, Tsuruo T, Hamada H, Gottesman MM, Pastan I, Willingham MC, 1987. Cellular localization of the multidrug-resistance gene product P-glycoprotein in normal human tissues. Proc. Natl. Acad. Sci. U. S. A 84: 7735-7738.

Tian Q, Zhang J, Chan SY, Tan TM, Duan W, Huang M, Zhu YZ, Chan E, Yu Q, Nie YQ, Ho PC, Li Q, Ng KY, Yang HY, Wei H, Bian JS, Zhou SF, 2006. Topotecan is a substrate for multidrug resistance associated protein 4. Curr. Drug Metab 7: 105-118. 
Tian Q, Zhang J, Tan TM, Chan E, Duan W, Chan SY, Boelsterli UA, Ho PC, Yang H, Bian JS, Huang M, Zhu YZ, Xiong W, Li X, Zhou S, 2005. Human multidrug resistance associated protein 4 confers resistance to camptothecins. Pharm. Res. 22: 1837-1853.

Turner CD, Gururangan S, Eastwood J, Bottom K, Watral M, Beason R, McLendon RE, Friedman AH, Tourt-Uhlig S, Miller LL, Friedman HS, 2002. Phase II study of irinotecan (CPT-11) in children with high-risk malignant brain tumors: the Duke experience. Neuro. -oncol. 4: 102-108.

Ullrich NJ, Pomeroy SL, 2003. Pediatric brain tumors. Neurol. Clin. 21: 897-913.

Ungerstedt U, Pycock C, 1974. Functional correlates of dopamine neurotransmission. Bull. Schweiz. Akad. Med. Wiss. 30: 44-55.

Uziel T, Zindy F, Xie S, Lee Y, Forget A, Magdaleno S, Rehg JE, Calabrese C, Solecki D, Eberhart CG, Sherr SE, Plimmer S, Clifford SC, Hatten ME, McKinnon PJ, Gilbertson RJ, Curran T, Sherr CJ, Roussel MF, 2005. The tumor suppressors Ink4c and p53 collaborate independently with Patched to suppress medulloblastoma formation. Genes Dev. 19: 2656-2667.

Van B, V, Clemente CD, 1955. Silver deposition in the central nervous system and the hematoencephalic barrier studied with the electron microscope. J. Biophys. Biochem. Cytol. 1: 161-166.

Vassal G, Boland I, Santos A, Bissery MC, Terrier-Lacombe MJ, Morizet J, Sainte-Rose C, Lellouch-Tubiana A, Kalifa C, Gouyette A, 1997. Potent therapeutic activity of irinotecan (CPT-11) and its schedule dependency in medulloblastoma xenografts in nude mice. Int. J. Cancer 73: 156-163.

Venkatakrishnan K, Tseng E, Nelson FR, Rollema H, French JL, Kaplan IV, Horner WE, Gibbs MA, 2007. Central nervous system pharmacokinetics of the Mdr1 P-glycoprotein substrate CP-615,003: intersite differences and implications for human receptor occupancy projections from cerebrospinal fluid exposures. Drug Metab Dispos. 35: 13411349.

Vorbrodt AW, Dobrogowska DH, 2003. Molecular anatomy of intercellular junctions in brain endothelial and epithelial barriers: electron microscopist's view. Brain Res. Brain Res. Rev. 42: 221-242.

Wagner S, Erdlenbruch B, Langler A, Gnekow A, Kuhl J, Albani M, Volpel S, Bucsky P, Emser A, Peters O, Wolff JE, 2004. Oral topotecan in children with recurrent or progressive high-grade glioma: a Phase I/II study by the German Society for Pediatric Oncology and Hematology. Cancer 100: 1750-1757.

Wakeling AE, Barker AJ, Davies DH, Brown DS, Green LR, Cartlidge SA, Woodburn JR, 1996. Specific inhibition of epidermal growth factor receptor tyrosine kinase by 4anilinoquinazolines. Breast Cancer Res. Treat. 38: 67-73. 
Wall ME, Wani MC, 1977. Antineoplastic agents from plants. Annu. Rev. Pharmacol. Toxicol. 17: 117-132.

Wang Y, Wong SL, Sawchuk RJ, 1993. Microdialysis calibration using retrodialysis and zero-net flux: application to a study of the distribution of zidovudine to rabbit cerebrospinal fluid and thalamus. Pharm. Res. 10: 1411-1419.

Wei P, Zhang J, Dowhan DH, Han Y, Moore DD, 2002. Specific and overlapping functions of the nuclear hormone receptors CAR and PXR in xenobiotic response. Pharmacogenomics. J. 2: 117-126.

Weindel K, Moringlane JR, Marme D, Weich HA, 1994. Detection and quantification of vascular endothelial growth factor/vascular permeability factor in brain tumor tissue and cyst fluid: the key to angiogenesis? Neurosurgery 35: 439-448.

Wolburg H, Lippoldt A, 2002. Tight junctions of the blood-brain barrier: development, composition and regulation. Vascul. Pharmacol. 38: 323-337.

Wong SL, Van BK, Sawchuk RJ, 1993. Distributional transport kinetics of zidovudine between plasma and brain extracellular fluid/cerebrospinal fluid in the rabbit:

investigation of the inhibitory effect of probenecid utilizing microdialysis. J. Pharmacol. Exp. Ther. 264: 899-909.

Xie R, Hammarlund-Udenaes M, 1998. Blood-brain barrier equilibration of codeine in rats studied with microdialysis. Pharm. Res. 15: 570-575.

Xie R, Hammarlund-Udenaes M, de Boer AG, de Lange EC, 1999. The role of Pglycoprotein in blood-brain barrier transport of morphine: transcortical microdialysis studies in mdrla (-/-) and mdrla (+/+) mice. Br. J. Pharmacol. 128: 563-568.

Yanase K, Tsukahara S, Asada S, Ishikawa E, Imai Y, Sugimoto Y, 2004. Gefitinib reverses breast cancer resistance protein-mediated drug resistance. Mol. Cancer Ther. 3: 1119-1125.

Yang CH, Huang CJ, Yang CS, Chu YC, Cheng AL, Whang-Peng J, Yang PC, 2005. Gefitinib reverses chemotherapy resistance in gefitinib-insensitive multidrug resistant cancer cells expressing ATP-binding cassette family protein. Cancer Res. 65: 6943-6949.

Yang CJ, Horton JK, Cowan KH, Schneider E, 1995. Cross-resistance to camptothecin analogues in a mitoxantrone-resistant human breast carcinoma cell line is not due to DNA topoisomerase I alterations. Cancer Res. 55: 4004-4009.

Young GS, 2007. Advanced MRI of adult brain tumors. Neurol. Clin. 25: 947-73, viii.

Yousif S, Marie-Claire C, Roux F, Scherrmann JM, Decleves X, 2007. Expression of drug transporters at the blood-brain barrier using an optimized isolated rat brain microvessel strategy. Brain Res. 1134: 1-11. 
Zamboni WC, Gajjar AJ, Mandrell TD, Einhaus SL, Danks MK, Rogers WP, Heideman RL, Houghton PJ, Stewart CF, 1998. A four-hour topotecan infusion achieves cytotoxic exposure throughout the neuraxis in the nonhuman primate model: implications for treatment of children with metastatic medulloblastoma. Clin. Cancer Res. 4: 2537-2544.

Zhang C, Beckermann B, Kallifatidis G, Liu Z, Rittgen W, Edler L, Buchler P, Debatin KM, Buchler MW, Friess H, Herr I, 2006. Corticosteroids induce chemotherapy resistance in the majority of tumour cells from bone, brain, breast, cervix, melanoma and neuroblastoma. Int. J. Oncol. 29: 1295-1301.

Zhang Y, Han H, Elmquist WF, Miller DW, 2000. Expression of various multidrug resistance-associated protein (MRP) homologues in brain microvessel endothelial cells. Brain Res. 876: 148-153.

Zheng W, Aschner M, Ghersi-Egea JF, 2003. Brain barrier systems: a new frontier in metal neurotoxicological research. Toxicol. Appl. Pharmacol. 192: 1-11.

Zhuang Y, Fraga CH, Hubbard KE, Hagedorn N, Panetta JC, Waters CM, Stewart CF, 2006. Topotecan central nervous system penetration is altered by a tyrosine kinase inhibitor. Cancer Res. 66: 11305-11313.

Zozulya A, Weidenfeller C, Galla HJ, 2007. Pericyte-endothelial cell interaction increases MMP-9 secretion at the blood-brain barrier in vitro. Brain Res. 


\section{Appendix: Analysis of Topotecan Clearances across BCB or BBB}

The drug movement across the $\mathrm{BBB}$ or $\mathrm{BCB}$ can be generalized as $\mathrm{CL}_{\text {in }}$ (from blood to brain) and $\mathrm{CL}_{\text {out }}$ (from brain to blood) (Hammarlund-Udenaes et al., 1997; Wong et al., 1993; Zhuang et al., 2006). Due to the additive property of clearance, $\mathrm{CL}_{\text {in }}$ or $\mathrm{CL}_{\text {out }}$ can be broken down as follows:

At BCB of FVB mouse

$$
\begin{aligned}
& C L_{\text {in,FVB }}=C L_{B c r p}+C L_{P g p}+C L_{\text {in }, \text { otherATP }}+C L_{\text {in,NONATP }} \\
& C L_{\text {out }, F V B}=C L_{\text {out }, \text { otherATP }}+C L_{\text {out }, \text { NONATP }}
\end{aligned}
$$

At BCB of Bcrpl/Mdrla/b $b^{(--)}$mouse (triple knockout, TKO)

$$
\begin{aligned}
& C L_{\text {in }, T K O}=C L_{\text {in,otherATP }}+C L_{\text {in }, \text { NONATP }} \\
& C L_{\text {out }, T K O}=C L_{\text {out }, \text { otherATP }}+C L_{\text {out }, \text { NONATP }}
\end{aligned}
$$

Subscripts: otherATP-------other than Bcrp1 and P-gp ATP dependent mechanisms

NONATP-------All other ATP independent mediated mechanisms

With gefitinib treatment the AUC ratios were determined 1.15 and 1.1 in FVB and Bcrpl/Mdrla/b $b^{(--)}$mouse models respectively (Figure 3-4), which were statistically the same. According to the relationship of $\mathrm{CL}_{\mathrm{in}} / \mathrm{CL}_{\text {out }}=\mathrm{AUC}_{\mathrm{u}, \mathrm{CSF}} / \mathrm{AUC}_{\mathrm{u} \text {,plasma }}$ (Wong et al., 1993), the following equation can be obtained,

$$
\frac{C L_{\text {in,FVB }}}{C L_{\text {out }, F V B}}=\frac{C L_{\text {in }, T K O}}{C L_{\text {out }, T K O}},
$$

Substitute each item in equation A-5 with equations A-1 to A-4, we obtained

$$
\frac{C L_{B c r p}+C L_{P g p}+C L_{i n, N O N A T P}+C L_{\text {in }, \text { otherATP }}}{C L_{\text {out }, \text { otherATP }}+C L_{\text {out }, \text { NONATP }}}=\frac{C L_{\text {in,NONATP }}+C L_{\text {in,otherATP }}}{C L_{\text {out }, \text { otherATP }}+C L_{\text {out }, \text { NONATP }}}
$$

From equation A-6 and the assumption that gefitinib had the same effect on $\mathrm{CL}_{\text {otherATP }}$ and $\mathrm{CL}_{\text {NONATP }}$ in FVB as in $\mathrm{Bcrp} 1 / \mathrm{Mdrla} / \mathrm{b}^{(--)}$mouse, the following conclusion can be made, with gefitinib treatment, at BCB of FVB mouse

$$
C L_{B c r p}+C L_{P g p}=0
$$

It suggested gefitinib can fully inhibit Bcrp1 and P-gp functions at BCB. 
At BBB of FVB mouse

$$
\begin{aligned}
& C L_{i n, F V B}=C L_{i n, N O N A T P} \\
& C L_{\text {out }, F V B}=C L_{B c r p}+C L_{P g p}+C L_{\text {out }, \text { otherATP }}+C L_{\text {out }, \text { NONATP }}
\end{aligned}
$$

At BBB of Bcrpl/Mdrla/b/(--) mouse (triple knockout, TKO)

$$
\begin{aligned}
& C L_{\text {in }, T K O}=C L_{\text {in,NONATP }} \\
& C L_{\text {out }, T K O}=C L_{\text {out }, \text { otherATP }}+C L_{\text {out }, \text { NONATP }}
\end{aligned}
$$

With gefitinib treatment the AUC ratios were determined 0.63 and 0.95 in FVB and Bcrpl/Mdrla/ $b^{(-)-}$mouse models respectively (Figure 3-4), which were statistically different. The following equations can be obtained,

$$
\begin{aligned}
& \frac{C L_{\text {in, } F V B}}{C L_{\text {out }, F V B}}<\frac{C L_{\text {in }, T K O}}{C L_{\text {out }, T K O}}, \\
& \frac{C L_{\text {in, NONATP }}}{C L_{\text {BCrp }}+C L_{P g p}+C L_{\text {out }, \text { otherATP }}+C L_{\text {out }, \text { NONATP }}}<\frac{C L_{\text {in }, \text { NONATP }}}{C L_{\text {out otherATP }}+C L_{\text {out }, \text { NONATP }}}
\end{aligned}
$$

Then the following conclusion can be made, with gefitinib treatment, at BBB of FVB mouse

$$
C L_{\text {Bcrp }}+C L_{P g p}>0
$$

It suggested gefitinib cannot fully inhibit Bcrp1 and P-gp function at BBB. Taken together, these results indicated gefitinib had differential regulatory effects on $\mathrm{BBB}$ and BCB. Probably it is a more universal phenomenon for many other ABC transporter modulators than just for gefitinib. Thus it warrants the investigation of specific regulatory effects on CNS for the modulators applied to do so even these modulators have shown strong effect on other sites (e.g. gastrointestinal tract) (Kuppens et al., 2007). 


\section{Vita}

Jun Shen was born in China on January $3^{\text {rd }}$, 1973. He received a Bachelor degree in Biochemical Engineering in 1995 from East China University of Science and Technology. After college he worked for Shanghai NLJ Biomedical Co. Ltd., Roche China Ltd., Beijing Norvartis Pharmaceutical Co. Ltd., Shanghai Institute of Physiology and ExxonMobil China Ltd. from 1995 to 2002. He received a Ph.D. degree in the Interdisciplinary Program with a major in Pharmaceutical Sciences from University of Tennessee in 2008. 